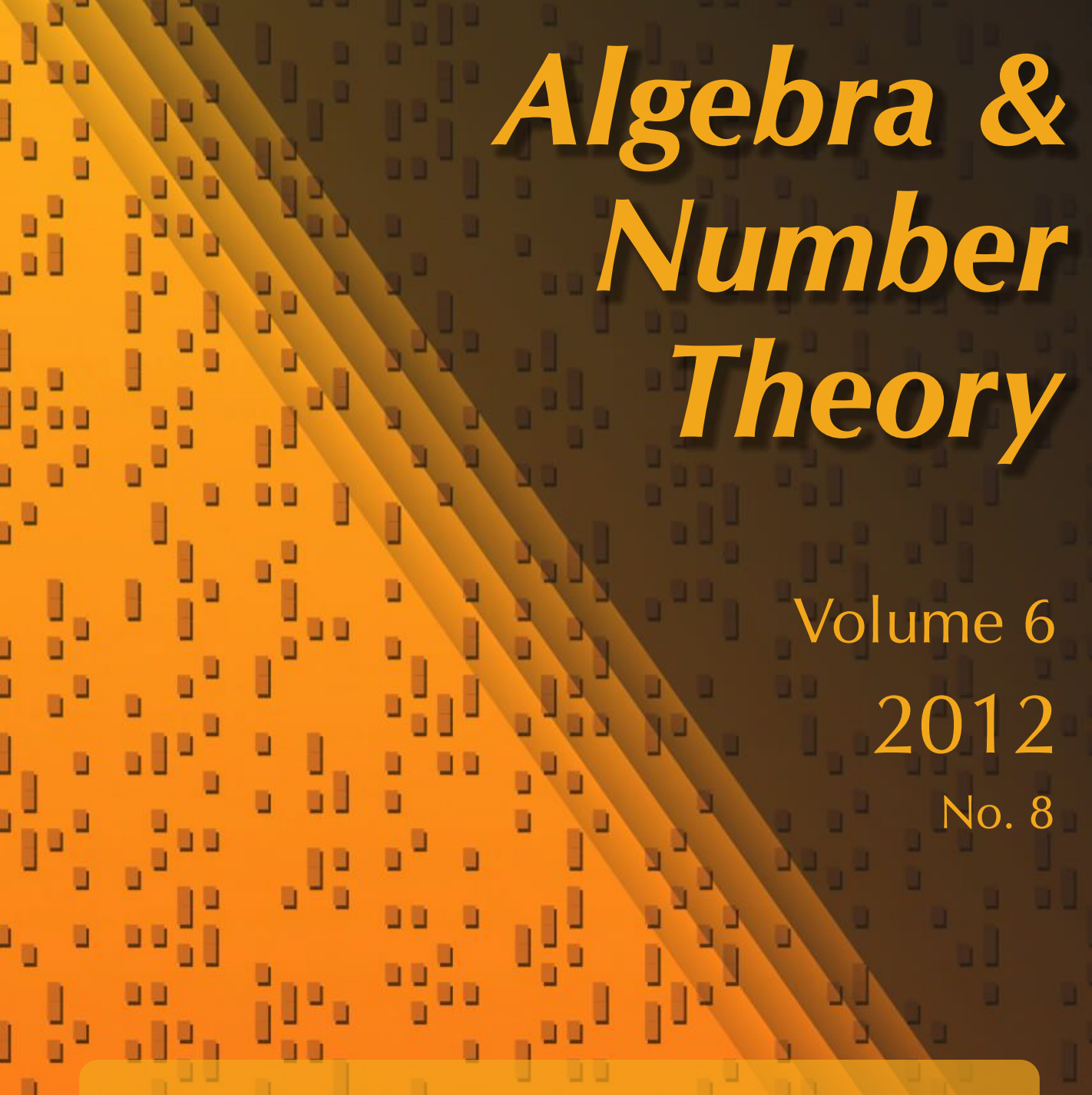

On the refined ramification filtrations in the equal characteristic case

Liang Xiao

\lrcorner$\lrcorner$ 


\title{
On the refined ramification filtrations in the equal characteristic case
}

\author{
Liang Xiao
}

Let $k$ be a complete discrete valuation field of equal characteristic $p>0$. Using the tools of $p$-adic differential modules, we define refined Artin and Swan conductors for a representation of the absolute Galois group $G_{k}$ with finite local monodromy; this leads to a description of the subquotients of the ramification filtration on $G_{k}$. We prove that our definition of the refined Swan conductors coincides with that given by Saito, which uses étale cohomology. We also study its relation with the toroidal variation of Swan conductors.

Introduction

1. Theory of differential modules

2. Refined differential conductors for Galois representations

1623

3. Comparison with Saito's definition

4. Refined Swan conductors and variation of intrinsic radii on polyannuli

\section{Introduction}

The ramification theory for a complete discrete valuation field $k$ with possibly imperfect residue field $\kappa_{k}$ was first studied by K. Kato [1989]; he used étale cohomology and Milnor $K$-theory to give a detailed description of the ramification of a character of the absolute Galois group $G_{k}$, or equivalently of its maximal abelian quotient $G_{k}^{\mathrm{ab}}$. A. Abbes and T. Saito [2002; 2003] extended Kato's work by providing $G_{k}$ with the ramification filtration Fil $^{a} G_{k}$ and the $\log$ ramification filtration $\mathrm{Fil}_{\log }^{a} G_{k}$ satisfying certain properties. Saito [2009] later defined a natural injective homomorphism

$$
\text { rsw }: \operatorname{Hom}\left(\mathrm{Fil}_{\log }^{a} G_{k} / \mathrm{Fil}_{\log }^{a+} G_{k}, \mathbb{F}_{p}\right) \rightarrow \Omega_{\mathscr{O}_{k}}^{1}(\log ) \otimes_{\mathscr{O}_{k}} \pi_{k}^{-a} \kappa_{k}^{\text {alg }}
$$

MSC2000: primary 11S15; secondary 14G22, 11S80, 11S31.

Keywords: ramification filtration, Swan conductor, refined Swan conductor, p-adic differential module, Dwork isocrystal. 
for each $a \in \mathbb{Q}_{>0}$, where $\mathcal{O}_{k}$ is the ring of integers of $k, \pi_{k}$ is a uniformizer, $\kappa_{k}$ is the residue field, and $\Omega_{k}^{1}(\log )$ is the module of logarithmic differentials; he called it the refined Swan conductor homomorphism. This provides some further information about the subquotients for the $\log$ ramification filtration on $G_{k}$.

Along a different path, G. Christol, B. Dwork, S. Matsuda, Z. Mebhkout, and their collaborators used $p$-adic differential modules to give an interpretation of the Swan conductors of representations of $G_{k}$ when the residue field $\kappa_{k}$ is perfect. They associated a $p$-adic differential module over an annulus to any continuous representation of $G_{k}$, and proved that the Swan conductor of the representation is related to the radii of convergence of the local solutions for the differential module. K. Kedlaya [2007] generalized this approach to include the case in which the residue field is imperfect, by giving the definitions of Artin conductors and Swan conductors for a representation of $G_{k}$. The author [Xiao 2010] verified that this pair of definitions coincide with those naturally associated to the ramification filtration and $\log$ ramification filtration of Abbes and Saito [2002; 2003]. An important consequence of this comparison result is the Hasse-Arf theorem for the ramification filtration and the log one [Xiao 2010, Theorem 4.4.1], which states that the Artin conductors and Swan conductors are all integers.

In this paper, we give an alternative definition of the refined Swan conductor homomorphism as well as their nonlog counterparts, using $p$-adic differential modules, and we will compare our definition with that of Saito. Let us describe the basic idea of the definition. In this introduction, we assume for simplicity that $\kappa_{k}$ has a finite $p$-basis $\left\{\bar{b}_{1}, \ldots, \bar{b}_{m}\right\}$. Let $K$ be the fraction field of the Cohen ring of $\kappa_{k}$ with respect to $\bar{b}_{1}, \ldots, \bar{b}_{m}$. Let $B_{1}, \ldots, B_{m}$ denote the canonical lifts of $\bar{b}_{1}, \ldots, \bar{b}_{m}$ to $K$, respectively. Let $A_{K}^{1}\left(\eta_{0}, 1\right)$ be the annulus over $K$ with coordinate $T$ and with radii in $\left(\eta_{0}, 1\right)$ for some $\eta_{0} \in(0,1)$. By the aforementioned series of work, one can associate to an irreducible $p$-adic representation $\rho$ of $G_{k}$ with finite image a differential module $\mathscr{E}$ over $A_{K}^{1}\left(\eta_{0}, 1\right)$ for the differential operators $\partial_{0}=\partial / \partial T$ and $\partial_{1}=\partial / \partial B_{1}, \ldots, \partial_{m}=\partial / \partial B_{m}$. Let $\pi=-p^{1 /(p-1)}$ denote a Dwork pi and put $K^{\prime}=K(\pi)$. When $\rho$ is of pure ramification break $b$, that is, when $\rho\left(\mathrm{Fil}^{b+} G_{k}\right)$ is trivial but $\rho\left(\mathrm{Fil}^{b} G_{k}\right)$ is not, the following naïve picture is helpful as a guide to intuition. Suppose that there exists a basis of $\mathscr{E} \otimes_{K} K^{\prime}$, with respect to which $\partial_{0}, \partial_{1}, \ldots, \partial_{m}$ act per the prescription:

$$
\partial_{0}=\pi T^{-b-1} N_{0}, \quad \partial_{1}=\pi T^{-b} N_{1}, \ldots, \partial_{m}=\pi T^{-b} N_{m},
$$

where $N_{0}, \ldots, N_{m}$ are matrices in $\mathcal{O}_{K^{\prime}} \llbracket T \rrbracket$. For each $j \in\{0, \ldots, m\}$, we use $\bar{N}_{j}$ to denote reduction of $N_{j}$ modulo the ideal $(\pi, T)$; these matrices commute and have coefficients in $\kappa_{k}$. Take a common (generalized) eigenbasis $e_{1}, \ldots, e_{d}$ for all $\bar{N}_{j}$; set $\theta_{i, j}$ to be the (generalized) eigenvalue of $\bar{N}_{j}$ associated to $e_{i}$, viewed as an element in $\kappa_{k}^{\text {alg }}$. One may then define the multiset of refined Swan conductors of $\rho$ 
to be

$$
\left\{\pi_{k}^{-b}\left(\theta_{i, 0} \frac{d \pi_{k}}{\pi_{k}}+\theta_{i, 1} d \bar{b}_{1}+\cdots+\theta_{i, m} d \bar{b}_{m}\right): i=1, \ldots, d\right\} \subset \Omega_{\bigcirc_{k}}^{1}(\log ) \otimes_{\bigcirc_{k}} \pi_{k}^{-b} \kappa_{k}^{\text {alg }} .
$$

(A multiset is a set where we allow elements to have multiplicity.) Of course, such a nice basis of $\mathscr{E} \otimes_{K} K^{\prime}$ over the annulus $A_{K^{\prime}}^{1}\left[\eta_{0}, 1\right)$ with the described properties might not exist in general. In practice, we need the following two technical arguments to read off the multiset of refined Swan conductors.

(a) The above picture can be better described over a field. Namely, we have the description of the actions of $\partial_{0}, \ldots, \partial_{m}$ as in (0.0.1) over the completion of $K(T)$ with respect to the $\eta$-Gauss norm for any $\eta \in\left[\eta_{0}, 1\right)$. By taking common eigenvalues as explained above, we can define a version of refined Swan conductors, called the refined radii, of the differential module at each radius $\eta$. We then show that the refined radii, as we vary the radius of the Gauss norm, also vary in a nice way when $\eta$ is sufficiently close to 1 : they form a unique multiset consisting of elements of $\Omega_{\mathscr{O}_{k}}^{1}(\log ) \otimes_{\mathscr{O}_{k}} \pi_{k}^{-b} \kappa_{k}^{\text {alg }}$, independent of the choice of $\eta$. We then just simply define this multiset to be the multiset of refined Swan conductors of the representation $\rho$; this does not require any good matrices representing the actions of $\partial_{j}$ over the entire annulus.

(b) When the spectral norms of the differential operators are smaller than their operator norms over the base field, the description (0.0.1) requires some modification. Over the completion of $K(T)$ for the $\eta$-Gauss norm, we may find a basis such that the matrix for $\partial_{j}^{p^{r}}$ with an appropriate $r \in \mathbb{N}$ acts by some nice matrix as in (0.0.1). We then take the common eigenvalues of those matrices and define the refined radii to be the $p^{r}$-th roots of these eigenvalues. When trying to prove results in this case, we use a technique called Frobenius antecedents developed in [Kedlaya and Xiao 2010], which reduces the question at hand to the case when the spectral norms are bigger than the operator norms.

We can also define the notion of refined Artin conductors using a variant of the definition of the refined Swan conductors, in which the effect of log structure is removed, which amounts to replacing the factor $T^{-b-1}$ by $T^{-b}$ in (0.0.1).

Part of the content in this paper on refined Swan conductors has been already included in the author's thesis [Xiao 2009]. However, we feel the present paper provides a better context for our development of refined Swan conductors. We also fill in some gaps in the thesis.

To compare our definition of refined Swan conductors with Saito's, we proceed as in [Xiao 2010] by introducing the thickening spaces which tie the $p$-adic differential equations together with the rigid analytic spaces considered by Abbes and Saito. More precisely, we may first realize a finite Galois extension $l$ of $k$ as the corresponding extension of the function fields of a finite étale extension of 
smooth affine varieties $Y \rightarrow X$. We may further assume that both $X$ and $Y$ lift to smooth formal schemes $\boldsymbol{X}$ and $\boldsymbol{Y}$. The differential module associated to a $p$-adic representation of $\mathrm{Gal}(l / k)$ lives over the a subspace of the tube of $X$ embbeded diagonally in $\boldsymbol{X} \times \boldsymbol{X}$, which is a rigid analytic subspace of the generic fiber of $\boldsymbol{X} \times \boldsymbol{X}$ and is called the thickening space. We carefully study the construction of the differential module and compare that with Saito's description of the special fiber of the formal scheme $\boldsymbol{Y}$. The core of the comparison result is to identify the data defining an Artin-Schreier cover of $\mathbb{A}_{\kappa_{k}}^{m}$ with the data coming from the associated Dwork isocrystals as a differential module.

We also remark that when $k$ is an $n$-dimensional higher local field of characteristic $p>0$, the refined conductors induce a ramification filtration on $G_{k}$ indexed by $\mathbb{Q}^{n}$ with lexicographic order. This is expected to be compatible with certain filtration on the Milnor $K$-groups via Kato's class field theory.

Finally, we study the relation of the refined Swan conductors with the variation of intrinsic radii (certain form of Swan conductors) over a polyannulus. We prove that the valuations of the refined Swan conductors at a vertex of the polygon associated to the polyannulus encode some information about the slopes of the log-affine functions of the intrinsic radii at that vertex. For the precise statement, we refer to Proposition 4.3.13.

Plan of the paper. Section 1 is devoted to developing the theory of refined radii, the analog of refined conductors over a complete nonarchimedean field. In the first two subsections, we set up notation and recall some basic results on differential modules from [Kedlaya and Xiao 2010]. We define the refined radii in Section 1.3 and prove a decomposition result (Theorem 1.3.26) that separates pieces with different refined radii in a differential module. In Section 1.4 we consider the case where we allow multiple derivations to interact. In Section 1.5 we study how the refined radii vary on an annulus or a disc, when the radii are log-affine functions. We then define the refined conductors for solvable differential modules over an annulus in Section 1.6.

In Section 2 we apply the theory of refined conductors for solvable differential modules to define refined conductors for Galois representations. In the first two subsections we recall the construction of differential modules following [Kedlaya 2007], and deduce some basic properties. In Section 2.3 we define the refined conductor homomorphism. Section 2.4 briefly discusses an application to higher local fields.

In Section 3 we compare our definition with that of Saito, which is reviewed in Section 3.1. In Section 3.2 we realize the extension of fields as a finite étale cover of varieties and lift them to rigid analytic spaces over $K$. In Section 3.3 we do a crucial calculation on the differential module structure of Dwork isocrystals to determine their refined radii; this calculation forms the heart of our proof of 
the comparison theorem. We wrap up Section 3 with a proof of the comparison Theorem 3.4.1 in Section 3.4.

In Section 4 we focus on the interplay of refined Swan conductors with the toroidal variation of Swan conductors. A few technical lemmas are discussed in Section 4.2, and the main theorems are proved in Section 4.3.

\section{Theory of differential modules}

Our systematic study of differential modules proceeds in two stages: first over a complete nonarchimedean field, and then over an annulus over a complete nonarchimedean field. In the former case, the spectral norm, or equivalently the radius of convergence, of the differential operator is a very important invariant; when the differential module has pure radii, we will focus on certain secondary information of the differential module, called the refined radii. In the latter case, it was proved in [Kedlaya and Xiao 2010] that the radii of convergence of a differential module over an annulus give rise to piecewise log-affine functions as one varies the radii on the annulus; we will again focus on the secondary data: the refined radii. In the case when the aforementioned piecewise log-affine functions are in fact log-affine, we prove that the multisets of refined radii of the differential module at all radii are the same, if we naturally identify the spaces where these refined radii live.

1.1. Setup. This subsection is mainly to explain our convention on notations; however, the commutative algebra Lemma 1.1 .10 will become a very useful tool later as explained in Remark 1.1.11.

Notation 1.1.1. By a multiset $S$, we mean a set where we allow elements to have multiplicity. For $s \in S$, the multiplicity of $s$ in $S$ is denoted by $\operatorname{multi}_{S}(S)$. When $S$ consists of a single element (with multiplicity), we call it pure.

Notation 1.1.2. For any field $K$ that will be considered in this paper, $K^{\text {alg }}$ will denote a fixed algebraic closure. We let $K^{\text {sep }}$ denote the separable closure of $K$ inside $K^{\text {alg }}$. Set $G_{K}=\operatorname{Gal}\left(K^{\text {sep }} / K\right)$. For a finite Galois extension $L / K$ (inside $\left.K^{\text {sep }}\right)$, we denote its Galois group by $G_{L / K}=\mathrm{Gal}(L / K)$.

For $e \in \mathbb{N}$, we use $\mu_{e}$ to denote the set of $e$-th roots of unity in $K^{\text {alg }}$.

Notation 1.1.3. By a nonarchimedean field, we mean a field $K$ equipped with a nonarchimedean norm $|\cdot|=|\cdot|_{K}: K^{\times} \rightarrow \mathbb{R}_{+}^{\times}$. A subring of $K$ (with the induced norm and topology) is called a nonarchimedean ring.

For a nonarchimedean field $K$, denote the ring of integers of $K$ by

$$
\mathrm{O}_{K}=\{x \in K:|x| \leq 1\}
$$

and the maximal ideal of $\mathscr{O}_{K}$ by $\mathfrak{m}_{K}=\{x \in K:|x|<1\}$; denote the residue field of $K$ by $\kappa_{K}=\mathfrak{O}_{K} / \mathfrak{m}_{K}$. We reserve the letter $p$ for the characteristic of $\kappa_{K}$. If 
char $\kappa_{K}=p>0$ and char $K=0$, we normalize the norm on $K$ so that $|p|=1 / p$. For an element $a \in \mathcal{O}_{K}$, we denote its image in $\kappa_{K}$ under the reduction map by $\bar{a}$. In case $K$ is discretely valued, let $\pi_{K}$ denote a uniformizer of $O_{K}$ and let $v_{K}(\cdot)$ be the corresponding valuation on $K$, normalized so that $v_{K}\left(\pi_{K}\right)=1$.

For a nonarchimedean field $K$ and $s \in \mathbb{R}$, we set

$$
\mathfrak{m}_{K}^{(s)}=\left\{x \in K:|x| \leq \mathrm{e}^{-s}\right\}, \quad \mathfrak{m}_{K}^{(s)+}=\left\{x \in K:|x|<\mathrm{e}^{-s}\right\}, \quad \kappa_{K}^{(s)}=\mathfrak{m}_{K}^{(s)} / \mathfrak{m}_{K}^{(s)+} .
$$

If $s \in-\log \left|K^{\times}\right|$, there exists a noncanonical isomorphism $\kappa_{K} \simeq \kappa_{K}^{(s)}$. For $a \in K$ with $|a| \leq \mathrm{e}^{-s}$, we sometimes denote its image in $\kappa_{K}^{(s)}$ by $\bar{a}^{(s)}$. In particular, $\kappa_{K}^{(0)}=\kappa_{K}$ and $\bar{a}^{(0)}=\bar{a}$ if $v(a) \geq 0$.

Notation 1.1.4. Let $J$ be an index set. We use $e_{J}$ to denote a tuple $\left(e_{j}\right)_{j \in J}$. For another tuple $u_{J}$, set $u_{J}^{e_{J}}=\prod_{j \in J} u_{j}^{e_{j}}$, if all but finitely many of the $e_{j}$ are equal to 0 . We also use $\sum_{e_{J}=0}^{n}$ to denote the sum over $e_{j} \in\{0,1, \ldots, n\}$ for each $j \in J$ provided $e_{j} \neq 0$ for only finitely many $j$; for notational simplicity, we may suppress the range of the summation when it is clear. If $J$ is finite, put

$$
\left|e_{J}\right|=\sum_{j \in J}\left|e_{j}\right| \quad \text { and } \quad\left(e_{J}\right) !=\prod_{j \in J}\left(e_{j} !\right) .
$$

Convention 1.1.5. Throughout this paper, all derivations on topological modules will be assumed to be continuous; in particular, $\Omega_{R / S}^{1}$ will denote the module of continuous differentials on the (topological) ring $R$ relative to the (topological) base ring $S$; we may suppress $S$ from the notation when $S=\mathbb{F}_{p}, \mathbb{Z}$ or $\mathbb{Z}_{p}$. Moreover, all derivations on nonarchimedean rings will be assumed to be bounded (that is, to have bounded operator norms). All connections considered will be assumed to be integrable.

Notation 1.1.6. For a matrix $A=\left(A_{i j}\right)$ with coefficients in a nonarchimedean ring, we use $|A|$ to denote the supremum among the norms of the entries $A_{i j}$ of $A$.

Hypothesis 1.1.7. For the rest of this subsection, we assume that $K$ is a complete nonarchimedean field.

Notation 1.1.8. Let $I \subset[0,+\infty)$ be an interval and let $n \in \mathbb{N}$. Let

$$
A_{K}^{n}(I)=\left\{\left(x_{1}, \ldots, x_{n}\right) \in K^{\text {alg }}:\left|x_{i}\right| \in I \text { for } i=1, \ldots, n\right\}
$$

denote the polyannulus of dimension $n$ with radii in $I$. (We do not impose any rationality condition on the endpoints of $I$, so this space should be viewed as an analytic space in the sense of Berkovich [1990].) If $I$ is written explicitly in terms of its endpoints (e.g., $[\alpha, \beta]$ ), we suppress the parentheses around $I$ (e.g., $A_{K}^{n}[\alpha, \beta]$ ). 
Notation 1.1.9. Let $0<\alpha \leq \beta<+\infty$. We put

$$
\begin{aligned}
& K\langle\alpha / t, t / \beta\rangle=\left\{\sum_{n \in \mathbb{Z}} a_{n} t^{n}:\left|a_{n}\right| \eta^{n} \rightarrow 0 \text { as } n \rightarrow \pm \infty, \text { for any } \eta \in[\alpha, \beta]\right\}, \\
& K\langle\alpha / t, t / \beta\}\}=\left\{\sum_{n \in \mathbb{Z}} a_{n} t^{n}:\left|a_{n}\right| \eta^{n} \rightarrow 0 \text { as } n \rightarrow \pm \infty, \text { for any } \eta \in[\alpha, \beta)\right\}, \\
& K\left\{\left\{\alpha / t, t / \beta \rrbracket_{0}=\left\{\sum_{n \in \mathbb{Z}} a_{n} t^{n}:\left|a_{n}\right| \eta^{n} \rightarrow 0 \text { and }\left|a_{n}\right| \beta^{n}\right. \text { is bounded }\right.\right. \\
& \text { as } n \rightarrow \pm \infty \text {, for any } \eta \in(\alpha, \beta)\} \text {. } \\
& K\langle t / \beta\rangle=\left\{\sum_{n=0}^{\infty} a_{n} t^{n}:\left|a_{n}\right| \beta^{n} \rightarrow 0 \text { as } n \rightarrow+\infty\right\}, \\
& K\{\{t / \beta\}\}=\left\{\sum_{n=0}^{\infty} a_{n} t^{n}:\left|a_{n}\right| \eta^{n} \rightarrow 0 \text { as } n \rightarrow+\infty \text {, for any } \eta \in[0, \beta)\right\}, \\
& K \llbracket t / \beta \rrbracket_{0}=\left\{\sum_{n=0}^{\infty} a_{n} t^{n}:\left|a_{n}\right| \beta^{n} \text { is bounded as } n \rightarrow \infty\right\} .
\end{aligned}
$$

For $I=\{1, \ldots, n\}$ and a nonarchimedean ring $R$, we use $R\left\langle u_{I}\right\rangle$ to denote the Tate algebra, consisting of formal power series $\sum_{e_{I} \geq 0} a_{e_{I}} u_{I}^{e_{I}}$ with $a_{e_{I}} \in R$ and $\left|a_{e_{I}}\right| \rightarrow 0$ as $\left|e_{I}\right| \rightarrow+\infty$. For $\left(\eta_{i}\right)_{i \in I} \in(0,+\infty)^{n}$, the $\eta_{I}$-Gauss norm on the polynomial ring $R\left[t_{I}\right]$ is the norm $|\cdot|_{\eta_{I}}$ given by

$$
\left|\sum_{e_{I}} a_{e_{I}} t_{I}^{e_{I}}\right|_{\eta_{I}}=\max _{e_{I}}\left\{\left|a_{e_{I}}\right| \cdot \eta_{I}^{e_{I}}\right\} ;
$$

this norm extends uniquely to multiplicative norms on $\operatorname{Frac}\left(R\left[t_{I}\right]\right)$, and on $R\left\langle t_{I}\right\rangle$ in case $\left|\eta_{i}\right| \leq 1$ for any $i \in I$.

For $\eta \in[\alpha, \beta]$, the $\eta$-Gauss norm on $K[t]$ extends to multiplicative norms on $K\langle\alpha / t, t / \beta\rangle$ and $K \llbracket t / \beta \rrbracket_{0}$, on $\left.K\langle\alpha / t, t / \beta\}\right\}$ in case $\eta \neq \beta$, and on $K\left\{\left\{\alpha / t, t / \beta \rrbracket_{0}\right.\right.$ in case $\eta \neq \alpha$.

We record here a lemma in commutative algebra which will be frequently used (implicitly) when gluing decompositions.

Lemma 1.1.10. Let

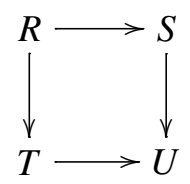

be a commuting diagram of inclusions of integral domains, such that the intersection $S \cap T$ within $U$ is equal to $R$. Let $M$ be a finite locally free $R$-module. Then the intersection of $M \otimes_{R} S$ and $M \otimes_{R} T$ within $M \otimes_{R} U$ is equal to $M$.

Proof. See [Kedlaya and Xiao 2010, Lemma 2.3.1]. 
Remark 1.1.11. We explain how this lemma is used in this paper. We often apply this lemma to the $R$-module $\operatorname{End}(M)$ over $R$ for a differential module $M$. More precisely, we often encounter the situation when we can write both $M \otimes_{R} S$ and $M \otimes_{R} T$ as direct sums of two submodules such that both direct sum decompositions, when tensored with $U$, give the same direct sum decomposition of $M \otimes_{R} U$. We view the projections constituting the direct sum decompositions as elements in $\operatorname{End}(M) \otimes_{R} S, \operatorname{End}(M) \otimes_{R} T$, and $\operatorname{End}(M) \otimes_{R} U$, respectively. By Lemma 1.1.10, we see that the projections above are actually the images of one element of $\operatorname{End}(M)$ under the natural maps; this element defines a direct sum decomposition of $M$ which when tensored with $S$ or $T$ yields the given direct sum decomposition of $M \otimes_{R} S$ or $M \otimes_{R} T$, respectively. In other words, we can "glue" the direct sum decompositions of $M \otimes_{R} S$ and of $M \otimes_{R} T$ along $M \otimes_{R} U$ to get a direct sum decomposition of $M$ (over $R$ ).

1.2. Differential modules and radii of convergence. The starting point of the theory of nonarchimedean differential modules is to understand differential modules over a nonarchimedean field. One of the important tools is the Newton polygon associated to a cyclic vector, which gives much numerical information if the spectral norm of the differential operator is strictly bigger than the operator norm on the base field. To extend interesting results across the threshold imposed by the operator norm mentioned above, we restrict ourselves to the case when the differential operator is of rational type, that is, its metric properties resemble $d / d X$ acting on the completion of $\mathbb{Q}_{p}(X)$ with respect to the 1-Gauss norm; in this case, we may entirely remove the restriction on spectral norms by considering the Frobenius antecedents of the differential modules.

Definition 1.2.1. Let $K$ be a differential ring, that is, a ring equipped with a derivation $\partial$. Let $K\{T\}$ denote the (noncommutative) ring of twisted polynomials over $K$ [Ore 1933]; its elements are finite formal sums $\sum_{i \geq 0} a_{i} T^{i}$ with $a_{i} \in K$, multiplied according to the rule $T a=a T+\partial(a)$ for $a \in K$.

A $\partial$-differential module over $K$ is a finite projective $K$-module $V$ equipped with an action of $\partial$ (subject to the Leibniz rule); any $\partial$-differential module over $K$ inherits a left action of $K\{T\}$ where $T$ acts via $\partial$. The rank of $V$ is the rank of $V$ as a $K$-module. The module dual $V^{\vee}=\operatorname{Hom}_{K}(V, K)$ of $V$ may be viewed as a $\partial$-differential module by setting $(\partial f)(\boldsymbol{v})=\partial(f(\boldsymbol{v}))-f(\partial(\boldsymbol{v}))$. We say $V$ is free if $V$ is free as a module over $K$. We say $V$ is trivial if it is isomorphic to $K^{\oplus d}$ for some $d \in \mathbb{N}$ as a $\partial$-differential module.

For a $\partial$-differential module $V$ free of rank $d$ over $K$, an element $\boldsymbol{v} \in V$ is called a cyclic vector if $\boldsymbol{v}, \partial \boldsymbol{v}, \ldots, \partial^{d-1} \boldsymbol{v}$ form a basis of $V$ as a $K$-module. A cyclic vector defines an isomorphism $V \simeq K\{T\} / K\{T\} P$ of $\partial$-differential modules, where $P \in K\{T\}$ is some monic twisted polynomial of degree $d$, and the $\partial$-action on 
$K\{T\} / K\{T\} P$ is the left multiplication by $T$. If $K$ is a differential field of characteristic 0, $V$ always has a cyclic vector; see [Dwork et al. 1994, Theorem III.4.2; Kedlaya 2010, Theorem 5.4.2].

For a $\partial$-differential module $V$, we put $H_{\partial}^{0}(V)=\operatorname{Ker} \partial$.

Hypothesis 1.2.2. For the rest of this subsection, we assume that $K$ is a complete nonarchimedean field of characteristic zero, equipped with a derivation $\partial$ with operator norm $|\partial|_{K}<\infty$, and that $V$ is a nonzero $\partial$-differential module over $K$.

Definition 1.2.3. Let $p$ denote the residual characteristic of $K$; we conventionally set

$$
\omega= \begin{cases}1 & \text { if } p=0 \\ p^{-1 /(p-1)} & \text { if } p>0\end{cases}
$$

The spectral norm of $\partial$ on $V$ is defined to be $|\partial|_{\mathrm{sp}, V}=\lim _{n \rightarrow \infty}\left|\partial^{n}\right|_{V}^{1 / n}$ for any fixed $K$-compatible norm $|\cdot|_{V}$ on $V$. Define the generic $\partial$-radius of $V$ to be $R_{\partial}(V)=\omega|\partial|_{\mathrm{sp}, V}^{-1}$; note that $R_{\partial}(V)>0$. Let $V_{1}, \ldots, V_{d}$ be the Jordan-Hölder constituents of $V$ as a $K\{T\}$-module. We define the multiset $\Re_{\partial}(V)$ of (extrinsic) subsidiary d-radii of $V$ to be the collection of $R_{\partial}\left(V_{i}\right)$ with multiplicity $\operatorname{dim} V_{i}$ for $i=1, \ldots, d$. Let $R_{\partial}(V ; 1) \leq \cdots \leq R_{\partial}(V ; \operatorname{dim} V)$ denote the elements of $\Re_{\partial}(V)$ in nondecreasing order. We say that $V$ has pure $\partial$-radii if $\Re_{\partial}(V)$ is pure as a multiset; in other words, it consists of $\operatorname{dim} V$ copies of $R_{\partial}(V)$.

Definition 1.2.4. Let $R$ be a complete $K$-algebra. For $v \in V$ and $x \in R$, we define the $\partial$-Taylor series of $\boldsymbol{v}$ with respect to $x$ to be

$$
\mathbb{T}(\boldsymbol{v} ; \partial ; x)=\sum_{n=0}^{\infty} \frac{\partial^{n}(\boldsymbol{v})}{n !} x^{n} \in V \otimes_{K} R,
$$

in case this series converges. When $V=K$, the $\partial$-Taylor series (1.2.5) with respect to a fixed $x \in R$ gives a homomorphism $K \rightarrow R$ of rings, if it converges for all $v \in V=K$. For general $V$, the $\partial$-Taylor series (1.2.5) with respect to the same fixed $x \in R$ gives a homomorphism of $K$-modules $V \rightarrow V \otimes_{K} R$ respecting the aforementioned ring homomorphism, if both homomorphisms converge.

Lemma 1.2.6. Let $V, V_{1}$, and $V_{2}$ be nonzero $\partial$-differential modules over $K$.

(a) If $0 \rightarrow V_{1} \rightarrow V \rightarrow V_{2} \rightarrow 0$ is exact, then we have $\mathfrak{R}_{\partial}(V)=\mathfrak{R}_{\partial}\left(V_{1}\right) \cup \mathfrak{R}_{\partial}\left(V_{2}\right)$.

(b) We have $\mathfrak{R}_{\partial}\left(V^{\vee}\right)=\mathfrak{R}_{\partial}(V)$.

(c) We have $R_{\partial}\left(V_{1} \otimes V_{2}\right) \geq \min \left\{R_{\partial}\left(V_{1}\right), R_{\partial}\left(V_{2}\right)\right\}$. If $V_{1}$ is irreducible and $R_{\partial}\left(V_{1}\right)<R_{\partial}\left(V_{2}\right)$, then $V_{1} \otimes V_{2}$ has pure $\partial$-radius $R_{\partial}\left(V_{1}\right)$.

(d) Let $f: K \rightarrow K \llbracket T / u \rrbracket_{0}$ be the homomorphism given by $f(x)=\mathbb{T}(x ; \partial ; T)$. Then $f^{*} V=V \otimes_{K, f} K \llbracket T / u \rrbracket_{0}$ is a $\partial_{T}=\partial / \partial T$-differential module over 
$K \llbracket T / u \rrbracket_{0}$. For $r \in\left(0, R_{\partial}(K)\right), R_{\partial}(V) \geq r$ if and only if $f^{*} V$ restricts to a trivial $\partial_{T}$-differential module over $A_{K}^{1}[0, r)$.

Proof. The statements (a)-(c) are [Kedlaya and Xiao 2010, Lemma 1.2.9] and the statement (d) is [ibid., Proposition 1.2.14].

Definition 1.2.7. For $P(T)=\sum_{i} a_{i} T^{i} \in K[T]$ or $K\{T\}$ a nonzero (possibly twisted) polynomial, define the Newton polygon of $P$ as the lower convex hull of the set $\left\{\left(-i,-\log \left|a_{i}\right|\right)\right\} \subset \mathbb{R}^{2}$.

Proposition 1.2.8 (Christol-Dwork). Suppose that $V \simeq K\{T\} / K\{T\} P$, and let $s$ be the lesser of $-\log |\partial|_{K}$ and the least slope of the Newton polygon of $P$. Then $\max \left\{|\partial|_{K},|\partial|_{\mathrm{sp}, V}\right\}=\mathrm{e}^{-s}$. More generally, the multiplicity of any $s^{\prime}<-\log |\partial|_{K}$ as a slope of the Newton polygon of $P$ coincides with the multiplicity of $\omega \mathrm{e}^{s^{\prime}}$ in $\Re_{\partial}(V)$. Proof. This is [Kedlaya 2010, Theorem 6.5.3].

Definition 1.2.9. We say a derivation $\partial$ on $K$ is of rational type if there exists $u \in K$ such that the following conditions hold (in this case, we call $u$ a rational parameter for $\partial$ ):

(i) we have $\partial(u)=1$ and $|\partial|_{K}=|u|^{-1}$, and

(ii) for each positive integer $n,\left|\partial^{n} / n !\right|_{K} \leq|\partial|_{K}^{n}$.

If $\partial$ is of rational type, the inequalities in (ii) are in fact equalities, which yields that $|\partial|_{\mathrm{sp}, K}=\omega|\partial|_{K}$; see [Kedlaya and Xiao 2010, Definition 1.4.1].

Lemma 1.2.10. Let $\partial$ be a derivation on $K$ of rational type with $u$ as a rational parameter and let $L / K$ be a finite tamely ramified extension. Then the unique extension of $\partial$ to $L$ is of rational type with $u$ again as a rational parameter.

Proof. This is [Kedlaya and Xiao 2010, Lemma 1.4.5].

Remark 1.2.11. We sometimes need to replace $K$ by the completion of $K(x)$ with respect to the $\eta$-Gauss norm for some $\eta \in \mathbb{R}_{>0}$, where $x$ is transcendental over $K$ and we set $\partial x=0$. The derivation $\partial$ is again of rational type when acting on the new field.

Definition 1.2.12. When $\partial$ is of rational type, it is more convenient to consider $\partial$-radii with a different normalization, as follows. For $V$ a $\partial$-differential module, we define the intrinsic $\partial$-radius of $V$ to be $I R_{\partial}(V)=|\partial|_{\mathrm{sp}, K} /|\partial|_{\mathrm{sp}, V}=|\partial|_{K} \cdot R_{\partial}(V)$. We define the multiset of intrinsic subsidiary $\partial$-radii to be $\mathfrak{I} \mathfrak{R}_{\partial}(V)=|\partial|_{K} \cdot \mathfrak{R}_{\partial}(V)$. We put $I R_{\partial}(V ; i)=|\partial|_{K} \cdot R_{\partial}(V ; i)$ for $i=1, \ldots, \operatorname{dim} V$. We say that $V$ has pure intrinsic $\partial$-radii if $\mathfrak{I}_{\partial}(V)$ is pure as a multiset.

Hypothesis 1.2.13. For the rest of this subsection, we assume that $K$ is a complete nonarchimedean field of characteristic zero and residual characteristic $p$, equipped with a derivation $\partial$ of rational type. We $f i x u \in K$ a rational parameter of $\partial$. We also assume $p>0$ unless otherwise specified. 
Construction 1.2.14. We construct the $\partial$-Frobenius as follows. If $K$ contains a primitive $p$-th root of unity $\zeta_{p}$, we may define an isometric action of the group $\mathbb{Z} / p \mathbb{Z}$ on $K$ using $\partial$-Taylor series:

$$
x^{(i)}=\mathbb{T}\left(x ; \partial ;\left(\zeta_{p}^{i}-1\right) u\right) \quad(i \in \mathbb{Z} / p \mathbb{Z}, x \in K) ;
$$

in particular, $u^{(i)}=\zeta_{p}^{i} u$. Let $K^{(\partial)}$ be the fixed subfield of $K$ under this action; in particular, $u^{p} \in K^{(\partial)}$. Hence, we have a Galois extension $K / K^{(\partial)}$ generated by $u$ with Galois group $\mathbb{Z} / p \mathbb{Z}$. If $K$ does not contain all $p$-th roots of unity, we may still define $K^{(\partial)}$ by first constructing $\left(K\left(\mu_{p}\right)\right)^{(\partial)}$ and then applying the Galois descent; in this case, the extension $K / K^{(\partial)}$ may not be Galois.

We call the inclusion $\varphi^{(\partial) *}: K^{(\partial)} \hookrightarrow K$ the $\partial$-Frobenius morphism (homomorphism). We view $K^{(\partial)}$ as being equipped with the derivation $\partial^{\prime}=\partial /\left(p u^{p-1}\right)$; it is a derivation on $K^{(\partial)}$ because a simple calculation shows that $(\partial(x))^{(i)}=\zeta_{p}^{i} \partial\left(x^{(i)}\right)$ for any $x \in K$, yielding that $\partial^{\prime}(x)$ is invariant under the $\mathbb{Z} / p \mathbb{Z}$-action if $x \in K^{(\partial)}$. By [Kedlaya and Xiao 2010, Lemma 1.4.9], $\partial^{\prime}$ is of rational type on $K^{(\partial)}$.

We sometimes use $\varphi^{(\partial, n)}: K^{(\partial, n)} \hookrightarrow K$ to denote the $p^{n}$-th $\partial$-Frobenius (homomorphism) obtained by applying the above construction $n$ times; if $K$ contains a primitive $p^{n}$-th root of unity $\zeta_{p^{n}}$, this is the same as the fixed field for the natural action of $\mathbb{Z} / p^{n} \mathbb{Z}$ on $K$ given by $x^{(i)}=\mathbb{T}\left(x ; \partial ;\left(\zeta_{p^{n}}^{i}-1\right) u\right)$ for $i \in \mathbb{Z} / p^{n} \mathbb{Z}$.

Remark 1.2.15. We point out that the definitions of $\partial$-Frobenius and $K^{(\partial)}$ depend on the choice of the rational parameter $u$.

Lemma 1.2.16. The residue field $\kappa_{K^{(\partial)}}$ contains $\kappa_{K}^{p}$.

Proof. We know that $K$ is generated by $u$ over $K^{(\partial)}$. If $|u| \notin\left|K^{(\partial) \times}\right|, K^{(\partial)}$ will have the same residue field as $K$ does. If $|u| \in\left|K^{(\partial) \times}\right|$, let $x \in K^{(\partial)}$ be an element such that $|x|=|u|$. Then $\kappa_{K}$ is generated over $\kappa_{K^{(\partial)}}$ by $\overline{u / x}$, whose $p$-th power lies in $\kappa_{K^{(\partial)}}$. The statement follows.

Definition 1.2.17. Given a $\partial^{\prime}$-differential module $V^{\prime}$ over $K^{(\partial)}$, its $\partial$-Frobenius pullback is the $\partial$-differential module $\varphi^{(\partial) *} V^{\prime}=V^{\prime} \otimes_{K^{(\partial)}} K$ over $K$, where

$$
\partial\left(\boldsymbol{v}^{\prime} \otimes x\right)=p u^{p-1} \partial^{\prime}\left(\boldsymbol{v}^{\prime}\right) \otimes x+\boldsymbol{v}^{\prime} \otimes \partial(x) \quad\left(\boldsymbol{v}^{\prime} \in V^{\prime}, x \in K\right) .
$$

For a $\partial$-differential module $V$ over $K$, we define the $\partial$-Frobenius descendant of $V$ to be the $K^{(\partial)}$-module $\varphi_{*}^{(\partial)} V$ obtained from $V$ by restriction along $\varphi^{(\partial) *}$ : $K^{(\partial)} \rightarrow K$ and viewed as a $\partial^{\prime}$-differential module over $K^{(\partial)}$ with the action given by $\partial^{\prime}(v)=\partial(v) / p u^{p-1}$ for any $v \in V$.

Let $V$ be a $\partial$-differential module over $K$ such that $I R_{\partial}(V)>p^{-1 /(p-1)}$. A $\partial$ Frobenius antecedent of $V$ (which always exists as is shown in Lemma 1.2.18(c)) is a $\partial^{\prime}$-differential module $V^{\prime}$ over $K^{(\partial)}$ such that $V \cong \varphi^{(\partial) *} V^{\prime}$ and $I R_{\partial^{\prime}}\left(V^{\prime}\right)>p^{-p /(p-1)}$. 
Lemma 1.2.18. The d-Frobenius pullbacks and descendants have the following properties.

(a) For $V^{\prime}$ a $\partial^{\prime}$-differential module over $K^{(\partial)}$, we have

$$
I R_{\partial}\left(\varphi^{(\partial) *} V^{\prime}\right) \geq \min \left\{I R_{\partial^{\prime}}\left(V^{\prime}\right)^{1 / p}, p I R_{\partial^{\prime}}\left(V^{\prime}\right)\right\} .
$$

Moreover, if $I R_{\partial^{\prime}}\left(V^{\prime}\right) \neq p^{-p /(p-1)}$, the above inequality is in fact an equality.

(b) For $V$ a d-differential module over $K$, there is a canonical isomorphism $\varphi^{(\partial) *} \varphi_{*}^{(\partial)} V \cong V^{\oplus p}$.

(c) For $i=0, \ldots, p-1$, let $W_{i}^{(\partial)}$ be the $\partial^{\prime}$-differential module over $K^{(\partial)}$ with one generator $\boldsymbol{v}$ (which is a proxy of $\left.u^{i}\right)$, such that $\partial^{\prime}(\boldsymbol{v})=(i / p) u^{-p} \boldsymbol{v}$. Then $I R_{\partial^{\prime}}\left(W_{i}^{(\partial)}\right)=p^{-p /(p-1)}$ for $i=1, \ldots, p-1$. For any $\partial$-differential module $V$ over $K$, we have canonical isomorphisms $\iota_{i}:\left(\varphi_{*}^{(\partial)} V\right) \otimes W_{i}^{(\partial)} \cong \varphi_{*}^{(\partial)} V$ for $i=0, \ldots, p-1$. Moreover, a submodule $U$ of $\varphi_{*}^{(\partial)} V$ is itself the $\partial$ Frobenius descendant of a submodule of $V$ if and only if $\iota_{i}\left(U \otimes W_{i}^{(\partial)}\right)=U$ for $i=0, \ldots, p-1$.

For $V_{1}$ and $V_{2}$ d-differential modules over $K$, we have

$$
\varphi_{*}^{(\partial)} V_{1} \otimes \varphi_{*}^{(\partial)} V_{2}=\left(\varphi_{*}^{(\partial)}\left(V_{1} \otimes V_{2}\right)\right)^{\oplus p} .
$$

For $V^{\prime}$ a $\partial^{\prime}$-differential module over $K^{(\partial)}$, we have

$$
\varphi_{*}^{(\partial)} \varphi^{(\partial) *} V^{\prime} \cong V^{\prime} \oplus \bigoplus_{i=1}^{p-1} V^{\prime} \otimes W_{i}^{(\partial)} .
$$

(d) (Christol-Dwork) Let $V$ be a d-differential module over $K$ such that

$$
I_{\partial}(V)>p^{-1 /(p-1)} .
$$

Then there exists a unique $\partial$-Frobenius antecedent $V^{\prime}$ of $V$. Moreover, we have $I R_{\partial^{\prime}}\left(V^{\prime}\right)=I R_{\partial}(V)^{p}$.

(e) Let $V$ be a d-differential module over $K$. Then

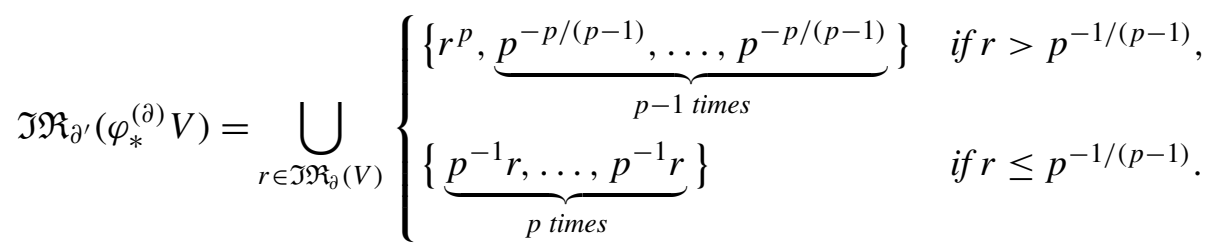

In particular, we have $I R_{\partial^{\prime}}\left(\varphi_{*}^{(\partial)} V\right)=\min \left\{p^{-1} I R_{\partial}(V), p^{-p /(p-1)}\right\}$.

Proof. For (a), see [Kedlaya and Xiao 2010, Lemma 1.4.11 and Corollary 1.4.20].

(b) and (c) are straightforward. For (d), see [Kedlaya 2010, Theorem 10.4.2]. For (e), see [Kedlaya and Xiao 2010, Theorem 1.4.19]. 
Remark 1.2.19. As in [Kedlaya 2010, Theorem 10.4.4], one can form a version of Lemma 1.2.18(d) for differential modules over discs or annuli.

For the following theorem, we do not assume $p>0$.

Theorem 1.2.20. Let $V$ be a d-differential module over $K$. Then there exists a unique decomposition of a-differential modules:

$$
V=\bigoplus_{r \in(0,1]} V_{r}
$$

where every subquotient of $V_{r}$ has pure intrinsic d-radii $r$. Moreover, $V_{r}=0$ if $r \notin\left|K^{\times}\right|^{\mathbb{Q}}$.

Proof. For the decomposition, see [Kedlaya and Xiao 2010, Theorem 1.4.21]. The rationality of those $r$ such that $V_{r} \neq 0$ follows from Proposition 1.2.8 when $r<\omega$ and from taking $\partial$-Frobenius antecedents in the general case.

Definition 1.2.21. We call $\oplus_{r \in(0, \omega)} V_{r}$ the visible part of $V$ and $\oplus_{r \in[\omega, 1]} V_{r}$ the nonvisible part of $V$. If $V$ consists of only its visible part, we say $V$ has visible (intrinsic) $\partial$-radii; similarly, if $V$ consists of only its nonvisible part, we say $V$ has nonvisible (intrinsic) $\partial$-radii.

Remark 1.2.22. Let $V$ be a $\partial$-differential module over $K$ with pure intrinsic $\partial$-radii $I R_{\partial}(V)>p^{-1 /(p-1)}$. By Lemma 1.2.18(d), $V$ has a $\partial$-Frobenius antecedent $V^{\prime}$. By Lemma 1.2.18(c),

$$
\varphi_{*}^{(\partial)} V=\varphi_{*}^{(\partial)} \varphi^{(\partial) *} V^{\prime} \cong V^{\prime} \oplus\left(\bigoplus_{i=1}^{p-1} V^{\prime} \otimes W_{i}^{(\partial)}\right) .
$$

This decomposition coincides with the one obtained by applying Theorem 1.2.20 to $\varphi_{*}^{(\partial)} V$.

1.3. Refined radii. When a $\partial$-differential module $V$ has pure $\partial$-radii, we will define the multiset of refined $\partial$-radii, certain secondary information for the differential module. Similar to the case of $\partial$-radii, we may canonically write $V$ as a direct sum of $\partial$-differential submodules such that the multiset of refined $\partial$-radii for each direct summand consists of elements pairwise-conjugate under the action of $\operatorname{Gal}\left(K^{\mathrm{alg}} / K\right)$.

Hypothesis 1.3.1. In this subsection, let $K$ be a complete nonarchimedean field of characteristic zero and residual characteristic $p$ (possibly $p=0$ ), equipped with a derivation $\partial$ of rational type. We fix $u \in K$ a rational parameter for $\partial$. Unless otherwise specified, we assume that $V$ is a $\partial$-differential module of rank $d$ over $K$ with pure intrinsic $\partial$-radii $I R_{\partial}(V)$. Put $s=-\log \left(\omega R_{\partial}(V)^{-1}\right)=-\log |\partial|_{\mathrm{sp}, V}$. 
Notation 1.3.2. For $P(T)=T^{d}+a_{1} T^{d-1}+\cdots+a_{d} \in K[T]$ a polynomial whose Newton polygon has pure slope $s$, the multiset of the reduced roots of $P$ consists of the reductions of the roots of $P$ in $\kappa_{K^{\text {alg }}}^{(s)}$, counted with multiplicity. If $P$ is the characteristic polynomial of a matrix $A \in \operatorname{Mat}\left(\mathfrak{m}_{K}^{(s)}\right)$, we call the reduced roots of $P$ the reduced eigenvalues of $A$.

Notation 1.3.3. For $\boldsymbol{b} \in(0,1]$ (a proxy of $I R_{\partial}(V)$ ), we define $\lambda=\lambda(\boldsymbol{b})$ and $r=r(\boldsymbol{b})$ as follows.

(i) When $\boldsymbol{b}<\omega$ (which happens if $V$ has pure visible intrinsic $\partial$-radii), we let $\lambda(\boldsymbol{b})=0$ and $r(\boldsymbol{b})=1$.

(ii) When $\boldsymbol{b} \in[\omega, 1)$ and hence $p>0$ (which happens if $V$ has pure nonvisible $\partial$-radii), let $\lambda(\boldsymbol{b})$ denote the unique positive integer such that

$$
\boldsymbol{b} \in\left[p^{\frac{-1}{p^{\lambda(b)-1}(p-1)}}, p^{\frac{-1}{p^{\lambda(b)}(p-1)}}\right)
$$

and put $r(\boldsymbol{b})=p^{\lambda(\boldsymbol{b})}$.

(iii) When $\boldsymbol{b}=1$, we let $\lambda(\boldsymbol{b})=r(\boldsymbol{b})=\infty$.

Definition 1.3.4. Let $\boldsymbol{b} \in(0,1]$. A $K$-norm $|\cdot|_{V}$ on $V$ is called $\boldsymbol{b}$-good (or simply good if $b=I R_{\partial}(V)$ ), if it admits an orthogonal (not necessarily orthonormal) basis, and

(i) when $\boldsymbol{b}<\omega$ (which happens when $\boldsymbol{b}=I R_{\partial}(V)$ for $V$ visible), we have $|\partial|_{V} \leq$ $\omega(\boldsymbol{b}|u|)^{-1}$,

(ii) when $\boldsymbol{b} \in[\omega, 1)$ and hence $p>0$ (which happens when $\boldsymbol{b}=I R_{\partial}(V)$ for $V$ nonvisible), we have

$$
\left|\frac{\partial^{i}}{i !}\right|_{V} \leq|\partial|_{K}^{i} \text { for } i=1, \ldots, r-1, \quad\left|\frac{\partial^{r}}{r !}\right|_{V} \leq p^{-1 /(p-1)}(\boldsymbol{b}|u|)^{-r},
$$

(iii) when $\boldsymbol{b}=1$, we have $\left|\partial^{i} / i !\right|_{V} \leq|\partial|_{K}^{i}$ for all $i \geq 0$.

One may summarize the conditions (i)-(iii) by writing

(iv) $\left|\partial^{i} / i !\right|_{V} \leq \max \left\{|\partial|_{K}^{i},\left(\omega \boldsymbol{b}^{-1}|u|^{-1}\right)^{i} /|i !|\right\}$ for $i=1, \ldots, r$.

Indeed, the equivalence of (1) or (iii) with (iv) is straightforward and the equivalence of (ii) and (iv) (when necessarily $p>0$ ) follows from the observation that the maximum above is equal to $|\partial|_{K}^{i}$ if $i<r$ and to $p^{-1 /(p-1)}(\boldsymbol{b}|u|)^{-r}$ if $i=r$. From condition (iv), it is obvious that a $\boldsymbol{b}$-good norm is also $\boldsymbol{b}^{\prime}$-good for any $\boldsymbol{b}^{\prime} \leq \boldsymbol{b}$.

For the rest of this definition, we assume that $b=I R_{\partial}(V)<1$. By Lemma 1.3.9 below there exists a good norm for $V$.

Using this good norm, we define the multiset of refined $\partial$-radii of $V$, denoted by $\Theta_{\partial}(V)$, as follows. Enlarge the value group of $K$ in the sense of Remark 1.2.11 so that $V$ admits an orthonormal basis. Let $N_{r}$ be the matrix of $\partial^{r}$ with respect to the 
chosen basis. If $\alpha_{1}, \ldots, \alpha_{d}$ are the reduced eigenvalues of $N_{r}$, viewed as elements in $\kappa_{K^{\text {alg }}}^{(r s)}$, we put

$$
\Theta_{\partial}(V,|\cdot|)=\left\{\alpha_{1}^{1 / r}, \ldots, \alpha_{d}^{1 / r}\right\}
$$

as the multiset consisting of elements in $\kappa_{K^{\text {alg }}}^{(s)}$ (note that there is no ambiguity of taking $r$-th roots for elements in $\kappa_{K^{\text {alg }}}^{(r s)}$ when $p>0$ ). We will see in Lemmas 1.3.11 and 1.3.12 that the multiset of refined $\partial$-radii is independent of the choices of the good norm and the orthonormal basis of $V$. After these lemmas, we will abbreviate $\Theta_{\partial}(V,|\cdot|)$ to $\Theta_{\partial}(V)$. When $\Theta_{\partial}(V)$ is pure as a multiset, we say that $V$ has pure refined $\partial$-radii.

We remark that $\Theta_{\partial}(V)$ does not depend on the choice of the rational parameter $u$. But it is sometimes convenient to use the multiset of intrinsic refined $\partial$-radii $\mathscr{I} \Theta_{\partial}(V)=u \Theta_{\partial}(V)$ for a fixed rational parameter $u \in K$.

Finally, in the case when $I_{\partial}(V)=1$, we conventionally define $\Theta_{\partial}(V)$ and $\Phi \Theta_{\partial}(V)$ to be the multisets consisting of 0 with multiplicity $\operatorname{dim} V$.

Remark 1.3.6. In the definition of refined $\partial$-radii, we first enlarged $K$ to $K^{\prime}$, the completion of $K\left(x_{1}, \ldots, x_{n}\right)$ for some $\left(\eta_{1}, \ldots, \eta_{n}\right)$-Gauss norm. However, the multiset of refined $\partial$-radii $\Theta_{\partial}(V,|\cdot|)$ is still composed of elements in $\kappa_{K}^{(s)}$ alg. Indeed, since the construction is canonical, for any $\theta \in \Theta_{\partial}(V,|\cdot|)$, we have $g \theta \in \Theta_{\partial}(V,|\cdot|)$ for any automorphism $g$ of $K^{\prime}$ fixing $K$. But $\Theta_{\partial}(V,|\cdot|)$ is a finite multiset. So it can consist only of elements in $\kappa_{K^{\text {alg }}}^{(s)}$. Alternatively, we can carefully keep track of the new variables we introduced in the computation of reduced eigenvalues; from this, we can also see that the multiset of refined $\partial$-radii is composed of elements in $\kappa_{K^{\text {alg }}}^{(s)}$.

Remark 1.3.7. We also remark that when $p>0$ and $\boldsymbol{b}=\omega^{1 / p^{\lambda}}$, the condition (1.3.5) for $i=1, \ldots, p^{\lambda-1}$ is equivalent to (1.3.5) for $i=1, \ldots, p^{\lambda}$. But we need the matrix $N_{p^{\lambda}}$ to define refined $\partial$-radii. For example, when $\boldsymbol{b}=I R_{\partial}(V)=\omega$, we will see in Lemma 1.3.9 below that the twisted polynomial from Proposition 1.2.8 gives us a good norm on $V$. However, one cannot compute the refined $\partial$-radii by taking the reduced roots of this twisted polynomial. Instead, one has to find the matrix for $\partial^{p}$.

Remark 1.3.8. For a good norm, one can show that the inequalities in (1.3.5) are in fact equalities, but we will not use this fact later; see [Kedlaya 2010, Lemma 6.2.4] for a proof of similar flavor.

Lemma 1.3.9. Let $V$ be as in Hypothesis 1.3.1. Assume that $\boldsymbol{b} \leq I R_{\partial}(V)$, and that $\boldsymbol{b}<1$ if $p>0$. Then $V$ admits a $\boldsymbol{b}$-good norm. In particular, any $V$ with pure intrinsic radii $I R_{\partial}(V)<1$ admits a good norm.

Proof. We first assume that $\boldsymbol{b} \leq \omega$. We take a cyclic vector $\boldsymbol{v} \in V$ with twisted polynomial $P$. By Proposition 1.2.8, the lesser of $-\log |\partial|_{K}$ and the least slope of the 
Newton polygon of $P$ equals $\min \left\{s,-\log |\partial|_{K}\right\} \geq-\log \left(\omega \boldsymbol{b}^{-1}|u|^{-1}\right)$. Then we can define a $\boldsymbol{b}$-good norm on $V$ by taking the orthogonal basis to be $\boldsymbol{v}, \partial \boldsymbol{v}, \ldots, \partial^{d-1} \boldsymbol{v}$ with $\left|\partial^{i} \boldsymbol{v}\right|=\omega^{i}(\boldsymbol{b}|u|)^{-i}$ for $i=0, \ldots, d-1$. When $\boldsymbol{b}=\omega$, as pointed out in Remark 1.3.7, our bound $|\partial|_{V} \leq|u|^{-1}$ alone implies condition (1.3.5) for $r=$ $1, \ldots, p$ when $p>0$, and condition (iii) in Definition 1.3.4 when $p=0$.

The remaining case is when $p>0$ and $\boldsymbol{b} \in\left(p^{-1 /(p-1)}, 1\right)$. We let $n=\lambda-1$ if $\boldsymbol{b}=p^{-1 /\left(p^{\lambda-1}(p-1)\right)}$ and $n=\lambda$ otherwise. In other words, $n$ is the unique nonpositive integer such that

$$
\boldsymbol{b} \in\left(p^{\frac{-1}{p^{n-1}(p-1)}}, p^{\frac{-1}{p^{n}(p-1)}}\right]
$$

Let $\varphi^{(\partial, n)}: K^{(\partial, n)} \rightarrow K$ be the $p^{n}$-th $\partial$-Frobenius and let $\tilde{\partial}=\partial /\left(p^{n} u^{p^{n}-1}\right)$ be the corresponding derivation on $K^{(\partial, n)}$. Since $I R_{\partial}(V) \geq \boldsymbol{b}>p^{-1 /\left(p^{n-1}(p-1)\right)}$, by repeatedly applying Lemma 1.2.18(d), we obtain an $n$-fold $\partial$-Frobenius antecedent $W$ over $K^{(\partial, n)}$; it has intrinsic $\tilde{\partial}$-radii

$$
I R_{\tilde{\partial}}(W)=I R_{\partial}(V)^{p^{n}} \geq \boldsymbol{b}^{p^{n}} \in\left(p^{\frac{-p}{p-1}}, p^{\frac{-1}{p-1}}\right] .
$$

In particular, $W$ has a $\boldsymbol{b}^{p^{n}}$-good norm by the argument in the previous paragraph. We have

$$
\begin{aligned}
& \left|u^{p^{n}} \tilde{\partial}\right|_{W} \leq p^{-1 /(p-1)} \boldsymbol{b}^{-p^{n}} \in[1, p) \\
& \Rightarrow|u \partial|_{W}=p^{-n}\left|u^{p^{n}} \tilde{\partial}\right|_{W}\left\{\begin{array}{l}
<p^{-n} \cdot p=p^{\lambda-1} \\
\leq p^{-n} \cdot 1=p^{\lambda-1}
\end{array} \text { when } n=\lambda,\right.
\end{aligned}
$$

This norm on $W$ gives rise to a $K$-norm $|\cdot|_{V}$ on $V$, which we will show is $\boldsymbol{b}$-good. By (1.3.10), we have $|u \partial-i|_{V}=|u \partial-i|_{W} \leq|i|$ for $i=1, \ldots, p^{\lambda}-1$. Hence we have, for $i=1, \ldots, p^{\lambda}$,

$$
\begin{aligned}
& \left|\frac{u^{i} \partial^{i}}{i !}\right|_{V}=\left|\frac{u^{i} \partial^{i}}{i !}\right|_{W}=\left|\frac{u \partial(u \partial-1) \cdots(u \partial-(i-1))}{i !}\right|_{W} \leq\left|\frac{u \partial}{i}\right|_{W} \\
& \quad=\left|\frac{p^{n}}{i} u^{p^{n}} \tilde{\partial}\right|_{W} \begin{cases}\leq 1 & \text { if } i=1, \ldots, p^{\lambda}-1, \\
\leq p^{-1 /(p-1)} b^{-p^{n}}=p^{-1 /(p-1)} b^{-p^{\lambda}} & \text { if } i=p^{\lambda} \text { and } n=\lambda, \\
\leq p^{-p /(p-1)} b^{-p^{n}}=p^{-1} & \text { if } i=p^{\lambda} \text { and } n=\lambda-1 .\end{cases}
\end{aligned}
$$

This verifies (1.3.5).

Lemma 1.3.11. Assume that $I R_{\partial}(V)<1$. Let $|\cdot|$ be a good norm on $V$. Then the multiset of refined $\partial$-radii $\Theta_{\partial}(V,|\cdot|)$ is well-defined.

Proof. By possibly enlarging $K$ in the sense of Remark 1.2.11, we have two orthonormal bases $\underline{\boldsymbol{e}}$ and $\underline{\boldsymbol{e}}^{\prime}$ for $|\cdot|_{V}$ such that $\underline{\boldsymbol{e}}^{\prime}=\underline{\boldsymbol{e}} A$ for a transition matrix $A \in \mathrm{GL}_{d}\left(\mathrm{O}_{K}\right)$. For $i=1, \ldots, r$, let $N_{i}$ denote the matrix of $\partial^{i}$ with respect to $\underline{\boldsymbol{e}}$; by (1.3.5), we 
have $\left|N_{i} / i !\right| \leq|\partial|_{K}^{i}$ for $i=1, \ldots, r-1$. Then

$$
\frac{\partial^{r}\left(\underline{\left.\boldsymbol{e}^{\prime}\right)}\right.}{r !}=\frac{\partial^{r}(\underline{\boldsymbol{e}} A)}{r !}=\sum_{i=0}^{r} \frac{\partial^{i}(\underline{\boldsymbol{e}})}{i !} \frac{\partial^{r-i}(A)}{(r-i) !}=\underline{\boldsymbol{e}}^{\prime} A^{-1}\left(\sum_{i=0}^{r} \frac{N_{i}}{i !} \frac{\partial^{r-i}(A)}{(r-i) !}\right) .
$$

If $A^{-1} M A$ denote the matrix of $\partial^{r} / r$ ! with respect to $\underline{\boldsymbol{e}}^{\prime}$, we have

$$
M=\frac{N_{r}}{r !}+\sum_{i=0}^{r-1} \frac{N_{i}}{i !} \frac{\partial^{r-i}(A) A^{-1}}{(r-i) !} .
$$

Note that $\left|N_{i} / i !\right| \leq|\partial|_{K}^{i}$ and

$$
\left|\partial^{r-i}(A) A^{-1} /(r-i) !\right| \leq|\partial|_{K}^{r-i}|A|\left|A^{-1}\right| \leq|\partial|_{K}^{r-i}
$$

imply that $\left|M-N_{r} / r !\right| \leq|\partial|_{K}^{r}<\omega R_{\partial}(V)^{-r}$, which is smaller than any singular value of $N_{r} / r$ !. By [Kedlaya 2010, Theorem 4.2.2], the reduced eigenvalues of $N_{r} / r$ ! coincide with those of $A^{-1} M A$. Therefore, $\Theta_{\partial}(V,|\cdot|)$ does not depend on the choice of an orthogonal basis for $|\cdot|$.

Lemma 1.3.12. Assume that $I R_{\partial}(V)<1$. Let $|\cdot|_{1}$ and $|\cdot|_{2}$ be two good norms on $V$. Then $\Theta_{\partial}\left(V,|\cdot|_{1}\right)=\Theta_{\partial}\left(V,|\cdot|_{2}\right)$.

Proof. By possibly enlarging $K$ as in Remark 1.2.11, we may choose orthonormal bases $\underline{\boldsymbol{e}}$ and $\underline{\boldsymbol{f}}$ of $|\cdot|_{1}$ and $|\cdot|_{2}$, respectively, so that $\underline{\boldsymbol{e}} A=\underline{\boldsymbol{f}}$ with $A=$ $\operatorname{Diag}\left\{a_{11}, \ldots, a_{d d}\right\}$.

Let $N_{i}$ denote the matrix of $\partial^{i}$ with respect to $\underline{\boldsymbol{e}}$; by (1.3.5), we have $\left|N_{i} / i !\right| \leq 1$ for $i=1, \ldots, r-1$. Then

$$
\frac{\partial^{r}(\underline{\boldsymbol{f}})}{r !}=\frac{\partial^{r}(\underline{\boldsymbol{e}} A)}{r !}=\sum_{i=0}^{r} \frac{\partial^{i}(\underline{\boldsymbol{e}})}{i !} \frac{\partial^{r-i}(A)}{(r-i) !}=\underline{\boldsymbol{f}} A^{-1}\left(\sum_{i=0}^{r} \frac{N_{i}}{i !} \frac{\partial^{r-i}(A)}{(r-i) !} A^{-1}\right) A .
$$

It suffices to show that $N_{r} / r$ ! has the same reduced eigenvalues as

$$
\sum_{i=0}^{r} \frac{N_{i}}{i !} \frac{\partial^{r-i}(A)}{(r-i) !} A^{-1}
$$

This is true by [Kedlaya 2010, Theorem 4.4.2] since

$$
\begin{aligned}
\left|\frac{N_{i}}{i !} \frac{\partial^{r-i}(A)}{(r-i) !} A^{-1}\right|=\left|\frac{N_{i}}{i !}\right| \cdot \mid \operatorname{Diag}\left(\frac{\partial^{r-i}\left(a_{11}\right)}{(r-i) !} a_{11}^{-1}, \ldots\right. & \left.\frac{\partial^{r-i}\left(a_{d d}\right)}{(r-i) !} a_{d d}^{-1}\right) \mid \\
& \leq|\partial|_{K}^{i} \cdot|\partial|_{K}^{r-i}<\omega R_{\partial}(V)^{-1},
\end{aligned}
$$

for $i=0, \ldots, r-1$.

Corollary 1.3.13. Assume that $V$ has pure visible $\partial$-radii. For any cyclic vector $v \in V$, the multiset of the reduced roots of the twisted polynomial associated to $v$ is 
exactly the multiset of refined $\partial$-radii of $V$. In particular, this multiset is composed of nonzero elements of $\kappa_{K^{\text {alg }}}^{(s)}$.

More generally, we may drop the hypothesis that $V$ has pure $\partial$-radii, and only assume that $V$ has visible $\partial$-radii $R_{\partial}(V)=\omega \mathrm{e}^{s}$. Let $h$ denote the multiplicity of $R_{\partial}(V)$ in the multiset $\mathscr{R}_{\partial}(V)$. In this case, for any cyclic vector $v \in V$, if we write the associated monic twisted polynomial as $X^{d}+a_{1} X^{d-1}+\cdots+a_{d}$, then $\left|a_{i}\right| \leq \mathrm{e}^{-i s}$ for $i \leq h$ and $\left|a_{h}\right|=\mathrm{e}^{-h s}$. Moreover, if $V_{\omega \mathrm{e}^{s}}$ is the direct summand of $V$ with pure $\partial$-radii $\omega \mathrm{e}^{s}$ as given by Theorem 1.2.20, then $\Theta_{\partial}\left(V_{\omega \mathrm{e}^{s}}\right)$ consists of the reduced roots of the polynomial $X^{h}+a_{1} X^{h-1}+\cdots+a_{h}=0$.

Proof. As already pointed out in Remark 1.3.7, we emphasize again that the case $I R_{\partial}(V)=\omega$ is not included in the statement. We first treat the case when $V$ has pure visible $\partial$-radii. We can construct the good norm using the twisted polynomial as in Lemma 1.3.9. This twisted polynomial is then exactly the characteristic polynomial of the matrix of $\partial$ with respect to this basis. The claim follows.

For $V$ not necessarily pure of $\partial$-radii, the bound for norms on $a_{i}$ follows from Proposition 1.2.8. For the statement about refined $\partial$-radii, we need to dig into the proof of Theorem 1.2.20 a bit more. By [Kedlaya 2009, Corollary 3.2.4], we can write $P=Q R$ where $Q$ and $R$ are monic twisted polynomials such that the Newton polygon of $Q=X^{h}+a_{1}^{\prime} X^{h-1}+\cdots+a_{h}^{\prime}$ has pure slopes $s$ and the Newton polygon of $R$ has slope strictly bigger than $s$. Moreover, we have $V_{\omega \mathrm{e}^{s}}=K\{T\} / Q K\{T\}$. The upshot is that the formal multiplication satisfies $\left|a_{i}-a_{i}^{\prime}\right|<\mathrm{e}^{i s}$ for any $i=1, \ldots, h$. Therefore, the reduced roots of $X^{h}+a_{1} X^{h-1}+\cdots+a_{h}=0$ are the same as the reduced roots of $X^{h}+a_{1}^{\prime} X^{h-1}+\cdots+a_{h}^{\prime}=0$, which are the same as the elements of $\Theta_{\partial}(V)$ by the discussion in the previous paragraph.

Lemma 1.3.14. Fix $\boldsymbol{b} \in(0,1)$ and set $r=r(\boldsymbol{b}), \lambda=\lambda(\boldsymbol{b})$, and $s=-\log \left(\omega(\boldsymbol{b}|u|)^{-1}\right)$. Let $V^{\prime}$ be a $\partial$-differential module over $K$ of rank $d$, equipped with a basis $\underline{\boldsymbol{e}}$, with respect to which the action of $\partial$ satisfies the conditions in Definition 1.3.4 with the chosen $\boldsymbol{b}$. Assume that the reduced eigenvalues of the matrix $N_{r} \in \operatorname{Mat}\left(\mathfrak{m}_{K}^{(r s)}\right)$ of $\partial^{r}$ on $V^{\prime}$ are all nonzero in $\kappa_{K^{\text {alg }}}^{(r s)}$. Then $V^{\prime}$ has pure intrinsic $\partial$-radii $\boldsymbol{b}$. As a consequence, $\Theta_{\partial}\left(V^{\prime}\right)$ is exactly the multiset of the reduced eigenvalues of $N_{r}$.

Proof. Since $N_{r} \in \operatorname{Mat}\left(\mathfrak{m}_{K}^{(r s)}\right)$, we have $I R_{\partial}\left(V^{\prime}\right) \geq \boldsymbol{b}$. Suppose that $V^{\prime}$ does not have pure intrinsic $\partial$-radii $\boldsymbol{b}$. Enlarging $K$ as in Remark 1.2.11 if needed, we may apply Theorem 1.2.20 and Lemma 1.3.9 to $V^{\prime}$ and its Jordan-Hölder constituents to find a basis $\boldsymbol{f}$ for which the conditions in Definition 1.3.4 hold and the matrix $\widetilde{N}_{r} \in \operatorname{Mat}\left(\mathfrak{m}_{K}^{(r s)}\right)$ of $\partial^{r}$ is degenerate modulo $\mathfrak{m}_{K^{\text {alg }}}^{(r s)+}$ (when identifying $\kappa_{K}^{(r s)}$ with $\left.\kappa_{K}\right)$. Now, the same argument in the proof of Lemma 1.3.12 implies that $N_{r}$ and $\widetilde{N}_{r}$ must have the same multiset of reduced eigenvalues. But zero is a reduced eigenvalue of $\widetilde{N}_{r}$ but not of $N_{r}$, which is a contradiction. Hence $V^{\prime}$ has pure intrinsic $\partial$-radii $\boldsymbol{b}$. The last statement is Definition 1.3.4. 
Lemma 1.3.15. We have $\Theta_{\partial}\left(V^{\vee}\right)=-\Theta_{\partial}(V)=\left\{-\theta \mid \theta \in \Theta_{\partial}(V)\right\}$.

Proof. This is straightforward.

We will prove in Theorem 1.3.26 a direct sum decomposition of $V$ parametrized by the multiset of refined $\partial$-radii. For this, we start with some basic properties of refined $\partial$-radii when $V$ has visible $\partial$-radii.

Lemma 1.3.16. Let $V$ and $W$ be two d-differential modules over $K$ with pure and visible $\partial$-radii $R_{\partial}(V)=R_{\partial}(W)$. Then the following two statements are equivalent.

(1) The refined $\partial$-radii of $V$ and $W$ are distinct, that is, $\Theta_{\partial}(V) \cap \Theta_{\partial}(W)=\varnothing$.

(2) The tensor product $V \otimes W^{\vee}$ has pure $\partial$-radii $R_{\partial}(V)$.

Moreover, if either statement holds, we have an equality of multisets:

$$
\Theta_{\partial}\left(V \otimes W^{\vee}\right)=\left\{\theta_{1}-\theta_{2}: \theta_{1} \in \Theta_{\partial}(V), \theta_{2} \in \Theta_{\partial}(W)\right\} .
$$

As corollaries, we have the following:

(a) If $\Theta_{\partial}(V) \cap \Theta_{\partial}(W)=\varnothing$, then any homomorphism $f: W \rightarrow V$ of $\partial$-differential modules is zero.

(b) If $\Theta_{\partial}(W)$ has pure refined $\partial$-radii $\theta \in \kappa_{K^{a l g}}^{(s)}$, then $\theta \in \Theta_{\partial}(V)$ if and only if $V \otimes W^{\vee}$ does not have pure $\partial$-radii $R_{\partial}(V)$.

(c) If $\Theta_{\partial}(V)$ and $\Theta_{\partial}(W)$ both have the same pure $\partial$-radii $\theta \in \kappa_{K^{\text {alg }}}^{(s)}$, then we have $R_{\partial}\left(V \otimes W^{\vee}\right)>R_{\partial}(V)$.

Proof. By Lemma 1.3.15, we have $\Theta_{\partial}\left(W^{\vee}\right)=-\Theta_{\partial}(W)$. We may enlarge $K$ as in Remark 1.2.11 so that we have good norms on both $V$ and $W^{\vee}$ given by orthonormal bases. Equip $V \otimes W^{\vee}$ with the tensor product norm. Let $N_{0}, N_{1} \in \operatorname{Mat}\left(\mathfrak{m}_{K}^{(s)}\right)$ be the matrices of $\partial$ acting on $V$ and $W^{\vee}$ with respect to the given bases, respectively. By Definition 1.3.4, $\Theta_{\partial}(V)$ and $-\Theta_{\partial}(W)$ are the multisets of reduced eigenvalues of $N_{0}$ and $N_{1}$, respectively. Then the multiset of reduced eigenvalues of the matrix $N=N_{0} \otimes 1+1 \otimes N_{1}$ is exactly $\left\{\theta_{1}-\theta_{2}: \theta_{1} \in \Theta_{\partial}(V), \theta_{2} \in \Theta_{\partial}(W)\right\}$.

If (1) holds, then all reduced eigenvalues of $N$ are nonzero and hence $\left|N^{n}\right|=\mathrm{e}^{-n s}$ for all $n \in \mathbb{N}$. Moreover, the reduction of $N^{n}$ in $M_{d}\left(\kappa_{K^{\text {alg }}}^{(n s)}\right)$ has full rank if we identify $\kappa_{K^{\text {alg }}}^{(n s)}$ with $\kappa_{K^{\text {alg }}}$. Therefore, $V \otimes W^{\vee}$ has pure $\partial$-radii $R_{\partial}(V)$ by Lemma 1.3.14, proving (2).

Conversely, if (2) holds, then the tensor product norm is a good norm on $V \otimes W^{\vee}$ already and the multiset of reduced eigenvalues of $N$ is the multiset of refined $\partial$-radii of $V \otimes W^{\vee}$. By Corollary 1.3.13, $0 \notin \Theta_{\partial}\left(V \otimes W^{\vee}\right)$. This implies (1).

We now prove (a). Since $V \otimes W^{\vee}$ has pure $\partial$-radii $R_{\partial}(V)<\omega$, we have $H_{\partial}^{0}\left(V \otimes W^{\vee}\right)=0$ and hence there is no nonzero homomorphism of $\partial$-differential modules from $W$ to $V$.

Statement (b) is just (a special case of) the inverse statement of (1) $\Leftrightarrow(2)$. 
For (c), we know that $N_{0}$ and $N_{1}$ have pure reduced eigenvalues $\theta$ and $-\theta$, respectively. Hence $N=N_{0} \otimes 1+1 \otimes N_{1}$ reduces to a matrix in $\kappa_{K^{\text {alg }}}^{(s)}$ with zero eigenvalues (if we identify $\kappa_{K^{\text {alg }}}^{(s)}$ with $\kappa_{K^{\text {alg }}}$ ). It is then nilpotent, that is,

$$
N^{n} \in \operatorname{Mat}\left(\mathfrak{m}_{K^{\text {alg }}}^{(n s)+}\right) \quad \text { for } n \geq \operatorname{dim} V \cdot \operatorname{dim} W .
$$

This implies that $R_{\partial}\left(V \otimes W^{\vee}\right)>R_{\partial}(V)$.

Lemma 1.3.17. Let $V$ and $W$ be two d-differential modules over $K$. Assume that $V$ has pure and visible $\partial$-radii and $R_{\partial}(V)<R_{\partial}(W)$. Then $V \otimes W^{\vee}$ has pure $\partial$-radii $R_{\partial}(V)$ and multiset of refined $\partial$-radii is composed of $\operatorname{dim} W$ copies of $\Theta_{\partial}\left(V \otimes W^{\vee}\right)$.

Proof. By Theorem 1.2.20, we may assume that $W$ has pure $\partial$-radii. By Lemma 1.3.9 we may find a $\boldsymbol{b}$-good norm on $W$ with $\boldsymbol{b}=\min \left\{I R_{\partial}(W), \omega\right\}>I R_{\partial}(V)$.

We proceed as in Lemma 1.3.16. If $N_{0}$ and $N_{1}$ are the matrices of $\partial$ with respect to some orthonormal bases of $V$ and $W^{\vee}$, respectively, then we have $N_{1} \in \operatorname{Mat}\left(\mathfrak{m}_{K}^{(s)+}\right)$ and that $N_{0}$ has pure reduced eigenvalue $\Theta_{\partial}(V)$. Hence the multiset of reduced eigenvalues of $N_{0} \otimes 1+1 \otimes N_{1}$ is simply composed of $\operatorname{dim} W$ copies of the set of reduced eigenvalues of $N_{0}$. The lemma follows.

The refined $\partial$-radii of a nonvisible $\partial$-differential module is closely related to the $\partial^{\prime}$-radii of its Frobenius antecedent; we can save much computation by using this fact. To establish this relation explicitly, it is more convenient to work with the refined intrinsic $\partial$-radii.

Proposition 1.3.18. Assume $p>0$. Let $\varphi^{(\partial)}: K^{(\partial)} \rightarrow K$ be the $\partial$-Frobenius with respect to the parameter $u$.

(a) Assume that $I R_{\partial}(V) \in\left(p^{-1 /(p-1)}, 1\right)$, and then Lemma 1.2.18(d) implies that $V=\varphi^{(\partial) *} W$ for some $\partial^{\prime}$-differential module $W$ on $K^{(\partial)}$ such that

$$
I R_{\partial^{\prime}}(W)=I R_{\partial}(V)^{p} .
$$

We have

$$
\Theta_{\partial}(V)=\left\{-\left(p \theta^{\prime}\right)^{1 / p}: \theta^{\prime} \in \Theta_{\partial^{\prime}}(W)\right\} .
$$

(b) Assume that $I R_{\partial}(V)=p^{-1 /(p-1)}$, and then $\varphi_{*}^{(\partial)}(V)$ has pure intrinsic $\partial^{\prime}$-radii $p^{-p /(p-1)}$. The elements in $\mathscr{I} \Theta_{\partial^{\prime}}\left(\varphi_{*}^{(\partial)}(V)\right)$ can be grouped into p-tuples

$$
\left(\frac{\theta}{p}, \frac{\theta+1}{p}, \ldots, \frac{\theta+p-1}{p}\right)
$$

with $\theta \in \kappa_{K^{\text {alg }}}$, and $\Phi \Theta_{\partial}(V)$ is the multiset composed of $\left(\theta^{p}-\theta\right)^{1 / p} \in \kappa_{K^{\text {alg }}}$ for each p-tuple above. 
(c) Assume $I_{\partial}(V)<p^{-1 /(p-1)}$. Then we have

$$
\mathscr{I} \Theta_{\partial^{\prime}}\left(\varphi_{*}^{(\partial)} V\right)=\{\underbrace{p^{-1} \theta, \ldots, p^{-1} \theta}_{p \text { times }}: \theta \in \mathscr{I} \Theta_{\partial}(V)\} .
$$

Proof. (a) By Lemma 1.3.9 and by possibly enlarging $K$ as in Remark 1.2.11, we can take an orthonormal basis $\underline{\boldsymbol{e}}$ on $W$ which defines a good norm. The norm induces a good norm on $V$ by the explicit construction in Lemma 1.3.9. Let $\lambda$ be as in Notation 1.3.3. We have

$$
\begin{aligned}
u^{p^{\lambda}} \partial^{p^{\lambda}} & =u \partial(u \partial-1) \cdots\left(u \partial-p^{\lambda}+1\right)=p u^{p} \partial^{\prime}\left(p u^{p} \partial^{\prime}-1\right) \cdots\left(p u^{p} \partial^{\prime}-p^{\lambda}+1\right) \\
& =p^{p^{\lambda-1}} u^{p^{\lambda}} \partial^{\prime p^{\lambda-1}} \prod_{i=1, p \nmid i}^{p^{\lambda}-1}\left(p u^{p} \partial^{\prime}-i\right) ;
\end{aligned}
$$

this operator also acts on $W$. Since $\left|u^{p} \partial^{\prime}\right|_{W} \leq \max \left\{1, p^{-1 /(p-1)} I R_{\partial^{\prime}}(W)\right\}<p$, we have

$$
\left|u^{p^{\lambda}} \partial^{p^{\lambda}}-p^{p^{\lambda-1}}((-1) \cdots(-p+1))^{p^{\lambda-1}} u^{p^{\lambda}} \partial^{\prime p^{\lambda}}\right|_{W}<\left|u^{p^{\lambda}} \partial^{p^{\lambda}}\right|_{W}
$$

Therefore, the matrix of $\partial^{p^{\lambda}}$ with respect to $\underline{\boldsymbol{e}}$ is congruent to the matrix of

$$
(-1)^{p^{\lambda-1}(p-1)}(p !)^{p^{\lambda-1}} \partial^{\prime p^{\lambda-1}} \quad \text { modulo } \mathfrak{m}_{K}^{\left(p^{\lambda} s\right)+} \text {. }
$$

We then must have

$\Theta_{\partial}(V,|\cdot|)=\left\{\left((-1)^{(p-1)}(p !) \theta\right)^{1 / p} \mid \theta \in \Theta_{\partial^{\prime}}(W)\right\}=\left\{-(p \theta)^{1 / p} \mid \theta \in \Theta_{\partial^{\prime}}(W)\right\}$.

(b) When $I R_{\partial}(V)=p^{-1 /(p-1)}$, Lemma 1.2.18(e) implies that $\varphi_{*}^{(\partial)} V$ has pure intrinsic $\partial^{\prime}$-radii $p^{-p /(p-1)}$. By Lemma 1.2.18(e) and Lemma 1.3.16, the elements in $\mathscr{I} \Theta_{\partial^{\prime}}\left(\varphi_{*}^{(\partial)}(V)\right)$ can be grouped into $p$-tuples

$$
\left(\frac{\theta}{p}, \frac{\theta+1}{p}, \ldots, \frac{\theta+p-1}{p}\right)
$$

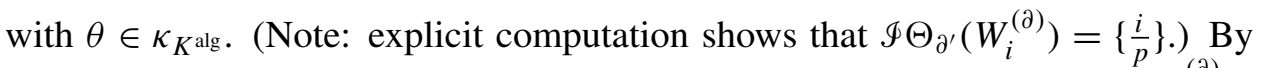
possibly enlarging $K$ in the sense of Remark 1.2.11, we may assume that $\varphi_{*}^{(\partial)} V$ admits a good norm defined by an orthonormal basis $\underline{\boldsymbol{e}}$. Let $N$ be the matrix of $p u^{p} \partial^{\prime}$ with respect to $\underline{\boldsymbol{e}}$. Then $u^{p} \partial^{p}$ acts on $\varphi^{(\partial) *} \varphi_{*}^{(\partial)}(V)=V^{\oplus p}$ according to

$$
u \partial(u \partial-1) \cdots(u \partial-p+1)=p u^{p} \partial^{\prime}\left(p u^{p} \partial^{\prime}-1\right) \cdots\left(p u^{p} \partial^{\prime}-p+1\right) .
$$

Hence the matrix for this action with respect to $\underline{\boldsymbol{e}}$ is congruent to the product $N(N-1) \cdots(N-p+1)$ modulo $p \widehat{O}_{K^{(\partial)}}$ since $\left|p u^{p} \partial^{\prime}\right|_{K^{(\partial)}}=p^{-1}$; then the multiset of its reduced eigenvalues is composed of $\theta^{p}-\theta$ with multiplicity $p$ for each tuple

$$
\left(\frac{\theta}{p}, \frac{\theta+1}{p}, \ldots, \frac{\theta+p-1}{p}\right)
$$


in the multiset of reduced eigenvalues of $N$. The statement follows.

(c) By Lemma 1.2.18(e), $\varphi_{*}^{(\partial)} V$ has pure intrinsic $\partial^{\prime}$-radii

$$
p^{-1} I R_{\partial}(V) \leq p^{-p /(p-1)} .
$$

Since $u^{p} \partial^{\prime}=u \partial / p$, we can take a good norm of $\varphi_{*}^{(\partial)} V$ and deduce that

$$
\Phi \Theta_{\partial^{\prime}}\left(\varphi_{*}^{(\partial)} V\right)=\frac{1}{p} g \Theta_{\partial}\left(\varphi^{(\partial) *} \varphi_{*}^{(\partial)} V\right),
$$

which in turn equals $\frac{1}{p} \oiint_{\partial}\left(V^{\oplus p}\right)$ by Lemma 1.2.18(b). The statement follows.

Proposition 1.3.19. Lemma 1.3.16 holds only assuming $I R_{\partial}(V)=I R_{\partial}(W)<1$ instead of the visible hypothesis. Similarly, Lemma 1.3.17 holds with only assuming $I R_{\partial}(V)<1$ instead of the visible hypothesis on $V$.

Proof. It suffices to check the remaining cases: $p>0$ and $I R_{\partial}(V) \geq p^{-1 /(p-1)}$. If $I R_{\partial}(V)>p^{-1 /(p-1)}$, the statements for $V$ and $W$ follow from the statements on their $\partial$-Frobenius antecedents by Proposition 1.3.18(a). If $I R_{\partial}(V)=p^{-1 /(p-1)}$, the statements for $V$ and $W$ follow from the statements on their $\partial$-Frobenius descendants by Proposition 1.3.18(b) and Lemma 1.2.18(c).

The following is an example of $\partial$-differential modules with pure refined $\partial$-radii. It will serve as a testing object later.

Our convention is to use Gothic letter $\mathfrak{s}$ instead of $s$ when discussing intrinsic radii of convergence; we will never use both $s$ and $\mathfrak{s}$ together.

Example 1.3.20. Fix $\mathfrak{s} \in-\log \left|K^{\times}\right|^{\mathbb{Q}}$ such that $\mathfrak{s}<0$ if $p=0$, and $\mathfrak{s}<\frac{1}{p} \log p$ if $p>0$. Let $\theta \in \kappa_{K^{\text {alg }}}^{(\mathfrak{s})}$ be a nonzero element.

(1) If $p=0$, then we have $\mathfrak{s} \in-\log \left|\left(K^{\prime}\right)^{\times}\right|$and $\theta \in \kappa_{K^{\prime}}^{(\mathfrak{s})}$ for some finite tamely ramified extension $K^{\prime}$ of $K$. Let $x$ be a lift of $\theta$ to $\mathfrak{m}_{K^{\prime}}^{(\mathfrak{s})}$. Put $d=1$ and $n=0$.

(2) If $p>0$, there exists $n \in \mathbb{N}$ such that $\theta^{p^{n}} \in\left(\kappa_{K^{\prime}}^{\left(p^{n-1} \mathfrak{s}\right)}\right)^{p}$ with $p^{n-1} \mathfrak{s} \in-\log \left|\left(K^{\prime}\right)^{\times}\right|$ for some finite tamely ramified extension $K^{\prime}$ of $K$. By Lemma 1.2.16, we may find a lift

$$
x \in u^{-p^{n}} \mathfrak{m}_{K^{\prime(\partial)}}^{\left(p^{n} \mathfrak{s}\right)}
$$

of $u^{-p^{n}} \theta^{p^{n}}$, where the extra $u^{-p^{n}}$ reflects the different normalizations of refined intrinsic $\partial$-radii and refined $\partial$-radii. Put $d=p^{n}$.

Let $\mathscr{L}_{x,(n)}$ be the $\partial$-differential module over $K^{\prime}$ of rank $d$ with basis $\left\{\boldsymbol{e}_{1}, \ldots, \boldsymbol{e}_{d}\right\}$, where the $\partial$-action is given by $\partial \boldsymbol{e}_{i}=\boldsymbol{e}_{i+1}$ for $i=1, \ldots, d-1$ and $\partial \boldsymbol{e}_{d}=x \boldsymbol{e}_{1}$.

Remark 1.3.21. When $p>0$, we point out that $\mathfrak{s}<\frac{1}{p} \log p$ also includes some part of the nonvisible range. The restriction $\mathfrak{s}<\frac{1}{p} \log p$ in Example 1.3.20 is linked with the choice $x \in u^{-p^{n}} \mathfrak{m}_{K^{\prime(\partial)}}^{\left(p^{n} \mathfrak{s}\right)}$. In general, we may extend the range of $\mathfrak{s}$ to be 


$$
\left(-\infty,\left(\frac{1}{p-1}-\frac{1}{p^{c}(p-1)}\right) \log p\right)
$$

for some $c \in \mathbb{N}$, but the price we pay is to take $x \in u^{-p^{n}} \mathfrak{m}_{K^{\prime(\partial, c)}}^{\left(p^{n} \mathfrak{s}\right)}$ lifting $u^{-p^{n}} \theta^{p^{n}}$ for some $n \in \mathbb{N}$ and some finite tamely ramified extension $K^{\prime}$ of $K$. However, as $c$ gets larger, we need to enlarge $n$ to guarantee the existence of such a lift $x$. This is why we may not assume that $\mathfrak{s}<\frac{1}{p-1} \log p$.

Remark 1.3.22. In the nonvisible case, one can construct a $\partial$-differential module with pure refined $\partial$-radii by simply pulling back a $\partial^{\prime}$-differential module over $K^{(\partial)}$ with appropriate refined $\partial^{\prime}$-radii. However, such a naïve construction does not help our later study of the one-dimensional variation of refined $\partial$-radii. We will construct Example 1.5.7, a family version of Example 1.3.20, which looks similar in both visible and nonvisible ranges.

Lemma 1.3.23. Keep the notation as in Example 1.3.20. Then $\mathscr{L}_{x,(n)}$ has pure intrinsic $\partial$-radii $I R_{\partial}\left(\mathscr{L}_{x,(n)}\right)=\omega \mathrm{e}^{\mathfrak{s}}$ and pure refined intrinsic $\partial$-radii $\theta$.

Proof. We may replace $K$ by the completion of $K(z)$ with respect to the $|u|^{-1} \mathrm{e}^{-\mathfrak{s}_{-}}$ Gauss norm (and set $\partial z=0$ ).

We first assume that either we have $p=0$ or we have $p>0$ and $\mathfrak{s}<0$, that is, we consider the visible $\partial$-radii case. We note that $\boldsymbol{e}_{1}, z^{-1} \boldsymbol{e}_{2}, \ldots, z^{-(d-1)} \boldsymbol{e}_{d}$ together define a good norm on $\mathscr{L}_{x,(n)}$; it is a straightforward computation to check that the statement in this case.

We now tackle the case when $p>0$ and $\mathfrak{s} \in\left[0, \frac{1}{p} \log p\right)$. For $i=1, \ldots, p$, we have

$$
\partial^{i} \boldsymbol{e}_{l}=\boldsymbol{e}_{i+l} \text { when } i+l \leq p^{n}, \quad \text { and } \partial^{i} \boldsymbol{e}_{p^{n}-l}=\partial^{i-l}\left(x \boldsymbol{e}_{1}\right) \text { when } i \geq l .
$$

We will show that $\left\{\boldsymbol{e}_{1}, z^{-1} \boldsymbol{e}_{2}, \ldots, z^{-\left(p^{n}-1\right)} \boldsymbol{e}_{p^{n}}\right\}$ defines a good norm on $\mathscr{L}_{x,(n)}$. Indeed, for $i=1, \ldots, p$, the matrix of $\partial^{i}$ with respect to this basis is

$$
N_{i}=\left(\begin{array}{ccccccc}
0 & 0 & \cdots & z^{i} & 0 & \cdots & 0 \\
0 & 0 & \cdots & 0 & z^{i} & \cdots & 0 \\
\vdots & \vdots & \ddots & \vdots & \vdots & \ddots & \vdots \\
0 & 0 & \cdots & 0 & 0 & \cdots & z^{i} \\
z^{-p^{n}+i} x & 0 & \cdots & 0 & 0 & \cdots & 0 \\
z^{-p^{n}+i} \partial x & z^{-p^{n}+i} x & \cdots & 0 & 0 & \cdots & 0 \\
\vdots & \vdots & \ddots & \vdots & \vdots & \ddots & \vdots \\
z^{-p^{n}+i} \partial^{i-1} x & (i-1) z^{-p^{n}+i} \partial^{i-2} x & \cdots & 0 & 0 & \cdots & 0
\end{array}\right)
$$

Note that

$$
|\partial|_{K^{(\partial)}}=p^{-1}|u|^{p-1}\left|\partial^{\prime}\right|_{K^{(\partial)}}=p^{-1}|u|^{-1} \leq \omega|z|<|z| .
$$


Hence, modulo $\mathfrak{m}_{K}^{(-\log |z|)+}$, the nonzero terms of $N_{i}$ are the $z^{i}$ and $z^{-p^{n}+i} x$ in (1.3.24); they form a 2-by-2 block matrix

$$
\bar{N}_{i}^{(-\log |z|)}=\left(\begin{array}{cc}
0 & z^{i} \cdot I_{\left(p^{n}-i\right) \times\left(p^{n}-i\right)} \\
z^{-p^{n}+i} x \cdot I_{i \times i} & 0
\end{array}\right) \in \operatorname{Mat}_{p^{n} \times p^{n}}\left(\kappa_{K}^{(-\log |z|)}\right) .
$$

Note that $\left|z^{-p^{n}+i} x\right|=|z|^{i}$. By Lemma 1.3.14, we have $I R_{\partial}\left(\mathscr{L}_{x,(n)}\right)=\omega \mathrm{e}^{\mathfrak{s}}$ and that this basis defines a good norm on $V$. Moreover, the multiset of reduced eigenvalues of $N_{p}$ is composed of the element $x^{1 / p^{n-1}}$ with multiplicity $p^{n}$. This implies that $\mathscr{I} \Theta_{\partial}(V)=\left\{\theta\right.$ ( $p^{n}$ times $\left.)\right\}$ by the choice of $x$ in Example 1.3.20.

Lemma 1.3.25. Let $V$ be a $\partial$-differential module over $K$ with pure visible $\partial$-radii $R_{\partial}(V)=\omega \mathrm{e}^{s}$. Then we have the following.

(a) For any subquotient $V_{0}$ of $V$, all elements in $\Theta_{\partial}\left(V_{0}\right)$ appear in $\Theta_{\partial}(V)$.

(b) For any $\theta \in \kappa_{K^{\mathrm{alg}}}^{(s)}$, there is a unique maximal d-differential submodule of $V$ which has pure refined $\partial$-radii $\theta$.

Proof. For $\theta \in \kappa_{K^{\text {alg }}}^{(s)}$ such that $\theta \notin \Theta_{\partial}(V)$, let $\mathscr{L}_{x,(n)}$ be the $\partial$-differential module constructed in Example 1.3.20. By Lemmas 1.3.23 and 1.3.16, $V \otimes \mathscr{L}_{x,(n)}^{\vee}$ has pure $\partial$-radii $R_{\partial}(V)$, and so does $V_{0} \otimes \mathscr{L}_{x,(n)}^{\vee}$. By the same lemmas again, we have $\theta \notin \Theta_{\partial}\left(V_{0}\right)$. This proves (a). We point out that this, however, does not prove the inclusion $\Theta_{\partial}\left(V_{0}\right) \subseteq \Theta_{\partial}(V)$ as a multiset, which will be a corollary of Theorem 1.3.26 below.

The second statement follows from the observation that if two submodules $V_{1}$ and $V_{2}$ of $V$ both have pure refined $\partial$-radii $\theta$, so does their sum $V_{1}+V_{2}$ because it is a quotient of $V_{1} \oplus V_{2}$.

Similarly to the direct sum decomposition by intrinsic $\partial$-radii, we have a direct sum decomposition by refined intrinsic $\partial$-radii. The latter is in fact deduced from the former by twisting $\partial$-differential modules of the form $\mathscr{L}_{x,(n)}$.

Theorem 1.3.26. Let $K$ and $V$ be as in Hypothesis 1.3.1. Then $V$ admits a unique direct sum decomposition

$$
V=\bigoplus_{\{\theta\} \subset \kappa_{K^{(s)}}^{\text {alg }}} V_{\{\theta\}},
$$

where the direct sum runs through all $\mathrm{Gal}\left(K^{\mathrm{alg}} / K\right)$-orbits $\{\theta\}$ in $\kappa_{K^{\text {alg }}}^{(s)}$, such that the refined $\partial$-radii of $V_{\{\theta\}}$ is a multiset consisting of the $\operatorname{Gal}\left(K^{\text {alg }} / K\right)$-orbit $\{\theta\}$ with appropriate multiplicities.

Moreover, if $K^{\prime}$ is a finite tamely ramified extension of $K$ such that all the $\theta$ in the above decomposition belong to $\bigcup_{n}\left(\kappa_{K^{\prime}}^{\left(p^{n} s\right)}\right)^{1 / p^{n}}$, then we have a unique direct 
sum decomposition

$$
V \otimes_{K} K^{\prime}=\bigoplus_{\theta \in \kappa_{K^{\prime a l g}}^{(s)}} V_{\theta}
$$

of $\partial$-differential modules over $K^{\prime}$ such that each $V_{\theta}$ has pure refined $\partial$-radii $\theta$.

Proof. The statement is void if $I R_{\partial}(V)=1$. We assume $I R_{\partial}(V)<1$ from now on. We first replace $K$ by the $K^{\prime}$ in the theorem; using the uniqueness of such a direct sum decomposition and Galois descent, we may recover the statement over $K$. Note that Lemma 1.2.10 implies that $\partial$ is still a derivation of rational type.

We first assume that either $p=0$, or $p>0$ and $I R_{\partial}(V)<p^{-1 /(p-1)}$. For each $\theta \in \Theta_{\partial}(V)$, we construct $\mathscr{L}_{x,(n)}$ as in Example 1.3.20, which is a rank $d \partial$-differential module with pure $\partial$-radii $R_{\partial}(V)$ and pure refined radii $\theta$. By Lemma 1.3.16(b), $V \otimes \mathscr{L}_{x,(n)}^{\vee}$ does not have pure radii $R_{\partial}(V)$. Theorem 1.2.20 then gives rise to a decomposition $V \otimes \mathscr{L}_{x,(n)}^{\vee}=W_{0} \oplus W_{1}$, where $R_{\partial}\left(W_{0}\right)>R_{\partial}(V)$ and $W_{1}$ has pure d-radii $R_{\partial}(V)$.

Put $\widetilde{W}_{0}=W_{0} \otimes \mathscr{L}_{x,(n)}$ and $\widetilde{W}_{1}=W_{1} \otimes \mathscr{L}_{x,(n)}$. Consider the following homomorphisms of $\partial$-differential modules:

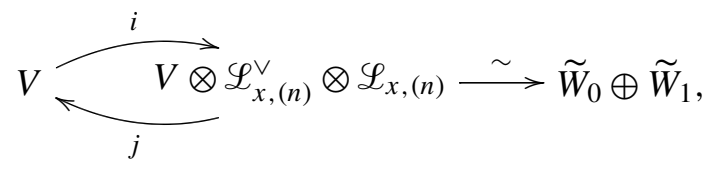

where $i$ is induced by the diagonal embedding $K \hookrightarrow \mathscr{L}_{x,(n)}^{\vee} \otimes \mathscr{L}_{x,(n)}$ and $j$ is induced by the trace map $\mathscr{L}_{x,(n)}^{\vee} \otimes \mathscr{L}_{x,(n)} \rightarrow K$ normalized so that $j i=\mathrm{id}$. Let $p_{0}$ and $p_{1}$ be the projections from $V \otimes \mathscr{L}_{x,(n)}^{\vee} \otimes \mathscr{L}_{x,(n)}$ to the factors $\widetilde{W}_{0}$ and $\widetilde{W}_{1}$, respectively, viewed as submodules of the source. We then have $p_{0}^{2}=p_{0}, p_{1}^{2}=p_{1}$, and $p_{0}+p_{1}=1$.

We claim that $j p_{0} i$ and $j p_{1} i$ are projectors on $V$. Indeed, Lemma 1.3.16(c) implies that $R_{\partial}\left(\mathscr{L}_{x,(n)}^{\vee} \otimes \mathscr{L}_{x,(n)}\right)>R_{\partial}(V)$. By Lemma 1.3.17,V® $\mathscr{L}_{x,(n)}^{\vee} \otimes \mathscr{L}_{x,(n)}$ and hence $\widetilde{W}_{0}$ and $\widetilde{W}_{1}$ have pure $\partial$-radii $R_{\partial}(V)$. Lemma 1.3 .17 also implies that $\Theta_{\partial}\left(\widetilde{W}_{0}\right)$ consists of solely $\theta$, and by the "moreover" part of Lemma 1.3.16, we have

$$
\left.\Theta_{\partial}\left(\widetilde{W}_{1}\right)=\left\{\theta_{1}+\theta \text { (with multiplicity } d\right) \mid \theta_{1} \in \Theta_{\partial}\left(W_{1}\right)\right\} .
$$

In particular, we have $\theta \notin \Theta_{\partial}\left(\widetilde{W}_{1}\right)$. Hence any homomorphism of $\partial$-differential modules between $\widetilde{W}_{0}$ and $\widetilde{W}_{1}$ has to be zero by Lemma 1.3.16(a). In particular, $p_{1} i j p_{0}=p_{0} i j p_{1}=0$. Thus, we have

$$
\begin{aligned}
& \left(j p_{0} i\right)\left(j p_{0} i\right)=j p_{0} i j\left(1-p_{1}\right) i=j p_{0} i(j i)-j\left(p_{0} i j p_{1}\right) i=j p_{0} i \\
& \left(j p_{1} i\right)\left(j p_{1} i\right)=j p_{1} i j\left(1-p_{0}\right) i=j p_{1} i(j i)-j\left(p_{1} i j p_{0}\right) i=j p_{1} i \\
& j p_{0} i+j p_{1} i=j\left(p_{0}+p_{1}\right) i=j i=1 .
\end{aligned}
$$


This proves $V=j p_{0} i(V) \oplus j p_{1} i(V)$. Moreover, by Lemma 1.3.25(i), $\Theta_{\partial}\left(j p_{0} i(V)\right)$ consists of only $\theta$ since it is a quotient of $\widetilde{W}_{0}$, and $\Theta_{\partial}\left(j p_{1} i(V)\right)$ does not contain $\theta$ since it is a quotient of $\widetilde{W}_{1}$. Applying this process to each of $\theta \in \Theta_{\partial}(V)$ gives the desired decomposition (1.3.27).

The uniqueness of the direct sum decomposition follows from Lemma 1.3.25(b).

Now if $p>0$ and $I R_{\partial}(V)=p^{-1 /(p-1)}$, the decomposition (1.3.27) comes from the decomposition of its $\partial$-Frobenius descendent, via the relation described in Proposition 1.3.18(b). If $p>0$ and $I R_{\partial}(V)>p^{-1 /(p-1)}$, the decomposition (1.3.27) comes from the decomposition of its $\partial$-Frobenius antecedent, via the relation described in Proposition 1.3.18(a).

Now we prove some fundamental properties for tensor products of $\partial$-differential modules with pure $\partial$-radii and pure refined $\partial$-radii. One can combine this with Theorems 1.2.20 and 1.3.26 to obtain corresponding results for general $\partial$-differential modules.

Proposition 1.3.28. Let $V$ and $W$ be two $\partial$-differential modules over $K$ with pure $\partial$-radii $R_{\partial}(V)=R_{\partial}(W)<|u|^{-1}$ and pure refined $\partial$-radii $\theta_{V}$ and $\theta_{W}$, respectively.

(a) Then $W^{\vee}$ has pure refined $\partial$-radii $-\theta_{W}$.

(b) If $\theta_{V}=\theta_{W}$, then we have $R_{\partial}\left(V \otimes W^{\vee}\right)>R_{\partial}(V)$.

(c) If $\theta_{V} \neq \theta_{W}$, then $V \otimes W^{\vee}$ has pure $\partial$-radii $R_{\partial}(V)$ and pure refined $\partial$-radii $\theta_{V}-\theta_{W}$.

(d) Moreover, if we do not assume that $V$ and $W$ have pure refined $\partial$-radii and let $U$ denote the maximal submodule of $V \otimes W^{\vee}$ that has $\partial$-radii strictly larger than $R_{\partial}(V)$, then we have

$$
\operatorname{dim} U=\sum_{\theta \in \kappa_{K^{(s)}}^{\text {alg }}} \operatorname{multi}_{\theta}\left(\Theta_{\partial}(V)\right) \cdot \operatorname{multi}_{\theta}\left(\Theta_{\partial}(W)\right) .
$$

Proof. (a) is straightforward, and (d) follows from (b) and (c) by the decomposition (1.3.27).

When $I R_{\partial}(V)=I R_{\partial}(W)<\omega$, (b) follows from Lemma 1.3.16(c), and (c) follows from the "moreover" part of the same lemma.

When $p>0$ and $I R_{\partial}(V)=I R_{\partial}(W)>p^{-1 /(p-1)}$, (b) and (c) for $V$ and $W$ follow from the same statement for the $\partial$-Frobenius antecedents of $V$ and $W$, by the relation described in Proposition 1.3.18(a).

We now prove (b) and (c) in the case when $p>0$ and $I R_{\partial}(V)=I R_{\partial}(W)=$ $p^{-1 /(p-1)}$. First, Lemma 1.2.18(3) implies that

$$
\varphi_{*}^{(\partial)} V \otimes\left(\varphi_{*}^{(\partial)} W\right)^{\vee}=\left(\varphi_{*}^{(\partial)}\left(V \otimes W^{\vee}\right)\right)^{\oplus p} .
$$


Note that Proposition 1.3.18(2) implies that the multiset of refined intrinsic $\partial$-radii of $V$ is composed of all the solutions to

$$
\left(\frac{x}{p}\right)^{p}-\frac{x}{p}=u \theta_{V}
$$

each with multiplicity $\operatorname{dim} V$, and that the multiset of refined intrinsic $\partial$-radii of $W$ is composed of all the solutions to

$$
\left(\frac{x}{p}\right)^{p}-\frac{x}{p}=u \theta_{W},
$$

each with multiplicity $\operatorname{dim} W$. If $\theta_{V} \neq \theta_{W}$, by (c) in the visible case together with Theorem 1.3.26, the multiset of refined intrinsic $\partial^{\prime}$-radii of $\varphi_{*}^{(\partial)} V \otimes\left(\varphi_{*}^{(\partial)} W\right)^{\vee}$ consists of roots of

$$
\left(\frac{x}{p}\right)^{p}-\frac{x}{p}=u\left(\theta_{V}-\theta_{W}\right),
$$

each with multiplicity $p \operatorname{dim} V \operatorname{dim} W$. Statement (c) then follows from Proposition 1.3.18(b). If $\theta_{V}=\theta_{W}$, by (b) in the visible case together with Theorem 1.3.26, $\varphi_{*}^{(\partial)} V \otimes \varphi_{*}^{(\partial)} W$ has a submodule of dimension $(p-1) \operatorname{dim} V \operatorname{dim} W$ whose intrinsic $\partial^{\prime}$-radius is strictly larger than $p^{-p /(p-1)}$. By Lemma 1.2.18(e), this can happen only if $I R_{\partial}(V \otimes W)>p^{-1 /(p-1)}$, which is what we need to prove in (b).

Remark 1.3.29. We remark that if we do not assume that $\partial$ is of rational type but assume that $R_{\partial}(V)<|\partial|_{K}^{-1}$ instead, all the results in the subsection still hold (note that we do not need Frobenius antecedent in the visible case).

1.4. Multiple derivations. Having studied the situation of one single derivation, we now let multiple commuting derivations interact. This essentially amounts to putting the information from each derivation together. To give the refined radii for multiple derivations a more canonical definition, we will represent the multiset of refined radii as a multiset of differential forms.

Notation 1.4.1. In this subsection, we put $J=\{1, \ldots, m\}$.

Definition 1.4.2. Let $K$ be a differential ring of order $m$, that is, a ring equipped with $m$ commuting derivations $\partial_{1}, \ldots, \partial_{m}$. A $\partial_{J}$-differential module, or simply a differential module, is a finite projective $K$-module $V$ equipped with commuting actions of $\partial_{1}, \ldots, \partial_{m}$. We will apply the results in previous subsections to each $\partial_{j}$ separately.

Definition 1.4.3. Let $K$ and $V$ be as above, and let $R$ be a complete $K$-algebra. For $v \in V$ and $T_{1}, \ldots, T_{m} \in R$, we define the $\partial_{J}$-Taylor series to be

$$
\mathbb{}\left(\boldsymbol{v} ; \partial_{J} ; T_{1}, \ldots, T_{m}\right)=\sum_{e_{J}=0}^{\infty} \frac{\partial_{J}^{e_{J}}(\boldsymbol{v})}{\left(e_{J}\right) !} T_{J}^{e_{J}} \in V \otimes_{K} R,
$$


if it converges.

We will need the following tautological lemma in the proof of Theorem 1.4.20.

Lemma 1.4.4. Let $\partial=\alpha_{1} \partial_{1}+\cdots+\alpha_{m} \partial_{m}$ be another derivation, with $\alpha_{1}, \ldots, \alpha_{m}$ in $K$. To simplify the notation, we formally write $\alpha_{j}=\partial\left(u_{j}\right)$ for any $j \in J$ (and one can check that the formula (1.4.5) can be written with no reference to $\left.u_{j}\right)$. Then, for any $x \in V$, we have

$$
\mathbb{T}\left(x ; \partial_{J} ; \mathbb{T}\left(u_{1} ; \partial ; \delta\right)-u_{1}, \ldots, \mathbb{T}\left(u_{m} ; \partial ; \delta\right)-u_{m}\right)=\mathbb{T}(x ; \partial ; \delta),
$$

as formal power series in $V \otimes_{K} K \llbracket \delta \rrbracket$.

Proof. Since (1.4.5) is a tautological statement, we may assume that $K$ is $\mathbb{Z}$-torsion free. It suffices to show that (1.4.5) is true modulo $\delta^{n}$ for any $\partial_{J}$-differential module $V$ and for any $x \in V$, by induction on $n$. This is clear for $n=1$. Assume that we have proved this claim for $n$ and we need to prove it for $n+1$. It suffices to prove the equality

$$
\frac{\partial}{\partial \delta} \mathbb{T}\left(x ; \partial_{J} ; \mathbb{T}\left(u_{1} ; \partial ; \delta\right)-u_{1}, \ldots, \mathbb{T}\left(u_{m} ; \partial ; \delta\right)-u_{m}\right)=\frac{\partial}{\partial \delta} \mathbb{T}(x ; \partial ; \delta)=\mathbb{T}(\partial(x) ; \partial ; \delta)
$$

modulo $\delta^{n}$ (note that the derivation reduces the exponents on $\delta$ by 1 ). We compute the left hand side as follows.

$$
\begin{gathered}
\frac{\partial}{\partial \delta} \mathbb{T}\left(x ; \partial_{J} ; \mathbb{T}\left(u_{1} ; \partial ; \delta\right)-u_{1}, \ldots, \mathbb{T}\left(u_{m} ; \partial ; \delta\right)-u_{m}\right) \\
=\sum_{e_{J}=0}^{\infty} \frac{\partial_{J}^{e_{J}}(x)}{\left(e_{J}\right) !} \frac{\partial}{\partial \delta}\left(\left(\mathbb{T}\left(u_{1} ; \partial ; \delta\right)-u_{1}\right)^{e_{1}} \cdots\left(\mathbb{T}\left(u_{m} ; \partial ; \delta\right)-u_{m}\right)^{e_{m}}\right) \\
=\sum_{e_{J}=0}^{\infty} \frac{\partial_{J}^{e_{J}}(x)}{\left(e_{J}\right) !}\left(\sum_{j \in J} e_{j} \cdot\left(\mathbb{T}\left(u_{1} ; \partial ; \delta\right)-u_{1}\right)^{e_{1}} \cdots\left(\mathbb{T}\left(u_{j} ; \partial ; \delta\right)-u_{j}\right)^{e_{j}-1}\right. \\
\left.\cdots\left(\mathbb{T}\left(u_{m} ; \partial ; \delta\right)-u_{m}\right)^{e_{m}} \cdot \frac{\partial}{\partial \delta} \mathbb{T}\left(u_{j} ; \partial ; \delta\right)\right) \\
=\sum_{j \in J} \sum_{e_{J}=0}^{\infty} \frac{\partial_{J}^{e_{J}}\left(\partial_{j}(x)\right)}{\left(e_{J}\right) !}\left(\left(\mathbb{T}\left(u_{1} ; \partial ; \delta\right)-u_{1}\right)^{e_{1}}\right. \\
\left.\cdots\left(\mathbb{T}\left(u_{m} ; \partial ; \delta\right)-u_{m}\right)^{e_{m}} \cdot \frac{\partial}{\partial \delta} \mathbb{T}\left(u_{j} ; \partial ; \delta\right)\right)
\end{gathered}
$$

By the induction hypothesis, modulo $\delta^{n}$, this is congruent to

$$
\begin{aligned}
\sum_{j \in J} \mathbb{T}\left(\partial_{j}(x) ; \partial ; \delta\right) \cdot \frac{\partial}{\partial \delta} \mathbb{T}\left(u_{j} ; \partial ; \delta\right) & =\sum_{j \in J} \mathbb{T}\left(\partial_{j}(x) ; \partial ; \delta\right) \cdot \mathbb{T}\left(\partial\left(u_{j}\right) ; \partial ; \delta\right) \\
& =\mathbb{T}\left(\sum_{j \in J} \partial_{j}(x) \partial\left(u_{j}\right) ; \partial ; \delta\right)=\mathbb{T}(\partial(x) ; \partial ; \delta) .
\end{aligned}
$$

This finishes the induction and proves the lemma. 
Definition 1.4.6. Let $K$ be a complete nonarchimedean differential field of order $m$ and characteristic zero, and let $V$ be a nonzero $\partial_{J}$-differential module over $K$. Define the intrinsic radius of $V$ to be

$$
I R(V)=\min _{j \in J}\left\{I_{\partial_{j}}(V)\right\}=\min _{j \in J}\left\{\left|\partial_{j}\right|_{\mathrm{sp}, K} /\left|\partial_{j}\right|_{\mathrm{sp}, V}\right\} .
$$

For $j \in J$, we say $\partial_{j}$ is dominant for $V$ if $I R_{\partial_{j}}(V)=I R(V)$. We define the multiset of intrinsic subsidiary radii $\mathfrak{I} \Re(V)=\{I R(V ; 1), \ldots, I R(V ; \operatorname{dim} V)\}$ by collecting and ordering intrinsic radii from the Jordan-Hölder constituents, as in Definition 1.2.3. We again say that $V$ has pure intrinsic radii if $\mathfrak{I} \mathfrak{R}(V)$ is pure as a multiset.

We similarly define the extrinsic radius $E R(V)$ to be the minimum of $R_{\partial_{j}}(V)$ and the multiset of extrinsic subsidiary radii $\mathfrak{E} R(V)=\{E R(V ; 1), \ldots, E R(V ; \operatorname{dim} V)\}$ by collecting and ordering extrinsic radii from the Jordan-Hölder constituents.

Definition 1.4.7. Let $K$ be a complete nonarchimedean differential field of order $m$ and characteristic zero. We say that $K$ is of rational type with respect to a set of parameters $\left\{u_{j}: j \in J\right\}$ if each $\partial_{j}$ is of rational type with respect to $u_{j}$, and $\partial_{i}\left(u_{j}\right)=0$ for $i \neq j$ in $J$.

Hypothesis 1.4.8. For the rest of this subsection, let $K$ be a complete nonarchimedean field of characteristic zero, equipped with commuting derivations $\partial_{J}$ of rational type with respect to parameters $u_{J}$. Let $V$ be a $\partial_{J}$-differential module with pure $\partial_{j}$-radii for each $j \in J$. We assume moreover that $I R(V)<1$.

Notation 1.4.9. For each $j$, put $s_{j}=-\log \left(\omega R_{\partial_{j}}(V)^{-1}\right), \lambda_{j}=\lambda\left(I R_{\partial_{j}}(V)\right)$, and $r_{j}=r\left(I R_{\partial_{j}}(V)\right)$. By Theorem 1.2.20, we have $s_{j} \in \mathbb{Q} \cdot \log \left|K^{\times}\right|$for any $j$.

Definition 1.4.10. By Theorem 1.3.26, we may replace $K$ by a finite tamely ramified extension such that $V$ admits a direct sum decomposition $V=\oplus V_{\theta_{J}}$, where each direct summand $V_{\theta_{J}}$ has pure refined $\partial_{j}$-radii $\theta_{j}$ for any $j \in J$. Define the multiset of refined radii of $V$, denoted by $\Theta(V)$, to be the collection of $\vartheta=\sum_{j \in J} \theta_{j} d u_{j}$ with multiplicity $\operatorname{dim} V_{\theta_{J}}$, where $\vartheta$ is viewed as an element of

$$
\bigoplus_{j \in J} \kappa_{K^{\mathrm{alg}}}^{\left(s_{j}\right)} d u_{j}
$$

The reason that we write the refined radii in the form of differentials will be justified later, in Theorem 1.4.20.

We will also consider cases where the derivations with larger radii of convergence are ignored.

(i) Let $\Phi \Theta(V)$ be the multiset consisting of elements $\sum \theta_{j} d u_{j}$ with multiplicity $\operatorname{dim} V_{\theta_{J}}$, where the sum is taken over those $j$ such that $I R_{\partial_{j}}\left(V_{\theta_{J}}\right)=\operatorname{IR}\left(V_{\theta_{J}}\right)$; this is called the multiset of refined intrinsic radii. Often, we view it as a 
multiset of elements in

$$
\bigoplus_{j \in J} \kappa_{K^{\text {alg }}}^{(\mathfrak{s})} \frac{d u_{j}}{u_{j}} \quad \text { for } \mathfrak{s}=-\log \left(\omega I R(V)^{-1}\right) .
$$

We remark that this definition does not depend on the field extension of $K$ we made earlier.

(ii) Let $\mathscr{E} \Theta(V)$ be the multiset consisting of elements $\sum \theta_{j} d u_{j}$ with multiplicity $\operatorname{dim} V_{\theta_{J}}$, where the sum is only taken over those $j$ such that $R_{\partial_{j}}\left(V_{\theta_{J}}\right)=R\left(V_{\theta_{J}}\right)$. We call it the refined extrinsic radii.

Definition 1.4.11. Let $\left(\boldsymbol{b}_{1}, \ldots, \boldsymbol{b}_{m}\right) \in(0,1]^{m}$. A norm $|\cdot|_{V}$ on $V$ is $\left(\boldsymbol{b}_{1}, \ldots, \boldsymbol{b}_{m}\right)$ good (or simply good if $\boldsymbol{b}_{j}=I R_{\partial_{j}}(V)$ for all $j \in J$ ) if it is $\boldsymbol{b}_{j}$-good with respect to $\partial_{j}$ for all $j \in J$.

Remark 1.4.12. In contrast to the single derivation case, we do not know if a good norm exists in general, unless we assume that $K$ is discretely valued, in which case, Lemma 1.4.14 below gives an affirmative answer. Hypothesis 1.4.13 below may not be necessary for some of the results later in this subsection, as one might get around using some approximation process. Since we will work with complete discrete valuation field in most applications, we restrict ourselves here to this case.

Hypothesis 1.4.13. For the rest of this subsection, we assume that $K$ is discretely valued.

Lemma 1.4.14. Assume that $\boldsymbol{b}_{j} \in\left(0, I R_{\partial_{j}}(V)\right]$ for any $j \in J$, and that $\boldsymbol{b}_{j}<1$ for all $j$ if $p>0$. Then the differential module $V$ admits $a\left(\boldsymbol{b}_{1}, \ldots, \boldsymbol{b}_{m}\right)$-good norm.

Proof. We first remark that if $I R_{\partial_{j}}(V)<1$, Theorem 1.2.20 implies $I R_{\partial_{j}}(V) \in\left|K^{\times}\right|^{\mathbb{Q}}$. To prove the lemma, we may assume $\boldsymbol{b}_{j}=I R_{\partial_{j}}(V)$.

By the same argument as in Lemma 1.3.9 using Frobenius antecedent, it suffices to prove the lemma under the assumption that $\boldsymbol{b}_{j} \leq \omega$ for any $j \in J$. Note that the $\partial_{j}$-Frobenius antecedent is compatible with $\partial_{j^{\prime}}$ for $j^{\prime} \neq j$. Let $K^{\prime}$ be the completion of $K\left(x_{J}\right)$ with respect to the $\mathrm{e}^{-S_{J}}$-Gauss norm, where we set $\partial_{j}\left(x_{j^{\prime}}\right)=0$ for all $j, j^{\prime} \in J$ and $s_{j}=-\log \left(\omega(\boldsymbol{b}|u|)^{-1}\right)$. In particular, $K^{\prime}$ is discretely valued since $\mathrm{e}^{-s_{j}} \in\left|K^{\times}\right|^{\mathbb{Q}}$ for any $j \in J$.

We first show that $V^{\prime}:=V \otimes K^{\prime}$ has a $\left(\boldsymbol{b}_{1}, \ldots, \boldsymbol{b}_{n}\right)$-good norm. For this, it suffices to show that given any norm $|\cdot|_{V^{\prime}}$ with orthonormal basis $\boldsymbol{e}_{1}, \ldots, \boldsymbol{e}_{d}$, the submodule $M^{\prime}$ of $V^{\prime}$ generated by

$$
\left\{x_{J}^{a_{J}} \partial_{J}^{a_{J}} \boldsymbol{e}_{i}: a_{j} \in \mathbb{Z}_{\geq 0} \text { for any } j \in J \text { and } i \in\{1, \ldots, d\}\right\}
$$

over $\mathrm{O}_{K^{\prime}}$ is a finite $\mathscr{O}_{K^{\prime}}$-module; if so, $M^{\prime}$ gives rise to a norm on $V^{\prime}$, under which $\left|\partial_{j}\right| \leq\left|x_{j}\right|=\mathrm{e}^{-s_{j}}$ for all $j$ verify the conditions of $\left(\boldsymbol{b}_{1}, \ldots, \boldsymbol{b}_{n}\right)$-good norm in Definition 1.3.4. To prove that $M^{\prime}$ is a finite $0_{K^{\prime}}$-submodule, it suffices to prove 
that $\left|x_{j}^{n} \partial_{j}^{n}\right|_{V^{\prime}}$ is bounded for each $j$ as $n \rightarrow+\infty$ (we used here the fact that $K^{\prime}$ is discretely valued, otherwise boundness may not imply finiteness). It is then enough to verify this boundness condition for any $K^{\prime}$-norm on $V^{\prime}$. In particular, for each of $\partial_{j}$, we can choose a $\boldsymbol{b}_{j}$-good norm by Lemma 1.3.9, for which $\left|x_{j}^{n} \partial_{j}^{n}\right|_{V^{\prime}} \leq 1$. Thus $M^{\prime}$ is finite over $\mathcal{O}_{K^{\prime}}$ and hence we have a $\left(\boldsymbol{b}_{1}, \ldots, \boldsymbol{b}_{n}\right)$-good norm on $V^{\prime}$.

This norm restricts to a $K$-norm on $V$ satisfying all the norm conditions in Definition 1.3.4. We use the following lattice lemma to show that it admits an orthogonal basis.

Lemma 1.4.15. Let $F$ be a complete discrete valuation field and let $V$ be a finite dimensional vector space, equipped with a norm compatible with $F$. Assume moreover that the valuation group $\log |V-\{0\}|_{V}$ of $V$ is also discrete. Then $V$ admits an orthogonal basis.

Proof. The proof is almost the same as [Kedlaya 2010, Lemma 1.3.7]. For completeness and the convenience of the reader, we reproduce it here.

We use induction on the dimension $n=\operatorname{dim} V$. When $n=1$, the statement is obvious; any nonzero vector forms an orthogonal basis. Now assuming the statement for $n-1$, we will prove it for an $n$-dimensional $F$-normed vector space $\left(V,|\cdot|_{V}\right)$ whose valuation group is discrete. Pick a nonzero vector $v_{1} \in V$ and denote $W=V / F v_{1}$, provided with the quotient norm $|\cdot|_{W}$; this is again $F$-compatible and has discrete valuation group. By the inductive hypothesis, $W$ admits an orthogonal basis $\bar{v}_{2}, \ldots, \bar{v}_{n}$. For $i=2, \ldots, n$, we pick $v_{i} \in V$ that lifts $\bar{v}_{i} \in W$ such that $\left|v_{i}\right|_{V}=\left|\bar{v}_{i}\right|_{W}$ (this is possible because $V$ has discrete valuation group). We claim that $v_{1}, \ldots, v_{n}$ form an orthogonal basis of $V$.

We need to prove that $|v|_{V}=\max _{i}\left\{\left|x_{i} \| v_{i}\right|_{V}\right\}$ for any $v=x_{1} v_{1}+\cdots+x_{n} v_{n} \in V$. It is clear that $|v|_{V}$ is less than or equal to the right hand side; we need to show $|v|_{V} \geq \max _{i}\left\{\left|x_{i}\right|\left|v_{i}\right|_{V}\right\}$. We prove it the following two cases separately.

(i) If the maximum above is achieved by some $i \geq 2$, we have

$|v|_{V} \geq\left|v \bmod F v_{1}\right|_{W}=\left|x_{2} \bar{v}_{2}+\cdots+x_{n} \bar{v}_{n}\right|_{W}$

$$
=\max _{i=2}^{n}\left\{\left|x_{i}\right|\left|\bar{v}_{i}\right|_{W}\right\}=\max _{i=1}^{n}\left\{\left|x_{i}\right|\left|v_{i}\right|_{V}\right\} .
$$

(ii) We have $\left|x_{1}\right|\left|v_{1}\right|>\left|x_{i}\right|\left|v_{i}\right|$ for all $i=2, \ldots, n$. In this case, we have $|v|=\left|x_{1}\right|\left|v_{1}\right|=\max _{i}\left\{\left|x_{i}\right|\left|v_{i}\right|_{V}\right\}$.

This shows that $v_{1}, \ldots, v_{n}$ form an orthogonal basis of $V$ and finishes the proof of the lemma.

Remark 1.4.16. One may hope to find an analog of Example 1.3.20 for $\partial_{J^{-}}$ differential modules. This, however, amounts to carefully choosing the element $x$ in Example 1.3.20 so that the actions of $\partial_{J}$ commutes. For this, we might need to 
restrict the possible intrinsic refined radii to a subset of

$$
\bigoplus_{j \in J} \kappa_{K^{\mathrm{alg}}}^{(\mathfrak{s})} \frac{d u_{j}}{u_{j}}, \quad \text { where } \mathfrak{s}=-\log \left(\omega I R(V)^{-1}\right) .
$$

Unfortunately, we do not know how to identify this subset in general. Proposition 1.4.17 below partly answers this question.

It would be interesting to know, when $p>0$, whether any element in

$$
\bigoplus_{j \in J} \kappa_{K^{\mathrm{alg}}}^{(\mathfrak{s})} \frac{d u_{j}}{u_{j}}
$$

can appear in the multiset of refined intrinsic radii of some differential module. The referee also pointed out that the reduction of $\partial_{j}$ may give rise to a $\mathscr{D}$-module in characteristic $p$. We do not know if this construction is independent of the choice of good norms. But we suspect that this is related to the reduction of some arithmetic D-module when the differential module comes from one.

Proposition 1.4.17. Assume that $I R(V)<\omega$ and that either $p=0$ or $d=\operatorname{rank} V=1$. Let $\mathfrak{s}=-\log \left(\omega I R(V)^{-1}\right)$. Note that the action of $u_{j} \partial_{j}$ on $K$ induces a derivation on $\kappa_{K^{\text {unr }}}^{(\mathfrak{s})}$. If

$$
\vartheta=\sum_{j \in J} \theta_{j} \frac{d u_{j}}{u_{j}} \in \mathscr{I} \Theta(V),
$$

then for $i, j \in J$, we have $u_{i} \partial_{i} \theta_{j}=u_{j} \partial_{j} \theta_{i}$ in $\kappa_{K^{\text {unr }}}^{(\mathfrak{s})}$.

Proof. By possibly replacing $K$ by a finite tamely ramified extension, we reduce to the case when $V$ is irreducible with a good norm given by an orthonormal basis, and when $V$ has pure refined intrinsic radii $\sum_{j \in J} \theta_{j}\left(d u_{j} / u_{j}\right)$. The $u_{j} \partial_{j}$-action with respect to this basis is given by a matrix $N_{j} \in \operatorname{Mat}_{d \times d}\left(\mathfrak{m}_{K}^{(\mathfrak{s})}\right)$. Since $\partial_{i}$ and $\partial_{j}$ commute with each other for any $i, j \in J$, we have

$$
N_{i} N_{j}+u_{i} \partial_{i}\left(N_{j}\right)=N_{j} N_{i}+u_{j} \partial_{j}\left(N_{i}\right) .
$$

Taking the trace of (1.4.18) gives $d \cdot u_{i} \partial_{i} \theta_{j}=d \cdot u_{j} \partial_{j} \theta_{i}$, which yields the proposition because $d$ is invertible in $\kappa_{K}$.

Before proceeding, we need some notation to use in Theorem 1.4.20 below.

Notation 1.4.19. If $p>0$, we can write an integer $n \in \mathbb{N}$ as $n=a_{0}+p a_{1}+\cdots+p^{k} a_{k}$ with $a_{1}, \ldots, a_{k} \in\{0, \ldots, p-1\}$. Put $\sigma_{p}(n)=a_{0}+\cdots+a_{k}$ if $p>0$, and $\sigma_{p}(n)=0$ if $p=0$. It is straightforward to check that $\sigma_{p}\left(n_{1}\right)+\sigma_{p}\left(n_{2}\right) \geq \sigma_{p}\left(n_{1}+n_{2}\right)$ for $n_{1}, n_{2} \in \mathbb{N}$, and that $|n !|=\omega^{n-\sigma_{p}(n)}$ for $n \in \mathbb{N}$.

The following theorem explains how refined radii change when we consider a different set of derivations, and hence justifies the reason we wrote refined radii in the form of differentials in Definition 1.4.10. 
Theorem 1.4.20. Assume that $V$ has pure refined $\partial_{j}$-radii $\theta_{j} \in \kappa_{K^{\text {alg }}}^{\left(s_{j}\right)}$ for any $j \in J$. Let $K^{\prime}$ be a complete discrete valuation field containing $K$. Let $\partial$ be a derivation on $K^{\prime}$, extending the action of $\alpha_{1} \partial_{1}+\cdots+\alpha_{m} \partial_{m}$ on $K$ to $K^{\prime}$, where $\alpha_{1}, \ldots, \alpha_{m} \in K^{\prime}$. In fact, we have $\alpha_{j}=\partial\left(u_{j}\right)$ for any $j \in J$. We assume that $\partial$ is a derivation of rational type on $K^{\prime}$. Set $s=\min _{j \in J}\left\{s_{j}-\log \left|\alpha_{j}\right|\right\}$ and let $J_{0}$ be a subset of $J$ consisting of $j$ for which $s=s_{j}-\log \left|\alpha_{j}\right|$. Assume moreover that $\operatorname{IR}_{j}(V)<1$ if $j \in J_{0}$. Put $\theta=\sum_{j \in J_{0}} \alpha_{j} \theta_{j} \in \kappa_{K^{\prime \text { alg }}}^{(s)}$.

Then $R_{\partial}\left(V \otimes_{K} K^{\prime}\right) \leq \omega \mathrm{e}^{s}$, and the equality is achieved if and only if $\theta \neq 0$ in $\kappa_{K^{\prime a l g}}^{(s)}$. Moreover, when equivalent statement is verified, $V \otimes_{K} K^{\prime}$ has pure $\partial$-radii $\omega \mathrm{e}^{s}$ and pure refined $\partial$-radii $\theta$.

Proof. For $j \in J$, the equality $\alpha_{j}=\partial\left(u_{j}\right)$ follows from applying $\partial$ to $u_{j}$.

By Lemma 1.4.14 and by possibly enlarging $K$ and $K^{\prime}$, we may assume that $V$ admits a norm given by some orthonormal basis $\underline{\boldsymbol{e}}$ such that, for any $j \in J$,

(i) if $I R_{j}(V)<1$, the norm is good with respect to $\partial_{j}$, and

(ii) if $I R_{j}(V)=1$, the norm is $\boldsymbol{b}_{j}$-good with respect to $\partial_{j}$ for some $\boldsymbol{b}_{j}$ in

$$
\left(\left|\alpha_{j}\right| e^{s-s_{j}}, 1\right) \cap\left|K^{\times}\right|^{\mathbb{Q}} \text {. }
$$

In this case, instead of taking the usual definitions of $r_{j}, \lambda_{j}$, and $s_{j}$, we set $r_{j}=r\left(\boldsymbol{b}_{j}\right), \lambda_{j}=\lambda\left(\boldsymbol{b}_{j}\right)$, and $s_{j}=s-\log \left(\boldsymbol{b}_{j}\left|\alpha_{j}\right|^{-1}\right)$. Note that $s_{j}-\log \left|\alpha_{j}\right|>s$ still holds.

Similarly to Notation 1.3.3, we define integers $r$ and $\lambda$ as follows.

(x) When $|\partial|_{K^{\prime}} \omega \mathrm{e}^{s}<\omega$ we denote $\lambda=0$ and $r=1$.

(xx) When $|\partial|_{K^{\prime}} \omega \mathrm{e}^{s} \in[\omega, 1)$ and $p>0$, let $\lambda$ denote the unique nonnegative integer such that

$$
|\partial|_{K^{\prime}} \omega \mathrm{e}^{s} \in\left[p^{-1 / p^{\lambda-1}(p-1)}, p^{-1 / p^{\lambda}(p-1)}\right),
$$

and put $r=p^{\lambda}$. In this case, we have $\left(|\partial|_{K^{\prime}} \omega \mathrm{e}^{s}\right)^{p^{k}} \leq \omega$ for $k<\lambda$ and hence $\left(|\partial|_{K^{\prime}} \omega \mathrm{e}^{s}\right)^{i} \leq \omega^{\sigma_{p}(i)}$ for $i=1, \ldots, r-1$.

For each $j \in J$, we have

$$
\left|\frac{\partial_{j}^{i}}{i !}\right|_{V} \leq\left|\partial_{j}\right|_{K}^{i} \text {, for } i=1, \ldots, r_{j}-1, \quad \text { and }\left|\partial_{j}^{r_{j}}\right|_{V} \leq\left|u_{j}\right|^{-r_{j}} \mathrm{e}^{-r_{j} s_{j}} \text {. }
$$

For $i=1, \ldots, r$, the action of $\partial^{i}$ on an element $x$ of $\underline{e}$ can be expressed in terms of the actions of $\partial_{J}$, according to the coefficients of $\delta^{i}$ on the left hand side of (1.4.5), applied to $x$. More precisely, for any $j \in J$ and any $i \in \mathbb{N}$, the coefficient of $\delta^{i}$ in $\mathbb{} \mathbb{}\left(u_{j} ; \partial ; \delta\right)-u_{j}$ has norm less than or equal to $\left|\partial\left(u_{j}\right)\right||\partial|_{K^{\prime}}^{i-1}=\left|\alpha_{j}\right||\partial|_{K^{\prime}}^{i-1}$. For any 
coefficient that arises in the $\partial_{J}$-Taylor series expansion, if we put $e_{j}=c_{j}+d_{j} r_{j}$ with $c_{j} \in\left\{0, \ldots, r_{j}-1\right\}$ and $d_{j} \in \mathbb{Z}_{\geq 0}$ for any $j \in J$, then we have

$$
\begin{aligned}
\left|\frac{\partial_{J}^{e_{J}}(x)}{\left(e_{J}\right) !}\right|_{V} & \leq \prod_{j \in J}\left|\frac{\partial_{j}^{d_{j} r_{j}}}{\left(d_{j} r_{j}\right) !}\right|_{V} \cdot \prod_{j \in J}\left|\frac{\partial_{j}^{c_{j}}(x)}{\left(c_{j}\right) !}\right|_{V} \\
& \leq|x|_{V} \cdot \prod_{j \in J}\left|\partial_{j}\right|_{K}^{c_{j}} \cdot \prod_{j \in J}\left(\mathrm{e}^{-d_{j} r_{j} s_{j}} \omega^{-d_{j} r_{j}+\sigma_{p}\left(d_{j} r_{j}\right)}\right),
\end{aligned}
$$

Putting these two bounds together, we see that if a $\delta^{i}$-term on the left hand side of (1.4.5) arises in a term that includes $\partial_{J}^{e_{J}}(x) /\left(e_{J}\right)$ ! (which particularly implies that $\left.i \geq e_{1}+\cdots+e_{m}\right)$, then its norm is smaller than or equal to

$$
\begin{aligned}
& |x||\partial|_{K^{\prime}}^{i-e_{1}-\cdots-e_{m}} \prod_{j \in J}\left|\alpha_{j}\right|^{e_{j}} \prod_{j \in J}\left|\partial_{j}\right|_{K}^{c_{j}} \cdot \prod_{j \in J}\left(\mathrm{e}^{-d_{j} r_{j} s_{j}} \omega^{-d_{j} r_{j}+\sigma_{p}\left(d_{j} r_{j}\right)}\right) \\
& \quad=|x||\partial|_{K^{\prime}}^{i-e_{1}-\cdots-e_{m}} \prod_{j \in J}\left(\left|\partial_{j}\right|_{K}\left|\alpha_{j}\right|\right)^{c_{j}} \cdot \prod_{j \in J}\left(\left(\left|\alpha_{j}\right|_{K} \mathrm{e}^{-s_{j}}\right)^{d_{j} r_{j}} \omega^{-d_{j} r_{j}+\sigma_{p}\left(d_{j} r_{j}\right)}\right) \\
& \quad \leq|x||\partial|_{K^{\prime}}^{i-e_{1}-\cdots-e_{m}} \prod_{j \in J}|\partial|_{K^{\prime}}^{c_{j}} \cdot \prod_{j \in J}\left(\mathrm{e}^{-d_{j} r_{j} s} \omega^{-d_{j} r_{j}+\sigma_{p}\left(d_{j} r_{j}\right)}\right) \\
& \quad\left(\text { note }|\partial|_{K^{\prime}} \geq\left|\partial\left(u_{j}\right)\right|\left|u_{j}\right|^{-1}=\left|\alpha_{j}\right|\left|\partial_{j}\right|_{K}\right) \\
& \quad \leq|x||\partial|_{K^{\prime}}^{i}\left(|\partial|_{K^{\prime}} \omega \mathrm{e}^{s}\right)^{-\sum_{j} d_{j} r_{j}} \omega^{\sigma_{p}\left(\sum_{j} d_{j} r_{j}\right)} .
\end{aligned}
$$

When $i=1, \ldots, r-1$, the coefficient of this $\delta^{i}$-term has norm less than or equal to $|\partial|_{K^{\prime}}^{i}|x|$ by condition $(\mathrm{xx})$. When $i=r$, this $\delta^{i}$-term has norm less than or equal to $|\partial|_{K^{\prime}}^{r}\left(\left(|\partial|_{K^{\prime}} \omega \mathrm{e}^{s}\right)^{-r} \omega\right)|x|=\omega^{-r+1} \mathrm{e}^{-r s}|x|$; the equality can happen only when $\sum_{j} d_{j} r_{j}=r$ and $\sigma_{p}\left(\sum_{j} d_{j} r_{j}\right)=\sum_{j} \sigma_{p}\left(d_{j} r_{j}\right)$, which together yield $e_{j}=r$ for some $j \in J_{0}$ and $e_{j^{\prime}}=0$ for $j^{\prime} \neq j$. When equality of norms is achieved, the corresponding $\delta^{i}$-term is $\alpha_{j}^{r} \partial_{j}^{r}(x) / r$ ! . Therefore, modulo $\mathfrak{m}_{K^{\prime}}^{(r s)+}$, the matrix of $\partial^{r}$ with respect to $\underline{\boldsymbol{e}}$ is congruent to $\sum_{j \in J_{0}} \alpha_{j}^{r} \partial_{j}^{r}$; this is a sum of matrices with single eigenvalues $\alpha_{j}^{r} \theta_{j}^{r}$ for $j \in J_{0}$ (note that, again, $I R_{\partial_{j}}(V)<1$ for all $j \in J_{0}$ ). By Lemma 1.3.14, we have $R_{\partial}(V) \leq \omega \mathrm{e}^{s}$ and this is an equality if and only if $\sum_{j \in J_{0}} \alpha_{j}^{r} \theta_{j}^{r} \neq 0$ in $\kappa_{K^{\prime a l g}}^{(r s)}$, which is equivalent to $\sum_{j \in J_{0}} \alpha_{j} \theta_{j} \neq 0$ in $\kappa_{K^{\prime a l g}}^{(s)}$; note that $r$ is always 1 or a power of $p$. Moreover, if the equivalent condition is satisfied, $V$ has pure refined $\partial$-radii

$$
\left(\sum_{j \in J_{0}} \theta_{j}^{r} \alpha_{j}^{r}\right)^{1 / r}=\sum_{j \in J_{0}} \theta_{j} \alpha_{j}=\theta \in \kappa_{K^{\prime a l g}}^{(s)} .
$$

Corollary 1.4.21. Let $V$ be a d-differential module over $K$ and let

$$
f=\mathbb{}(\cdot ; \partial ; T): K \rightarrow K \llbracket T / u \rrbracket_{0}
$$


and $f^{*} V$ be as in Lemma 1.2.6(d). For $\eta \in[0,|u|)$, let $F_{\eta}$ denote the completion of $K(T)$ with respect to the $\eta$-Gauss norm.

(a) If $\eta \in\left(0, R_{\partial}(V)\right], f^{*} V \otimes F_{\eta}$ has pure intrinsic $\partial_{T}$-radius 1 ; if $\eta \in\left(R_{\partial}(V),|u|\right)$, $f^{*} V \otimes F_{\eta}$ has (extrinsic) $\partial_{T}$-radius $R_{\partial}(V)$.

(b) When $\eta \in\left(R_{\partial}(V),|u|\right)$, we have $\Theta_{\partial_{T}}\left(f^{*} V \otimes F_{\eta}\right)=\Theta_{\partial}(V)$.

Proof. For any $x \in V, f^{*}(\partial(x))=\partial_{T}\left(f^{*}(x)\right)$. The first statement follows from this immediately, and the second statement follows from Theorem 1.4.20. (When $I R_{\partial}(V)=1$, (b) is void.)

Remark 1.4.22. Similar to Remark 1.3.29, if we do not assume that $\partial_{1}, \ldots, \partial_{n}$ are of rational type (but only commuting), the results from this subsection still hold if, for any $\partial_{j}$ for which the refined $\partial_{j}$-radii are relevant, we have $R_{\partial_{j}}(V) \leq\left|\partial_{j}\right|_{K}^{-1}$.

1.5. One-dimensional variation of refined radii. Having established the results for differential modules over a field, we now study the case of a differential module over a rigid analytic annulus or a rigid analytic disc. It is particularly interesting to study how the multisets of (subsidiary) radii of the differential module with respect to different Gauss norms vary as we change the radii which define the Gauss norm. Kedlaya and the author had proved various results on this in [Kedlaya 2010, Chapter 11; Kedlaya and Xiao 2010, Section 2], essentially stating that the (subsidiary) radii are piecewise log-affine functions in the radii of the annulus. In this subsection, we will characterize how the refined radii change as we change the radii for the Gauss norm, in the case when the functions given by the subsidiary radii are in fact log-affine.

Hypothesis 1.5.1. Throughout this subsection, we assume that $K$ is a complete nonarchimedean field of characteristic zero and residual characteristic $p$. We also assume that $K$ is equipped with derivations $\partial_{1}, \ldots, \partial_{m}$ of rational type with respect to $u_{1}, \ldots, u_{m}$.

Notation 1.5.2. Put $J=\{1, \ldots, m\}$ and $J^{+}=J \cup\{0\}$. For $\eta>0$, let $F_{\eta}$ denote the completion of $K(t)$ under the $\eta$-Gauss norm $|\cdot|_{\eta}$. Set $\partial_{0}=d / d t$ on $K[t]$; it extends by continuity to $F_{\eta}$ and ring of functions on discs or annuli. The derivations $\partial_{J^{+}}$ are of rational type on $F_{\eta}$.

Notation 1.5.3. Fix $j \in J^{+}$and an interval $I \subseteq[0, \infty)$. We say that $I$ is an open interval in $[0, \infty)$ if it is of the form $[0, \beta)$ or $(\alpha, \beta)$, where $0<\alpha<\beta$. Put $\dot{I}=I \backslash\{0\}$. For $M$ a $\partial_{j}$-differential module of rank $d$ over $A_{K}^{1}(I), r \in-\log \dot{I}$, and $i \in\{1, \ldots, d\}$, we put

$f_{i}^{(j)}(M, r)=-\log R_{\partial_{j}}\left(M \otimes F_{\mathrm{e}^{-r}} ; i\right), \quad F_{i}^{(j)}(M, r)=f_{1}^{(j)}(M, r)+\cdots+f_{i}^{(j)}(M, r)$.

Theorem 1.5.4. Fix $j \in J^{+}$and an interval $I \subseteq[0,+\infty)$. Let $M$ be a $\partial_{j}$-differential module of rank $d$ over $A_{K}^{1}(I)$. 
(a) (linearity) For $i=1, \ldots, d$, the functions $f_{i}^{(j)}(M, r)$ and $F_{i}^{(j)}(M, r)$ are continuous. They are piecewise affine on the locus where $f_{i}^{(j)}(M, r)>-\log \left|u_{j}\right|$ if $j \in J$; and they are piecewise affine on all of $-\log \dot{I}$ if $j=0$.

(b) (weak integrality)

(b1) Suppose $p=0$ or $j=0$. If $i=d$ or $f_{i+1}^{(j)}\left(M, r_{0}\right)<f_{i}^{(j)}\left(M, r_{0}\right)$, the slopes of $F_{i}^{(j)}(M, r)$ in some neighborhood of $r=r_{0}$ belong to $\mathbb{Z}$. Consequently, the slopes of each $f_{i}^{(j)}(M, r)$ and $F_{i}^{(j)}(M, r)$ belong to $\frac{1}{1} \mathbb{Z} \cup \cdots \cup \frac{1}{d} \mathbb{Z}$.

(b2) Suppose $p>0$ and $j \in J$. If $f_{i}^{(j)}\left(M, r_{0}\right)>1 /\left(p^{n}(p-1)\right) \log p-\log \left|u_{j}\right|$ for some $n \in \mathbb{Z}_{\geq 0}$, then the slopes of each $f_{i}^{(j)}(M, r)$ and $F_{i}^{(j)}(M, r)$ in some neighborhood of $r_{0}$ belong to $\frac{1}{p^{n} d !} \mathbb{Z}$.

(c) (monotonicity) Suppose $0 \in I$ and suppose either $j \in J$, or $j=0$ and $f_{i}^{(0)}\left(M, r_{0}\right)>r_{0}$. Then the slopes of $F_{i}^{(j)}\left(M, r_{0}\right)$ are nonpositive in a neighborhood of $r_{0}$.

(d) (convexity) For $i=1, \ldots, d$, the function $F_{i}^{(j)}(M, r)$ is convex.

(e) (decomposition) Assume that I is an open interval in $[0,+\infty)$. Suppose that for some $i \in\{1, \ldots, d\}, F_{i}^{(j)}(M, r)$ is affine and $f_{i}^{(j)}(M, r)>f_{i+1}^{(j)}(M, r)$ for $r \in-\log I$. Then we can write $M$ uniquely as the direct sum of two $\partial_{j}$ differential submodules $M_{1}$ and $M_{2}$, such that, for any $\eta \in I$, the multiset of $\partial_{j}$-radii of $M_{1} \otimes F_{\eta}$ exactly consists of the smallest $i$ elements in the multiset of $\partial_{j}$-radii of $M \otimes F_{\eta}$.

Proof. This is [Kedlaya and Xiao 2010, Theorems 2.2.5, 2.2.6, and 2.3.5].

Notation 1.5.5. Let $I \subseteq[0,+\infty)$ be an interval and let $M$ be a $\partial_{J^{+}}$-differential module of rank $d$ on $A_{K}^{1}(I)$. For $r \in-\log \dot{I}$ and $i \in\{1, \ldots, d\}$, we put

$f_{i}(M, r)=-\log I R\left(M \otimes F_{\mathrm{e}^{-r}} ; i\right) \quad$ and $\quad F_{i}(M, r)=f_{1}(M, r)+\cdots+f_{i}(M, r)$.

Suppose that $I \subseteq[0,1)$ and that $\left|u_{j}\right|=1$ for any $j \in J$, we put

$\hat{f}_{i}(M, r)=-\log E R\left(M \otimes F_{\mathrm{e}^{-r}} ; i\right) \quad$ and $\quad \hat{F}_{i}(M, r)=\hat{f}_{1}(M, r)+\cdots+\hat{f}_{i}(M, r)$.

Theorem 1.5.6. Fix an interval $I \subseteq[0,+\infty)$. Let $M$ be a $\partial_{J^{+-}}$differential module of rank d over $A_{K}^{1}(I)$.

(a) (linearity) For $i=1, \ldots, d$, the functions $f_{i}(M, r)$ and $F_{i}(M, r)$ are continuous and piecewise affine.

(b) (integrality) If $i=d$ or $f_{i}\left(M, r_{0}\right)>f_{i+1}\left(M, r_{0}\right)$, then the slopes of $F_{i}(M, r)$ in some neighborhood of $r_{0}$ belong to $\mathbb{Z}$. Consequently, the slopes of each $f_{i}(M, r)$ and $F_{i}(M, r)$ belong to $\frac{1}{1} \mathbb{Z} \cup \cdots \cup \frac{1}{d} \mathbb{Z}$.

(c) (monotonicity) Suppose that $0 \in I$. Then the slopes of $F_{i}(M, r)$ are nonpositive, and each $F_{i}(M, r)$ is constant for $r$ sufficiently large. 
(d) (convexity) For $i=1, \ldots, d$, the function $F_{i}(M, r)$ is convex.

(e) (decomposition) Suppose that I is an open interval in $(0,+\infty)$, and suppose that, for some $i \in\{1, \ldots, d-1\}$, the function $F_{i}(M, r)$ is affine and $f_{i}(M, r)>$ $f_{i+1}(M, r)$ for $r \in-\log \dot{I}$. Then $M$ can be uniquely written as the direct sum of two $\partial_{J^{+}}$-differential submodules $M_{1}$ and $M_{2}$ such that, for any $\eta \in \dot{I}$, the multiset of intrinsic radii of $M_{1} \otimes F_{\eta}$ exactly consists of the smallest $i$ elements in the multiset of intrinsic radii of $M \otimes F_{\eta}$.

(f) (dichotomy) Suppose that I is an open interval in $[0,+\infty)$ and that $M$ is not the direct sum of two nonzero $\partial_{J^{+}}$-differential submodules. If $f_{1}(M, r)$ is affine for $r \in-\log \dot{I}$, then, for each $j \in J^{+}$,

(1) either $M \otimes F_{\eta}$ has pure intrinsic $\partial_{j}$-radii and the intrinsic $\partial$-radius equals I $R\left(M \otimes F_{\eta}\right)$ for all $\eta \in \dot{I}$, or

(2) we have $I R_{\partial_{j}}\left(M \otimes F_{\eta}\right)>I R\left(M \otimes F_{\eta}\right)$ for all $\eta \in \dot{I}$.

Moreover, if $\left|u_{j}\right|=1$ for any $j \in J$ and if $I \subseteq[0,1)$, then the same statements above except $(c)$ hold for $\hat{f}_{i}(M, r)$ and $\hat{F}_{i}(M, r)$ in place of $f_{i}(M, r)$ and $F_{i}(M, r)$, respectively. In this case, the following statement holds.

$\left(\mathrm{c}^{\prime}\right)$ (monotonicity) Suppose that $0 \in I$. For $i=1, \ldots, d$, for any point $r_{0}$ where $\hat{f}_{i}\left(M, r_{0}\right)>r_{0}$, the slopes of $\hat{F}_{i}(M, r)$ are nonpositive in some neighborhood of $r_{0}$. We also have $\hat{f}_{i}(M, r)=r$ for $r$ sufficiently large.

Proof. Statements (a)-(e) for $f_{i}(M, r)$ and $F_{i}(M, r)$ are proved in [Kedlaya and Xiao 2010, Theorems 2.4.4 and 2.5.1]. Statements (a), (b), (c'), (d), and (e) for $\hat{f}_{i}(M, r)$ and $\hat{F}_{i}(M, r)$ can be proved similarly as follows.

Let $\widetilde{K}$ denote the completion of $K\left(x_{J}\right)$ with respect to the $(1, \ldots, 1)$-Gauss norm. For $I=[\alpha, \beta) \subseteq[0,1)$, the Taylor series defines an injective continuous homomorphism $\left.\left.\tilde{f}^{*}: K\langle\alpha / t, t / \beta\}\right\} \rightarrow \widetilde{K}\{\alpha / t, t / \beta\}\right\}$ such that $\tilde{f}^{*}\left(u_{j}\right)=u_{j}+x_{j} t$ (as in [Kedlaya and Xiao 2010, Notation 2.4.1]). For $\eta \in(\alpha, \beta)$, we use $\widetilde{F}_{\eta}$ to denote the completion of $\widetilde{K}(t)$ with respect to the $\eta$-Gauss norm. Then $\tilde{f}^{*}$ extends to an injective isometric homomorphism $\tilde{f}^{*}: F_{\eta} \hookrightarrow \widetilde{F}_{\eta}$.

We view $\tilde{f}^{*} M$ as a $\partial_{0}$-differential module on $A_{\widetilde{K}}^{1}[\alpha, \beta)$. Since

we have

$$
\left.\partial_{0}\right|_{\tilde{f}^{*} M}=\left.\partial_{0}\right|_{M}+\left.\sum_{j \in J} x_{j} \partial_{j}\right|_{M},
$$

$$
R_{\partial_{0}}\left(M \otimes \widetilde{F}_{\eta}\right)=\min _{j \in J^{+}}\left\{R_{\partial_{j}}\left(M \otimes F_{\eta}\right)\right\}=E R\left(M \otimes F_{\eta}\right), \text { for any } \eta \in[\alpha, \beta) .
$$

In other words, $f_{i}^{(0)}\left(\tilde{f}^{*} M, r\right)=\hat{f}_{i}(M, r)$ for $r \in(-\log \beta,-\log \alpha)$. The theorem follows from Theorem 1.5.4; to obtain the decomposition in (e), we use Lemma 1.1.10 and Remark 1.1.11 to glue the decompositions over $A_{\widetilde{K}}^{1}[\alpha, \beta)$ and over $F_{\eta}$ for some $\eta \in(\alpha, \beta)$. 
We now prove (f) for the intrinsic radii; the proof for the extrinsic radii is similar.

Fix $j \in J^{+}$. Assume that we are not in case (2). Then $I R_{\partial_{j}}\left(M \otimes F_{\eta}\right)=\operatorname{IR}\left(M \otimes F_{\eta}\right)$ for some $\eta \in \dot{I}$. By Theorem 1.5.4(d), the that that $f_{1}^{(j)}(M, r)$ is convex forces $I R_{\partial_{j}}\left(M \otimes F_{\eta}\right)=I R\left(M \otimes F_{\eta}\right)$ for all $\eta \in \dot{I}$. Now, if $I R_{\partial_{j}}\left(M \otimes F_{\eta} ; 2\right)>I R\left(M \otimes F_{\eta}\right)$ for all $\eta \in(\alpha, \beta)$, the decomposition (e) would imply that $M$ is decomposable, which contradicts the assumption. Therefore, we have $I R_{\partial_{j}}\left(M \otimes F_{\eta} ; 2\right)=\operatorname{IR}\left(M \otimes F_{\eta}\right)$ for some $\eta \in \dot{I}$. By Theorem 1.5.4(d) again, we have the equality for all $\eta \in \dot{I}$. Continuing this argument for the third smallest and other subsidiary $\partial_{j}$-radii leads us to case (1).

Next, we discuss how the multiset of refined $\partial_{j}$-radii of the $\partial_{j}$-differential module $M$ changes when we base change the $\partial_{j}$-differential module $M$ to the completions with respect to different Gauss norms, in the case when

$$
f_{1}^{(j)}(M, r)=\cdots=f_{\operatorname{rank} M}^{(j)}(M, r)
$$

is affine. Before proving general results, we first look at an example of $\partial_{j}$-differential module with pure refined $\partial_{j}$-radii when base changed to any completion with respect to the Gauss norm. It is a 1-dimensional family analog of Example 1.3.20.

Example 1.5.7. Let $j \in J^{+}$and let $(\alpha, \beta) \subseteq(0, \infty)$ be an open interval. Fix $b \in \mathbb{Q}$ and $\theta \in \kappa_{K^{\text {alg }}}^{(a)}$, where $a \in-\log \left|K^{\times}\right|^{\mathbb{Q}}$. Assume that

$$
\mathrm{e}^{a} \alpha^{b}, \mathrm{e}^{a} \beta^{b}< \begin{cases}1 & \text { if } p=0 \\ p^{1 / p} & \text { if } p>0\end{cases}
$$

We will see that this includes some nonvisible radii. As noted in Remark 1.3.21, we cannot loosen the restriction in (1.5.8) from $p^{1 / p}$ to $p^{1 /(p-1)}$.

Let $e$ be the prime-to- $p$ part of the denominator of $b$. We have the following:

(i) If $p=0$, then $a \in-\log \left|\left(K^{\prime}\right)^{\times}\right|$and $\theta \in \kappa_{K^{\prime}}^{(a)}$ for some finite tamely ramified extension $K^{\prime} / K$. Let $x \in \mathfrak{m}_{K^{\prime}}^{(a)}$ be a lift of $\theta$. We set $n=0$ and $d=1$ in this case.

(ii) If $p>0$ and $j=0$, there exists $n \in \mathbb{N}$ such that

$$
\theta^{p^{n}} \in \kappa_{K^{\prime}}^{\left(p^{n} a\right)} \quad \text { with } p^{n} a \in-\log \left|\left(K^{\prime}\right)^{\times}\right| \text {and } p^{n} e b \in p \mathbb{Z},
$$

for some finite tamely ramified extension $K^{\prime} / K$. Let $x \in \mathfrak{m}_{K^{\prime}}^{\left(p^{n} a\right)}$ be a lift of $\theta^{p^{n}}$. We set $d=p^{n}$.

(ii') If $p>0$ and $j \in J$, there exists $n \in \mathbb{N}$ such that $\theta^{p^{n}} \in\left(\kappa_{K^{\prime}}^{\left(p^{n-1} a\right)}\right)^{p}$ and $p^{n} e b \in \mathbb{Z}$ with $p^{n-1} a \in-\log \left|\left(K^{\prime}\right)^{\times}\right|$for some finite tamely ramified extension $K^{\prime} / K$. Let $x \in \mathfrak{m}_{K^{\prime\left(\partial_{j}\right)}}^{\left(p^{n} a\right)}$ be a lift of $\theta^{p^{n}}$; this is possible by Lemma 1.2.16. 
Let $A_{K^{\prime}}^{1}\left(\alpha^{1 / e}, \beta^{1 / e}\right)$ be the open annulus with coordinate $t^{1 / e}$. Let $\mathscr{L}_{x, b,(n)}^{(j)}$ denote the $\partial_{j}$-differential module over $A_{K^{\prime}}^{1}\left(\alpha^{1 / e}, \beta^{1 / e}\right)$ of rank $d$ with basis $\left\{\boldsymbol{e}_{1}, \ldots, \boldsymbol{e}_{d}\right\}$, on which $\partial_{j}$ acts per description

$$
\partial_{j} \boldsymbol{e}_{i}=\boldsymbol{e}_{i+1} \text { for } i=1, \ldots, d-1 \quad \text { and } \partial_{j} \boldsymbol{e}_{d}= \begin{cases}x t^{-d b} u_{j}^{-d} \boldsymbol{e}_{1} & \text { if } j \in J \\ x t^{-d(b+1)} \boldsymbol{e}_{1} & \text { if } j=0\end{cases}
$$

We added $u_{j}^{-d}$ and $t^{-d}$ in the definition to balance the different normalizations on intrinsic $\partial_{j}$-radii.

Lemma 1.5.9. Keep the notation as in Example 1.5.7. If we set $F_{\mathrm{e}^{-r}}^{\prime}=F_{\mathrm{e}^{-r}}\left(t^{1 / e}\right)$, then for any $r \in(-\log \beta,-\log \alpha), \mathscr{L}_{x, b,(n)}^{(j)} \otimes F_{\mathrm{e}^{-r}}^{\prime}$ has pure intrinsic $\partial_{j}$-radii $\omega \mathrm{e}^{a-b r}$ and pure refined $\partial_{j}$-radii $\theta t^{-b}$.

Proof. Comparing this with Example 1.3.20 shows that for any $r, \mathscr{L}_{x, b,(n)}^{(j)} \otimes F_{\mathrm{e}^{-r}}^{\prime}$ is isomorphic to $\mathscr{L}_{x t^{-d b} u_{j}^{-d},(n)}$ if $j \in J$, and to $\mathscr{L}_{x t^{-d(b+1)},(n)}$ if $j=0$. Applying Lemma 1.3.23 to this $\partial_{j}$-differential module yields the result; note that the condition (1.5.8) corresponds to the condition on $\mathfrak{s}$ in Example 1.3.20.

Theorem 1.5.10. Fix $j \in J^{+}$. Let $M$ be a $\partial_{j}$-differential module over an open annulus $A_{K}^{1}(\alpha, \beta)$ such that $M \otimes F_{\mathrm{e}^{-r}}$ has pure intrinsic $\partial_{j}$-radii $\omega \mathrm{e}^{a-b r}<1$ for any $r \in(-\log \beta,-\log \alpha)$ (this implies that $f_{1}^{(j)}(M, r)=\cdots=f_{\operatorname{dim} M}^{(j)}(M, r)$ is an affine function with slope $b)$. Let e be the prime-to-p part of the denominator of $b$. Then there exists a unique direct sum decomposition

$$
M=\bigoplus_{\left\{\mu_{e} \theta\right\} \subseteq \kappa_{K^{\text {alg }}}^{(a)}} M_{\left\{\mu_{e} \theta\right\}}
$$

of $\partial_{j}$-differential modules over $A_{K}^{1}(\alpha, \beta)$ where the sum is over all $\mu_{e} \rtimes \operatorname{Gal}\left(K^{\mathrm{alg}} / K\right)$ orbits of $\kappa_{K^{\text {alg }}}^{(a)}$, and the refined $\partial_{j}$-radii of $M_{\left\{\mu_{e} \theta\right\}} \otimes F_{\eta}$ for any $\eta \in(\alpha, \beta)$ is a multiset consisting of the $\mu_{e} \rtimes \mathrm{Gal}\left(K^{\mathrm{alg}} / K\right)$-orbits $\left\{t^{-b} \theta\right\}$ with appropriate multiplicities.

Moreover, if $K^{\prime}$ is a finite tamely ramified tension of $K$ such that all the $\theta$ in the above decomposition belong to $\bigcup_{n}\left(\kappa_{K^{\prime}}^{\left(p^{n} s\right)}\right)^{1 / p^{n}}$, then we have a unique direct sum decomposition

$$
M \otimes_{K\{\{\alpha / t, t / \beta\}\}} K^{\prime}\left\{\left\{\alpha^{1 / e} / t^{1 / e}, t^{1 / e} / \beta^{1 / e}\right\}\right\}=\bigoplus_{\theta \in \kappa_{K^{(a)}}^{\text {alg }}} M_{\theta}
$$

of $\partial_{j}$-differential modules over $A_{K^{\prime}}^{1}\left(\alpha^{1 / e}, \beta^{1 / e}\right)$ such that $M_{\theta} \otimes K^{\prime} F_{\eta}^{\prime}$ has pure refined $\partial_{j}$-radii $t^{-b} \theta$ for any $\eta \in(\alpha, \beta)$.

Proof. First of all, since defining a $\partial_{j}$-differential module only needs finite data, we may assume that $\mathbb{Q} \cdot \log \left|K^{\times}\right| \neq \mathbb{R}$. 
The decomposition as stated in the theorem, if it exists, is determined by the decomposition of $M \otimes F_{\mathrm{e}^{-r}}$ for each $r \in(-\log \beta,-\log \alpha)$; it is hence unique. We may always replace $M$ by $M \otimes_{K\{\{\alpha / t, t / \beta\}\}} K^{\prime}\left\{\left\{\alpha^{1 / e} / t^{1 / e}, t^{1 / e} / \beta^{1 / e}\right\}\right\}$ for $e$ and any finite tamely ramified extension $K^{\prime}$ of $K$, and we may recover the result for $M$ using Galois descent. In particular, we may assume that $e=1$. Moreover, using Lemma 1.1.10 and Remark 1.1.11, it suffices to first obtain the decomposition in a neighborhood of each radius in $(\alpha, \beta)$ and then glue the decompositions on overlaps.

Let $r_{0} \in(-\log \beta,-\log \alpha)$ be a point. We first assume that $I R_{\partial_{j}}\left(M \otimes F_{\mathrm{e}^{-r_{0}}}\right)<1$ when $p=0$, and $I R_{\partial_{j}}\left(M \otimes F_{\mathrm{e}^{-r_{0}}}\right)<p^{-1 / p(p-1)}$ when $p>0$ (note that this restriction still allows some nonvisible radii). By shrinking the interval $(\alpha, \beta)$ to a smaller neighborhood of $r_{0}$, we may assume that the condition above at $r_{0}$ holds for all points in $(-\log \beta,-\log \alpha)$. Pick a point $r_{1} \in(-\log \beta,-\log \alpha)$ which does not belong to $\mathbb{Q} \cdot \log \left|K^{\times}\right|$.

Let $\theta t^{-b} \in \mathscr{I} \Theta_{\partial_{j}}\left(M \otimes F_{\mathrm{e}^{-r_{1}}}\right)$ be an element in the multiset of refined intrinsic $\partial_{j}$-radii, with multiplicity $\mu$. Since $M \otimes F_{\mathrm{e}^{-r_{1}}}$ has pure intrinsic $\partial_{j}$-radii $\omega \mathrm{e}^{a-b r_{1}}$, we have

$$
\theta t^{-b} \in \kappa_{F_{\mathrm{e}^{-r_{1}}}^{\text {alg }}}^{\left(a-b r_{1}\right)} \cong t^{-b} \kappa_{K^{\text {alg }}}^{(a)}
$$

here the latter isomorphism follows from our choice $r_{1} \notin \mathbb{Q} \cdot \log \left|K^{\times}\right|$. We may replace $K$ by a finite tamely ramified extension so that

$$
\theta \in \bigcup_{n}\left(\kappa_{K}^{\left(p^{n} a\right)}\right)^{1 / p^{n}}
$$

The construction in Example 1.5 .7 gives a $\partial_{j}$-differential module $\mathscr{L}_{x, b,(n)}^{(j)}$ over $A_{K}^{1}(\alpha, \beta)$ such that $\mathscr{L}_{x, b,(n)}^{(j)} \otimes F_{\mathrm{e}^{-r}}$ has pure $\partial_{j}$-radii $\omega \mathrm{e}^{a-b r}$ and pure intrinsic $\partial_{j}$-radii $\theta t^{-b}$ for any $r \in(-\log \beta,-\log \alpha)$.

If we set $N=M \otimes\left(\mathscr{L}_{x, b,(n)}^{(j)}\right)^{\vee}$, then we have $I R_{\partial_{j}}\left(N \otimes F_{\mathrm{e}^{-r}}\right) \leq \omega \mathrm{e}^{a-b r}$ for any $r \in(-\log \beta,-\log \alpha)$. Moreover, Proposition 1.3.19 and Theorem 1.3.26 together imply that

$$
f_{1}^{(j)}\left(M, r_{1}\right)=f_{1}^{(j)}\left(N, r_{1}\right)=f_{(\operatorname{dim} M-\mu) d}^{(j)}\left(N, r_{1}\right)>f_{(\operatorname{dim} M-\mu) d+1}^{(j)}\left(N, r_{1}\right) .
$$

By Theorem 1.5.6(d), the same inequality holds for all $r \in(-\log \beta,-\log \alpha)$ in place of $r_{1}$ because a convex function below a linear function is same as the linear function if and only if the two functions touch at some point. By Theorem 1.5.4(e), we have a unique decomposition of $\partial_{j}$-differential modules $N=N_{0} \oplus N_{1}$ such that, for any $r \in(-\log \beta,-\log \alpha), N_{0} \otimes F_{\mathrm{e}^{-r}}$ has pure intrinsic $\partial_{j}$-radii $\omega \mathrm{e}^{a-b r}$ and $I R_{\partial_{j}}\left(N_{1} \otimes F_{\mathrm{e}^{-r}}\right)>\omega \mathrm{e}^{a-b r}$. By the same argument as in Theorem 1.3.26, this implies that $M$ admits a decomposition of $\partial_{j}$-differential modules $M=M_{\theta} \oplus M^{\prime}$ over $A_{K}^{1}(\alpha, \beta)$ such that $M_{\theta} \otimes\left(\mathscr{L}_{x, b,(n)}^{(j)}\right)^{\vee}=N_{1}$ and $M^{\prime} \otimes\left(\mathscr{L}_{x, b,(n)}^{(j)}\right)^{\vee}=N_{0}$. By 
Proposition 1.3.28 and Lemma 1.5.9, for any $r \in(-\log \beta,-\log \alpha), M_{\theta} \otimes F_{\mathrm{e}^{-r}}$ has pure refined intrinsic $\partial_{j}$-radii $\theta t^{-b}$, and the multiset of refined intrinsic $\partial_{j}$-radii of $M^{\prime} \otimes F_{\mathrm{e}^{-r}}$ does not contain $\theta t^{-b}$. We obtain the decomposition in the theorem by applying this argument to every $\theta$.

To finish the proof, it suffices to consider the case when $p>0$ and

$$
I R_{\partial_{j}}\left(M \otimes F_{\mathrm{e}^{-r}}\right) \in\left[p^{-1 / p(p-1)}, 1\right) .
$$

But in this case, the $\partial_{j}$-Frobenius antecedent of $M$ exists over the annulus with radii in a neighborhood of $r$. The decomposition follows from the decomposition of the $\partial_{j}$-Frobenius antecedents of $M$ (applied iteratively until the intrinsic $\partial_{j}$-radii fall in the range above).

Remark 1.5.11. The artificial reduction to the case $\mathbb{Q} \cdot \log \left|K^{\times}\right| \neq \mathbb{R}$ is to deduce $\theta \in \kappa_{K^{\text {alg }}}^{(a)}$. This fact can also be proved using Newton polygons if the $f_{1}^{(j)}(M, r)$ is not constantly $p^{-1 /(p-1)}$, in which case one may alternatively use the Frobenius pushforward to reduce to the visible case.

Theorem 1.5.12. Let $I$ be an open interval of $[0,+\infty)$ and let $M$ be a $\partial_{J^{+-}}$ differential module over $A_{K}^{1}(I)$ such that $M \otimes F_{\mathrm{e}^{-r}}$ has pure intrinsic radii $\omega \mathrm{e}^{a-b r}<1$ for $r \in-\log (\dot{I})$. Let e denote the prime-to-p part of the denominator of $b$. Then there exists a unique direct sum decomposition $M=\bigoplus_{\left\{\mu_{e} \vartheta\right\}} M_{\left\{\mu_{e} \vartheta\right\}}$ of $\partial_{J^{+}-\text {differential }}$ modules over $A_{K}^{1}(I)$, where the sum is taken over all $\mu_{e} \rtimes \operatorname{Gal}\left(K^{\mathrm{alg}} / K\right)$-orbits of

$$
\bigoplus_{j \in J} \kappa_{K^{\mathrm{alg}}}^{(a)} \frac{d u_{j}}{u_{j}} \oplus \kappa_{K^{\mathrm{alg}}}^{(a)} \frac{d t}{t},
$$

and the refined intrinsic radii of $M_{\left\{\mu_{e} \vartheta\right\}} \otimes F_{\eta}$ for any $\eta \in-\log \dot{I}$ is a multiset consisting of the $\mu_{e} \rtimes \operatorname{Gal}\left(K^{\text {alg }} / K\right)$-orbits $\left\{t^{-b} \vartheta\right\}$ with appropriate multiplicities.

Moreover, there exists a finite tamely ramified tension $K^{\prime}$ of $K$ such that we have a unique direct sum decomposition

$$
M \otimes_{K[t]} K^{\prime}\left[t^{1 / e}\right]=\bigoplus_{\vartheta \in \bigoplus_{j \in J} \kappa_{K^{\text {alg }}}^{(a)} \frac{d u_{j}}{u_{j}} \bigoplus \kappa_{K^{\text {alg }}}^{(a)} \frac{d t}{t}} M_{\vartheta}
$$

of $\partial_{J^{+}}$-differential modules over $A_{K^{\prime}}^{1}\left(I^{1 / e}\right)$ such that $M_{\vartheta} \otimes K^{\prime} F_{\eta}^{\prime}$ has pure refined intrinsic radii $t^{-b} \vartheta$ for any $\eta \in-\log \dot{I}$.

Proof. We first treat the case when $0 \notin I$. Without loss of generality, we assume that $M$ is not a direct sum of two nonzero sub- $\partial_{J^{+}}$-modules, which implies the dichotomy given by Theorem 1.5.6(f). We may apply Theorem 1.5.10 to the $\partial_{j}$ for which case (f1) of Theorem 1.5.6 holds for $M$ and note that the decompositions for different $\partial_{j}$ given by Theorem 1.5 .10 are compatible. This gives rise to the desired decomposition. 
Now, we consider the case when $I=[0, \beta)$. Since we have already proved the theorem over $(\alpha, \beta)$ for any $\alpha>0$, it suffices to find the decomposition for $I=[0, \alpha)$ for some $\alpha \in(0,1)$. Note that when $\alpha$ is sufficiently small, $M \otimes A_{K}^{1}[0, \alpha)$ is trivial as a $\partial_{0}$-differential module and hence is the pullback of a $\partial_{J}$-differential module $M_{0}$ over $K$ along the natural morphism $K \rightarrow K\{\{t / \alpha\}\}$. The decomposition (1.5.13) follows from the decomposition of $M_{0}$ given by Theorem 1.3.26.

We have a similar result for refined extrinsic radii, but only over $A_{K}^{1}(I)$; this is because adjoining $t^{1 / e}$ would change the extrinsic radii. This subtlety also comes up when considering differential modules over discs (as opposed to annuli) and trying to extend the decomposition into the center of the disc: this is only possible if the functions defined by the extrinsic radii are "constant".

Theorem 1.5.14. Assume that $\left|u_{j}\right|=1$ for all $j \in J$. Let $M$ be a $\partial_{J^{+}}$-differential module over an open annulus $A_{K}^{1}(I)$ with $I \subseteq(0,1)$. Assume that $M \otimes F_{\mathrm{e}^{-r}}$ has pure extrinsic radii $\omega \mathrm{e}^{a-b r}<\mathrm{e}^{-r}$ for $r \in-\log (\dot{I})$. Let e denote the prime-to-p part of the denominator of $b$. Then there exists a unique direct sum decomposition

$$
M=\bigoplus_{\left\{\mu_{e} \hat{\vartheta}\right\}} M_{\left\{\mu_{e} \hat{\vartheta}\right\}}
$$

of $\partial_{J^{+}}$-differential modules over $A_{K}^{1}(I)$, where the direct sum is taken over all $\mu_{e} \rtimes \operatorname{Gal}\left(K^{\mathrm{alg}} / K\right)$-orbits

$$
\left\{\mu_{e} \hat{\vartheta}\right\} \quad \text { in } \bigoplus_{j \in J} \kappa_{K^{\text {alg }}}^{(a)} d u_{j} \oplus \kappa_{K^{\text {alg }}}^{(a)} d t
$$

and the multiset of refined extrinsic radii of $M_{\left\{\mu_{e} \hat{\vartheta}\right\}} \otimes F_{\eta}$ exactly consists of the $\mu_{e} \rtimes \operatorname{Gal}\left(K^{\mathrm{alg}} / K\right)$-orbits $\left\{t^{-b} \mu_{e} \hat{\vartheta}\right\}$ with appropriate multiplicities, for any $\eta \in \dot{I}$.

Proof. The proof is the same as Theorem 1.5.12.

Proposition 1.5.16. Fix $j \in J^{+}$. Let $M$ be a $\partial_{j}$-differential module over an open disc $A_{K}^{1}[0, \alpha)$ such that $M \otimes F_{\eta}$ for any $\eta$ in a neighborhood of $\eta=\alpha$ has pure $\partial_{j}$-radii $\omega \mathrm{e}^{s}$, where $\omega \mathrm{e}^{s}$ is independent of $\eta$, and is strictly less than $\left|u_{j}\right|$ if $j \in J$ and less than $\alpha$ if $j=0$. Then there exists a unique direct sum decomposition $M=\bigoplus_{\{\theta\} \subset \kappa_{K}^{(s)}} M_{\{\theta\}}$ of $\partial_{j}$-differential modules over $A_{K}^{1}[0, \alpha)$, where the direct sum is taken over all $\mathrm{Gal}\left(K^{\mathrm{alg}} / K\right)$-orbits $\{\theta\}$ of $\kappa_{K}^{(s)}$, and the multiset of refined $\partial_{j}$-radii of $M_{\{\theta\}} \otimes F_{\eta}$ consists of the $\operatorname{Gal}\left(K^{\mathrm{alg}} / K\right)$-orbits $\{\theta\}$ with appropriate multiplicities, for any $\eta \in(0, \alpha)$ if $j \in J$ and for any $\eta \in\left(\omega \mathrm{e}^{s}, \alpha\right)$ if $j=0$.

Proof. Theorem 1.5.4(c) implies that $M \otimes F_{\eta}$ has pure $\partial_{j}$-radii $\omega \mathrm{e}^{s}$, for any $\eta \in(0, \alpha]$ if $j \in J$ and for any $\eta \in\left(\omega \mathrm{e}^{s}, \alpha\right]$ if $j=0$. The proposition then follows from the same argument as in Theorem 1.5.10, but invoking [Kedlaya and Xiao 2010, Theorem 2.3.10] in place of Theorem 1.5.4(e) when making the decomposition by 
extrinsic radii. Note also that we will only make use of the $\partial_{j}$-differential module $\mathscr{L}_{x, 0,(n)}^{(j)}$ in the proof which is defined over the entire disc $A_{K}^{1}[0, \alpha)$.

Proposition 1.5.17. Assume that $\left|u_{j}\right|=1$ for any $j \in J$. Let $M$ be a $\partial_{J^{+}}$-differential module over an open disc $A_{K}^{1}[0, \alpha)$ with $\alpha<1$. Assume that $M \otimes F_{\mathrm{e}^{-r}}$ has pure extrinsic radii $\min \left\{\omega \mathrm{e}^{s}, \mathrm{e}^{-r}\right\}$ for any $r>-\log \alpha$, where $\omega \mathrm{e}^{s}<\alpha$. Then there exists a unique direct sum decomposition $M=\bigoplus_{\{\hat{\vartheta}\}} M_{\{\hat{\vartheta}\}}$ of $\partial_{J^{+}-\text {differential modules }}$ over $A_{K}^{1}[0, \alpha)$, where the direct sum is taken over all $\mathrm{Gal}\left(K^{\mathrm{alg}} / K\right)$-orbits $\{\hat{\vartheta}\}$ in

$$
\bigoplus_{j \in J} \kappa_{K^{\text {alg }}}^{(s)} d u_{j} \oplus \kappa_{K^{\text {alg }}}^{(s)} d t
$$

such that the multiset of refined extrinsic radii of $M_{\{\hat{\vartheta}\}} \otimes F_{\eta}$ exactly consists of the $\operatorname{Gal}\left(K^{\mathrm{alg}} / K\right)$-orbits $\{\hat{\vartheta}\}$ with appropriate multiplicities, for any $\eta>\omega \mathrm{e}^{s}$.

Proof. Without loss of generality, we assume that $M$ is not a direct sum of two nonzero $\partial_{J^{+}}$-differential modules. We first show a dichotomy, similar to Theorem 1.5.6(f): for each $\partial_{j}$, either $M \otimes F_{\eta}$ has pure $\partial_{j}$-radii $\omega \mathrm{e}^{s}$ for all $\eta>\omega \mathrm{e}^{s}$, or $R_{\partial_{j}}\left(M \otimes F_{\eta}\right)<\omega \mathrm{e}^{s}$ for all $\eta>\omega \mathrm{e}^{s}$. Assume that we are not in the latter case. Then $R_{\partial_{j}}\left(M \otimes F_{\eta}\right)=E R\left(M \otimes F_{\eta}\right)$ for some $\eta \in\left(\omega \mathrm{e}^{s}, \alpha\right)$. By parts (c) and (d) of Theorem 1.5.4, the monotonicity and convexity of $f_{1}^{(j)}(M, r)$ forces $R_{\partial_{j}}\left(M \otimes F_{\eta}\right)=E R\left(M \otimes F_{\eta}\right)$ for all $\eta \in(0, \alpha)$. Now, if $R_{\partial_{j}}\left(M \otimes F_{\eta} ; 2\right)>E R\left(M \otimes F_{\eta}\right)$ for all $\eta \in\left(\omega \mathrm{e}^{s}, \alpha\right)$, we may use [Kedlaya and Xiao 2010, Theorem 2.3.10] to decompose $M$ to split off the smallest $\partial_{j}$-radii, which contradicts the indecomposability assumption on $M$. Therefore, $R_{\partial_{j}}\left(M \otimes F_{\eta} ; 2\right)=E R\left(M \otimes F_{\eta}\right)$ for some $\eta \in\left(\omega \mathrm{e}^{s}, \alpha\right)$. Continuing this argument for the third and other subsidiary $\partial_{j}$-radii leads us to the former case of the claim. The proposition now follows from applying Proposition 1.5.16 to each $\partial_{j}$ that satisfies the former condition of the claim.

Remark 1.5.18. We do not expect a decomposition theorem analogous to Proposition 1.5.17 in the case when the functions for extrinsic radii are linear with negative slopes. The reason is that, when $\eta$ is sufficiently close to $0, E R\left(M \otimes F_{\eta}\right)$ is always the same as $\eta$, and hence no information about the $\partial_{j}$-radii of $M \otimes F_{\eta}$ is reflected in the extrinsic radii. In contrast, in the situation of Proposition 1.5.17 if the functions of extrinsic radii stay constant before the they become equal to $-\log \eta$, all dominant $\partial_{j}$ must have constant $\partial_{j}$-radii by the monotonicity (Theorem 1.5.4(c)).

1.6. Refined differential conductors. Differential modules defined over an open annulus with outer radius 1 are historically considered very important, in particular those whose intrinsic radii approach 1, as we base change to the completion with respect to the Gauss norms with radii approaching to 1; this is known as the solvable case. In particular, the rate of the such change of intrinsic radii is related to the Swan conductors if the differential modules come from a Galois representation of $G_{\mathbb{F}_{p}((t))}$. In this subsection, we focus on this situation and define differential 
conductors, as well as refined differential conductors if the differential module has pure differential conductors.

We continue to assume Hypothesis 1.5.1. Moreover, we assume $p>0$ in this subsection.

Definition 1.6.1. Let $M$ be a $\partial_{J^{+}}$-differential module of rank $d$ over $A_{K}^{1}\left(\eta_{0}, 1\right)$ for some $\eta_{0} \in(0,1)$. We say that $M$ is solvable if $\operatorname{IR}\left(M \otimes F_{\eta}\right) \rightarrow 1$ as $\eta \rightarrow 1^{-}$.

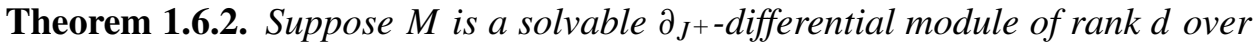
$A_{K}^{1}\left(\eta_{0}, 1\right)$, for some $\eta_{0} \in(0,1)$. Then by making $\eta_{0}$ sufficiently close to 1 , there exists a unique direct sum decomposition $M=M_{1} \oplus \cdots \oplus M_{\gamma}$ over $A_{K}^{1}\left(\eta_{0}, 1\right)$ and nonnegative distinct rational numbers $b_{1}, \ldots, b_{\gamma}$ with $b_{i} \cdot \operatorname{rank}\left(M_{i}\right) \in \mathbb{Z}$, such that $M_{i} \otimes F_{\eta}$ has pure intrinsic radii $\eta^{b_{i}}$ for any $i=1, \ldots, \gamma$ and any $\eta \in\left(\eta_{0}, 1\right)$.

Keep the same hypothesis and assume moreover that $\left|u_{j}\right|=1$ for all $j \in J$. Then by making $\eta_{0}$ sufficiently close to 1 , there exists a unique direct sum decomposition $M=\hat{M}_{1} \oplus \cdots \oplus \hat{M}_{\hat{\gamma}}$ over $A_{K}^{1}\left(\eta_{0}, 1\right)$ and nonnegative distinct rational numbers $\hat{b}_{1}, \ldots, \hat{b}_{\hat{\gamma}}$ with $\hat{b}_{i} \cdot \operatorname{rank}\left(\hat{M}_{i}\right) \in \mathbb{Z}$, such that $\hat{M}_{i} \otimes F_{\eta}$ has pure extrinsic radii $\eta^{\hat{b}_{i}}$ for any $i=1, \ldots, \hat{\gamma}$ and any $\eta \in\left(\eta_{0}, 1\right)$.

Proof. By parts (a), (b), and (d) of Theorem 1.5.6, for $l=1, \ldots, d$, the functions $d ! F_{l}(M, r)$ and $d ! \hat{F}_{l}(M, r)$ on $\left(0,-\log \eta_{0}\right)$ are continuous, convex, and piecewise affine with integer slopes. The assumption $d ! F_{l}(M, r) \rightarrow 0$ also implies that $d ! \hat{F}_{l}(M, r) \rightarrow 0$ as $r \rightarrow 0^{+}$; because of this and the fact that $d ! F_{l}(M, r) \geq 0$ and $d ! \hat{F}_{l}(M, r) \geq 0$ for all $r$, the slopes of $F_{l}(M, r)$ and $\hat{F}_{l}(M, r)$ are forced to be nonnegative. Hence there is a least such slope; that is, $d ! F_{l}(M, r)$ and $d ! \hat{F}_{l}(M, r)$ are linear in a right neighborhood of $r=0$.

We can thus choose $\eta_{0} \in(0,1)$ so that $d ! F_{l}(M, r)$ and $d ! \hat{F}_{l}(M, r)$ are linear on $\left(0,-\log \eta_{0}\right)$ for $l=1, \ldots, d$. The desired decomposition is constructed in Theorem 1.5.6(e) and the integrality of $b_{i} \cdot \operatorname{rank}\left(M_{i}\right)$ and $\hat{b}_{i} \cdot \operatorname{rank}\left(\hat{M}_{i}\right)$ follows from the fact that $F_{\operatorname{dim} M_{i}}\left(M_{i}, r\right)$ and $\hat{F}_{\operatorname{dim} \hat{M}_{i}}\left(\hat{M}_{i}, r\right)$ have integral slopes, again by Theorem 1.5.6(b).

Definition 1.6.3. Let $M$ be a solvable $\partial_{J^{+}}$-differential module of rank $d$ over $A_{K}^{1}\left(\eta_{0}, 1\right)$ for some $\eta_{0} \in(0,1)$. Define the multiset of differential log-breaks of $M$ to be the multiset consisting of $b_{i}$ from Theorem 1.6.2 with multiplicity rank $M_{i}$; we use $b_{\log }(M ; 1) \geq \cdots \geq b_{\log }(M ; d)$ to denote the differential log-breaks in decreasing order. We define the differential Swan conductor of $M$ to be the sum of the differential $\log$-breaks, that is, $\operatorname{Swan}(M)=\sum_{i=1}^{r} b_{i} \cdot \operatorname{rank}\left(M_{i}\right)$; it is a nonnegative integer by Theorem 1.6.2. We say that $M$ has pure differential log-breaks if all differential log-breaks are equal.

When $M$ has pure differential log-breaks, we define the multiset of refined Swan conductors of $M$, denoted by $\mathscr{I} \Theta(M)$, to be the multiset consisting of $\vartheta$ in (1.5.13) with multiplicity rank $M_{\vartheta}$. 
Similarly, when $\left|u_{j}\right|=1$ for all $j \in J$, we define the multiset of differential nonlogbreaks to be the multiset consisting of $\hat{b}_{i}$ from Theorem 1.6 .2 with multiplicity rank $\hat{M}_{i}$; we use $b_{\text {nlog }}(M ; 1) \geq \cdots \geq b_{\text {nlog }}(M ; d)$ to denote the differential nonlogbreaks in decreasing order. We define the differential Artin conductor of $M$ to be the sum of the differential nonlog-breaks; it is also a nonnegative integer by Theorem 1.6.2. We say that $M$ has pure differential nonlog-breaks if all differential nonlog-breaks are equal.

When $M$ has pure differential nonlog-breaks, we define the multiset of refined Artin conductors of $M$, denoted by $\mathscr{E} \Theta(M)$, to be the multiset of $\mu_{e} \rtimes \operatorname{Gal}\left(K^{\mathrm{sep}} / K\right)$ orbits $\left\{\mu_{e} \hat{\vartheta}\right\}$ in (1.5.15) with multiplicity equal to the multiplicities of $\left\{t^{-b} \mu_{e} \hat{\vartheta}\right\}$ in $M_{\left\{\mu_{e} \hat{\vartheta}\right\}} \otimes F_{\eta}$ for any $\eta \in\left(\eta_{0}, 1\right)$.

\section{Refined differential conductors for Galois representations}

One of the most important applications of $p$-adic differential modules is to provide an interpretation of the Swan conductors of representations of $G_{k}$, where $k$ is a complete discrete valuation field of equal characteristic $p>0$ with perfect residue field. This idea was later generalized by Kedlaya [2007] to the case when the residue field of $k$ need not to be perfect, and by the author [Xiao 2010] to relate the differential modules to the Swan conductors in the sense of Abbes and Saito [2002]. In this section, we further develop the theory on the differential module side to incorporate the study of refined differential conductors, which will be related to Saito's definition [2009] of refined Swan conductors, as proved in the next section.

Throughout this section, we assume that $p>0$ is a prime number.

2.1. Construction of differential modules. This subsection is dedicated to the construction of the differential modules associated to representations of $G_{k}$, where $k$ is a complete discrete valuation field of equal characteristic $p>0$.

Definition 2.1.1. For a field $\kappa$ of characteristic $p>0$, a $p$-basis of $\kappa$ is a set $\left(b_{j}\right)_{j \in J} \subset \kappa$ such that the products $b_{J}^{e_{J}}$, where $e_{j} \in\{0,1, \ldots, p-1\}$ for all $j \in J$ and $e_{j}=0$ for all but finitely many $j$, form a basis of the vector space $\kappa$ over $\kappa^{p}$.

Notation 2.1.2. Let $k$ be a complete discrete valuation field of characteristic $p>0$. Let $\pi_{k}$ be a uniformizer of $k$, generating the maximal ideal $\mathfrak{m}_{k}$ in the ring of integers $\mathscr{O}_{k}$. Let $\kappa=\kappa_{k}$ denote the residue field. Let $\bar{\kappa}=\kappa^{\text {alg }}$ denote an algebraic closure of $\kappa$. We choose and fix a noncanonical isomorphism $k \simeq \kappa\left(\left(\pi_{k}\right)\right)$. We fix a $p$-basis $\bar{b}_{J}$ of $\kappa$ and let $b_{J} \subset k$ be the preimage of them via the isomorphism above. Then $\left\{b_{J}, \pi_{k}\right\}$ form a $p$-basis of $k$, which we refer to as a lifted p-basis. Let $k_{0}=\bigcap_{n \in \mathbb{N}} \kappa^{p^{n}}=\bigcap_{n \in \mathbb{N}} k^{p^{n}}$. We know that $d \pi_{k}$ and $d b_{J}$ form a basis of $\Omega_{\mathscr{O}_{k}}^{1}$ over $\mathrm{O}_{k}$. 
Let $\mathscr{O}_{K}$ denote the Cohen ring of $\kappa$ with respect to $\bar{b}_{J}$ and let $B_{J} \subset \mathcal{O}_{K}$ be the canonical lifts of the $p$-basis. Put $K=$ Frac $O_{K}$. We use $\mathbb{O}_{K_{0}}$ to denote the ring of Witt vectors of $k_{0}$, viewed as a subring of $\mathcal{O}_{K}$ and we put $K_{0}=\mathfrak{O}_{K_{0}}\left[\frac{1}{p}\right]$.

Notation 2.1.3. For an extension $k^{\prime} / k$ of a complete discrete valuation field, the (naïve) ramification degree of $k^{\prime} / k$ is simply the index of the valuation of $k$ in that of $k^{\prime}$.

We say that $k^{\prime} / k$ is tamely ramified if $p \nmid e$ and the residue field extension $\kappa_{k^{\prime}} / \kappa_{k}$ is separable, that is, $\kappa_{k^{\prime}}$ is algebraic and separable over $\kappa_{k}\left(x_{\alpha} ; \alpha \in \Lambda\right)$ for some transcendental elements $x_{\alpha}$ and an index set $\Lambda$. If moreover, $e=1$, we say $k^{\prime} / k$ is unramified.

Notation 2.1.4. By a representation of $G_{k}$, we mean a continuous homomorphism $\rho: G_{k} \rightarrow \mathrm{GL}\left(V_{\rho}\right)$, where $V_{\rho}$ is a vector space over a (topological) field $F$ of characteristic zero. We say that $\rho$ is a $p$-adic if $F$ is a finite extension of $\mathbb{Q}_{p}$.

Let $F$ be a finite extension of $\mathbb{Q}_{p}$, let $\mathcal{O}$ denote its ring of integers, and let $\mathbb{F}_{q}$ denote the residue field of 0 , where $q$ is a power of $p$. Put $\mathbb{Z}_{q}=W\left(\mathbb{F}_{q}\right)$ and $\mathbb{Q}_{q}=\mathbb{Z}_{q}\left[\frac{1}{p}\right]$. By an 0-representation of $G_{k}$, we mean a continuous homomorphism $\rho: G_{k} \rightarrow \operatorname{GL}\left(\Lambda_{\rho}\right)$ with $\Lambda_{\rho}$ a finite free 0-module.

For $\rho$ a $p$-adic representation or an 0 -representation, we say that $\rho$ has finite local monodromy if the image of the inertia group $I_{k}$ is finite.

We assume that $\mathbb{F}_{q} \subseteq k_{0}$. Put $K^{\prime}=K F$. Since $F / \mathbb{Q}_{q}$ is totally ramified, we have $0_{K^{\prime}} \cong 0_{K} \otimes_{\mathbb{Z}_{q}} 0$.

Notation 2.1.5. We put $\mathscr{R}_{K^{\prime}}^{\eta}=K^{\prime}\langle\eta / T, T\}$ for $\eta \in(0,1)$ and $\mathscr{R}_{K^{\prime}}=\bigcup_{\eta \in(0,1)} \mathscr{R}_{K^{\prime}}^{\eta}$; the latter ring is commonly called the Robba ring over $K^{\prime}$. Let $\mathscr{R}_{K^{\prime}}^{\text {int }}$ be the subring of $\mathscr{R}_{K^{\prime}}$ consisting of elements whose 1 -Gauss norm is bounded by 1 ; it is a Henselian discrete valuation ring, with residue field $k$ if we identify the reduction of $T$ with $\pi_{k}$. For $\eta \in(0,1)$, we use $F_{\eta}^{\prime}$ to denote the completion of $K^{\prime}(T)$ with respect to the $\eta$-Gauss norm.

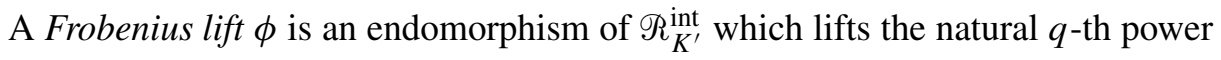
Frobenius on $k$. Any Frobenius lift extends by continuity to an action on $\mathscr{R}_{K^{\prime}}$. A standard Frobenius lift is a Frobenius lift which sends $T$ to $T^{p}$ and $B_{j}$ to $B_{j}^{p}$ for any $j \in J$.

The differentials

$$
\Omega_{\mathscr{R}_{K^{\prime}}^{\text {int }}}^{1}, \quad \Omega_{\mathscr{R}_{K^{\prime}}}^{1} \quad \text { and } \quad \Omega_{\mathscr{R}_{K^{\prime}}^{\eta}}^{1}
$$

for any $\eta \in(0,1)$ admit a basis given by $d B_{J}$ and $d T$. We set $\partial_{0}=\partial / \partial T, \partial_{j}=\partial / \partial B_{j}$ with $j \in J$ for the dual basis. Then a $\nabla$-module over $\mathscr{R}_{K^{\prime}}$ is just a $\partial_{J^{+}}$-differential module. 
Definition 2.1.6. Let $\phi$ be a Frobenius lift. For $R=\mathscr{R}_{K^{\prime}}, \mathscr{R}_{K^{\prime}}^{\eta}$, or $\mathscr{R}_{K^{\prime}}^{\text {int a }}(\phi, \nabla)$ module $M$ over $R$ is a $\partial_{J^{+-}}$differential module together with an isomorphism $\Phi: \phi^{*} M \rightarrow M$ of $\partial_{J^{+}-\text {differential modules. }}$

Theorem 2.1.7. For any Frobenius lift $\phi$, we have an equivalence of categories between the category of 0 -representations with finite local monodromy and the category of $(\phi, \nabla)$-modules over $\Re_{K^{\prime}}^{\text {int }}$. Moreover, all $(\phi, \nabla)$-modules can be realized over $\Re_{K^{\prime}}^{\eta}$ for some $\eta \in(0,1)$. This $(\phi, \nabla)$-module is independent of the choice of the p-basis.

Proof. The functor is constructed in [Kedlaya 2007, Section 3; Xiao 2010, Section 2.2].

Definition 2.1.8. For a $p$-adic representation $\rho$ of $G_{k}$ with finite local monodromy, we choose an 0 -lattice $\Lambda_{\rho}$ of $V_{\rho}$, stable under the action of $G_{k}$; this gives an O-representation of $G_{k}$. Theorem 2.1.7 then produces a $(\phi, \nabla)$-module over $\mathscr{R}_{K^{\prime}}^{\text {int }}$, whose base change to $\mathscr{R}_{K^{\prime}}$ is called the differential module associated to $\rho$, denoted by $\mathscr{E}_{\rho}$. This $\mathscr{E}_{\rho}$ does not depend on the choice of the lattice $\Lambda_{\rho}$.

For the rest of this subsection, we assume the following.

Hypothesis 2.1.9. The residue field $\kappa$ has a finite $p$-basis $\bar{b}_{J}$, where $J=\{1, \ldots, m\}$. We put $J^{+}=J \cup\{0\}$.

Proposition 2.1.10. Let $\phi$ be the standard Frobenius lift on $\mathscr{R}_{K^{\prime}}^{\mathrm{int}}$. Then the Frobenius $\phi: F_{\eta^{q}}^{\prime} \rightarrow F_{\eta}^{\prime}$ is the same as the iterative Frobenius $\varphi^{\left(\partial_{0}, \lambda\right)} \circ \cdots \circ \varphi^{\left(\partial_{m}, \lambda\right)}$ in Construction 1.2.14, where $q=p^{\lambda}$.

Proof. We may assume that $K^{\prime}$ contains $\zeta_{q}$, a $q$-th root of unity. It suffices to show that the image $\phi\left(F_{\eta^{q}}^{\prime}\right)$ is stable under the action of $(\mathbb{Z} / q \mathbb{Z})^{m+1}$ in the sense of Construction 1.2.14, where each $\partial_{j}$-Frobenius corresponds to a factor $\mathbb{Z} / q \mathbb{Z}$, and that the degree of $F_{\eta}^{\prime}$ over $\phi\left(F_{\eta^{q}}^{\prime}\right)$ is $q^{m+1}$.

For $\underline{i}=\left(i_{0}, \ldots, i_{m}\right) \in(\mathbb{Z} / q \mathbb{Z})^{m+1}$, we have $T^{(i)}=\zeta_{q}^{i_{0}} T$ and $\left(B_{j}\right)^{(i)}=\zeta_{q}^{i_{j}} B_{j}$ for any $j \in J$. Hence $(\cdot)^{(\underline{i})} \circ \phi$ for all $\underline{i}$ are continuous homomorphisms from $\mathcal{O}_{K} \llbracket T \rrbracket$ to itself, sending $B_{j}$ to $B_{j}^{q}$ and $T$ to $T^{q}$. By the functoriality of Cohen rings (see [Xiao 2010, Proposition 2.1.8]), these homomorphisms are all the same. Hence the image of $\phi$ is stable under the $(\mathbb{Z} / q \mathbb{Z})^{m+1}$-action. It is evident that $F_{\eta}^{\prime}$ has rank $q^{m+1}$ over $\phi\left(F_{\eta^{q}}^{\prime}\right)$; this forces the two homomorphisms to be the same.

Proposition 2.1.11. Let $\phi$ be the standard Frobenius lift on $\mathscr{R}_{K^{\prime}}^{\text {int }}$ and let $\mathscr{E}$ be a $(\phi, \nabla)$-module over $A_{K^{\prime}}^{1}\left[\eta_{0}, 1\right)$ for some $\eta_{0} \in(0,1)$. Then $\mathscr{E}$ is solvable.

Proof. This is well-known to the experts; we include a proof for the convenience of the reader. By Lemma 1.2.18(a), we have

$f_{i}\left(\phi^{*} M, r\right)$

$$
=\max \left\{p^{-\lambda} f_{i}(M, q r), p^{1-\lambda}\left(f_{i}(M, q r)-\log p\right), \ldots, f_{i}(M, q r)-\lambda \log p\right\},
$$


where $\lambda=\log _{p} q$. Since $\phi^{*} M \stackrel{\sim}{\longrightarrow} M$, the function $g_{i}(M)=\lim \sup _{r \rightarrow 0^{+}} f_{i}(M, r)$ satisfies

$$
g_{i}(M)=\max \left\{p^{-\lambda} g_{i}(M), p^{1-\lambda}\left(g_{i}(M)-\log p\right), \ldots, g_{i}(M)-\lambda \log p\right\} .
$$

This forces $g_{i}(M)$ to be zero. By the continuity of $f_{i}(M, r)$ and the convexity of $F_{i}(M, r)$ in Theorem 1.5.6, $\lim _{r \rightarrow 0^{+}} f_{i}(M, r)=0$. In other words, $\mathscr{E}$ is solvable.

Proposition 2.1.12. Let $\phi$ be the standard Frobenius lift and let $\phi^{\prime}$ be another Frobenius lift on $\mathscr{R}_{K^{\prime}}^{\mathrm{int}}$. Assume that $\mathscr{E}$ is a $(\phi, \nabla)$-module over $A_{K^{\prime}}^{1}\left[\eta_{0}, 1\right)$ for some $\eta_{0} \in(0,1)$. Then the restriction of $\mathscr{E}$ to $A_{K^{\prime}}^{1}[\eta, 1)$ for some $\eta \in\left[\eta_{0}, 1\right)$ is naturally equipped with a $\left(\phi^{\prime}, \nabla\right)$-module structure.

Proof. Define the Frobenius structure for $\phi^{\prime}$ by Taylor series as follows. For $\boldsymbol{v} \in \mathscr{E}$, $\phi^{\prime}(v)$

$$
=\sum_{e_{J^{+}}=0}^{\infty} \frac{\left(\phi^{\prime}(T)-\phi(T)\right)^{e_{0}} \prod_{j \in J}\left(\phi^{\prime}\left(B_{j}\right)-\phi\left(B_{j}\right)\right)^{e_{j}}}{\left(e_{J^{+}}\right) !} \phi\left(\frac{\partial^{e_{0}}}{\partial T^{e_{0}}} \frac{\partial^{e_{1}}}{\partial B_{1}^{e_{1}}} \cdots \frac{\partial^{e_{m}}}{\partial B_{m}^{e_{m}}}(\boldsymbol{v})\right) .
$$

Since $\left|\phi^{\prime}(T)-\phi(T)\right|_{1}<1$ and $\left|\phi^{\prime}\left(B_{j}\right)-\phi\left(B_{j}\right)\right|_{1}<1$ for all $j \in J$, we have the same inequality using $\eta$-Gauss norm when $\eta \in\left[\eta_{0}^{\prime}, 1\right]$ for some $\eta_{0}^{\prime}$ sufficiently close to 1 . Hence the expression for $\phi^{\prime}$ converges on $A_{K^{\prime}}^{1}\left[\eta_{0}^{\prime}, 1\right)$ and gives the restriction of $\mathscr{E}$ to $A_{K^{\prime}}^{1}\left[\eta_{0}^{\prime}, 1\right)$ a structure of $\left(\phi^{\prime}, \nabla\right)$-module.

Remark 2.1.13. One may also approach the results of this subsection without referring to the standard Frobenius but instead using a generalized version of Lemma 1.2.18(a) for noncentered Frobenius. This point of view is taken in [Kedlaya 2010, Chapter 17].

2.2. Differential conductors. Combining the results from Section 1.6 and Proposition 2.1.11, we can define differential conductors for a representation of $G_{k}$ with finite local monodromy. To make this definition more robust, we will introduce the break with respect to each element of the $p$-basis, and the break of the differential module is just the maximum among all breaks for each element of the $p$-basis, after appropriate normalization. This point of view is in particular useful when we try to understand how the conductors change when restricting a Galois representation to $G_{l}$ for some (explicit) finite extension $l$ of $k$.

Definition 2.2.1. We first assume that $k$ satisfies Hypothesis 2.1.9. Let $\rho$ be a representation of $G_{k}$ with finite local monodromy. The log-breaks of $\rho$ are defined to be the differential log-breaks of $\mathscr{E}_{\rho}$, as a solvable $\partial_{J^{+}}$-differential module. Put

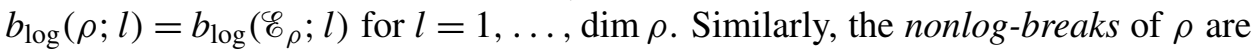
defined to be the differential nonlog-breaks of $\mathscr{E}_{\rho} \rho \rho^{I_{k}}$ together with the element 0 with multiplicity $\operatorname{dim} \rho^{I_{k}}$, where $\rho^{I_{k}}$ is the maximal subrepresentation of $\rho$ on which $I_{k}$ acts trivially. Put $b_{\text {nlog }}(\rho ; l)=b_{\operatorname{nlog}}\left(\mathscr{E}_{\rho / \rho^{I_{k}}} ; l\right)$ for $l=1, \ldots, \operatorname{dim}\left(\rho / \rho^{I_{k}}\right)$, 
and $b_{\mathrm{n} \log }\left(\rho ; \operatorname{dim}\left(\rho / \rho^{I_{k}}\right)+1\right)=\cdots=b_{\mathrm{nlog}}(\rho ; \operatorname{dim} \rho)=0$.

For simplicity, we also put $b_{\text {nlog }}(\rho)=b_{\text {nlog }}(\rho ; 1)$ and $b_{\log }(\rho)=b_{\log }(\rho ; 1)$; they are called the highest nonlog-break and the highest log-break, respectively.

We now consider a general $k$. For a $p$-adic representation $\rho$ of $G_{k}$ with finite local monodromy, let $l$ be the extension of $k$ corresponding to $\operatorname{Ker} \rho$ via Galois theory. We may choose a $p$-basis $\left\{c_{J}, \pi_{l}\right\}$ of $l$ such that $\pi_{l}$ is a uniformizer and $c_{J} \subset \mathbb{O}_{l}^{\times}$, and such that $c_{J \backslash J_{0}} \subset \mathcal{O}_{k}$ for some finite subset $J_{0} \subset J$. If we use $k^{\wedge}$ to denote the completion of $k\left(c_{J \backslash J_{0}}^{1 / p^{n}} ; n \in \mathbb{N}\right)$, then $k^{\wedge}$ verifies Hypothesis 2.1.9. We define the nonlog-breaks and log-breaks of $\rho$ to be, respectively, those of $\left.\rho\right|_{G_{k^{\wedge}}}$. Their sums are called the Artin conductors and, respectively, Swan conductors of $\rho$, denoted by $\operatorname{Art}(\rho)$ and $\operatorname{Swan}(\rho)$. These do not depend on the choice of the $p$-basis or of $J_{0}$, by [Kedlaya 2007, Proposition 2.6.6].

Definition 2.2.2. Put Fil ${ }^{0} G_{k}=G_{k}$ and $\mathrm{Fil}^{a} G_{k}=I_{k}$ for $a \in(0,1]$. For $a>1$, let $R_{a}$ be the set of finite image representations $\rho$ with nonlog-break strictly less than $a$. Put Fil ${ }^{a} G_{k}=\bigcap_{\rho \in R_{a}}\left(I_{k} \cap \operatorname{ker}(\rho)\right)$ and set Fil ${ }^{a+} G_{k}$ to be the closure of $\bigcup_{b>a}$ Fil $^{b} G_{k}$. This defines a filtration on $G_{k}$ such that for any representation $\rho$ with finite image, $\rho\left(\right.$ Fil $\left.^{a} G_{k}\right)$ is trivial if and only if $\rho \in R_{a}$.

Similarly, put $\mathrm{Fil}_{\log }^{0} G_{k}=G_{k}$. For $a>0$, let $R_{a, \log }$ be the set of finite image representations $\rho$ with log-break less than $a$. Put Fil $\log _{\log }^{a} G_{k}=\bigcap_{\rho \in R_{a, \mathrm{log}}}\left(I_{k} \cap \operatorname{ker}(\rho)\right)$ and set $\mathrm{Fil}_{\log }^{a+} G_{k}$ to be the closure of $\bigcup_{b>a} \mathrm{Fil}_{\log }^{b} G_{k}$. This defines a filtration on $G_{k}$ such that for any representation $\rho$ with finite image, $\rho\left(\mathrm{Fil}_{\log }^{a} G_{k}\right)$ is trivial if and only if $\rho \in R_{a, \log }$.

For a finite Galois extension $l$ of $k$, the above filtrations induce filtrations on the Galois group $G_{l / k}$ by

$$
G_{l / k,(\log )}^{a}=G_{l} \mathrm{Fil}_{(\log )}^{a} G_{k} / G_{l} \quad \text { and } \quad G_{l / k,(\log )}^{a+}=G_{l} \mathrm{Fil}_{(\log )}^{a+} G_{k} / G_{l},
$$

for $a \geq 0$. We define the (log-)ramification breaks of the extension $l / k$ to be the numbers $b$ for which $G_{l / k(, \log )}^{b} \neq G_{l / k(, \log )}^{b+}$. We order them as

$$
b_{(\mathrm{n}) \log }(l / k)=b_{(\mathrm{n}) \log }(l / k ; 1) \geq b_{(\mathrm{n}) \log }(l / k ; 2) \geq \cdots .
$$

In particular, if $\rho$ is a faithful representation of $G_{l / k}$, we have $b_{(\mathrm{n}) \log }(\rho)=b_{(\mathrm{n}) \log }(l / k)$.

Theorem 2.2.3. The differential conductors satisfy the following properties:

(a) For any representation $\rho$ of finite local monodromy,

$$
\begin{gathered}
\operatorname{Art}(\rho)=\sum_{a \in \mathbb{Q}_{\geq 0}} a \cdot \operatorname{dim}\left(V_{\rho}^{\mathrm{Fil}^{a+} G_{k}} / V_{\rho}^{\mathrm{Fil}^{a} G_{k}}\right) \in \mathbb{Z}_{\geq 0}, \\
\operatorname{Swan}(\rho)=\sum_{a \in \mathbb{Q}_{\geq 0}} a \cdot \operatorname{dim}\left(V_{\rho}^{\mathrm{Fil}_{\log }^{a+} G_{k}} / V_{\rho}^{\mathrm{Fil}_{\log }^{a} G_{k}}\right) \in \mathbb{Z}_{\geq 0} .
\end{gathered}
$$

(b) Let $k^{\prime} / k$ be a (not necessarily finite) extension of complete discretely valued 
fields. If $k^{\prime} / k$ is unramified, then $\mathrm{Fil}^{a} G_{k^{\prime}}=\mathrm{Fil}^{a} G_{k}$ for $a>0$. If $k^{\prime} / k$ is tamely ramified with naïve ramification index $e<\infty$, then $\mathrm{Fil}_{\log }^{e a} G_{k^{\prime}}=\mathrm{Fil}_{\log }^{a} G_{k}$ for $a>0$.

(c) For $a>0$, we have $\mathrm{Fil}^{a+1} G_{k} \subseteq \mathrm{Fil}_{\log }^{a} G_{k} \subseteq \mathrm{Fil}^{a} G_{k}$.

(d) For graded pieces we have, for $a>1$,

$$
\text { Fil }^{a} G_{k} / \mathrm{Fil}^{a+} G_{k}= \begin{cases}0 & \text { if } a \notin \mathbb{Q}, \\ \text { an abelian group killed by } p & \text { if } a \in \mathbb{Q},\end{cases}
$$

and for $a>0$,

$$
\mathrm{Fil}_{\log }^{a} G_{k} / \mathrm{Fil}_{\log }^{a+} G_{k}= \begin{cases}0 & \text { if } a \notin \mathbb{Q}, \\ \text { an abelian group killed by } p & \text { if } a \in \mathbb{Q} .\end{cases}
$$

(e) These filtrations on $G_{k}$ agree with the ones defined in [Abbes and Saito 2002, 2003].

Proof. Using the comparison [Xiao 2010, Theorem 4.4.1] of the arithmetic and differential conductors, this follows from their basic properties as stated in [Xiao 2010, Theorem 2.4.1 and Proposition 4.1.7]. We refer to [Xiao 2010; Abbes and Saito 2002] for the definition of Abbes and Saito's filtrations.

We now study the break for each element of the $p$-basis. We assume the validity of Hypothesis 2.1.9 for the rest of the subsection.

Proposition 2.2.4. For each $j \in J^{+}$, there is a ramification break $b_{j}(\rho)$ associated to $b_{j}(j \in J)$ or $\pi_{k}(j=0)$, such that $R_{\partial_{j}}\left(\mathscr{E}_{\rho} \otimes F_{\eta}^{\prime}\right)=\eta^{b_{j}(\rho)}$ for any $\eta \in\left(\eta_{0}, 1\right)$ with some $\eta_{0}<1$. Moreover,

$$
b_{\text {nlog }}(\rho)=\max _{j \in J^{+}}\left\{b_{j}(\rho)\right\}, \quad b_{\log }(\rho)=\max \left\{b_{0}(\rho)-1 ; b_{j}(\rho) \text { for } j \in J\right\} .
$$

Proof. By applying the same argument of Proposition 2.1.11 to intrinsic $\partial_{j}$-radii, we know that

$$
I R_{\partial_{j}}\left(\mathscr{E}_{\rho} \otimes F_{\eta^{q}}^{\prime}\right)=I R_{\partial_{j}}\left(\mathscr{E}_{\rho} \otimes F_{\eta}^{\prime}\right)^{q}
$$

as $\eta \rightarrow 1^{-}$. Therefore, by the convexity given by Theorem $1.5 .4(\mathrm{~d}), f_{1}^{(j)}\left(\mathscr{E}_{\rho}, r\right)$ is affine as $r \rightarrow 0^{+}$. The proposition follows.

Definition 2.2.5. We call $b_{J^{+}}(\rho)$ the breaks by p-basis of $\rho$ with respect to the lifted $p$-basis $b_{J}$ and the uniformizer $\pi_{k}$.

Remark 2.2.6. Rigorously speaking, the breaks by $p$-basis depend on the choice of the dual basis $\partial_{0}, \ldots, \partial_{m}$ of the differential forms. So when we change the choices of the lifted $p$-basis and the uniformizer, the breaks by basis $b_{j}(\rho)$ may change accordingly. 
Lemma 2.2.7. Fix $j_{0} \in J$. Let $b_{J^{+}}^{\prime}(\rho)$ be the breaks by p-basis of $\rho$ with respect to the lifted p-basis $\left\{b_{J \backslash\left\{j_{0}\right\}}, b_{j_{0}}+\pi_{k}\right\}$ and the uniformizer $\pi_{k}$. Then $b_{j}^{\prime}(\rho)=b_{j}(\rho)$ for $j \in J$ and

$$
b_{0}^{\prime}(\rho) \begin{cases}=\max \left\{b_{0}(\rho), b_{j_{0}}(\rho)\right\} & \text { if } b_{0}(\rho) \neq b_{j_{0}}(\rho), \\ \leq b_{0}(\rho) & \text { if } b_{0}(\rho)=b_{j_{0}}(\rho) .\end{cases}
$$

Proof. Let $\partial_{J^{+}}^{\prime}$ denote the derivations dual to the basis $d B_{J \backslash\left\{j_{0}\right\}}, d T, d\left(B_{j_{0}}+T\right)$ of $\Omega_{\mathscr{R}_{K^{\prime}}^{\text {int }}}^{1}$. Then $\partial_{J}^{\prime}=\partial_{J}$ and $\partial_{0}^{\prime}=\partial_{0}-\partial_{j_{0}}$. The lemma follows immediately.

Remark 2.2.8. This lemma is in fact much stronger than it appears. Applying the same argument to $b_{j_{0}}+\alpha \pi_{k}$ for all $\alpha \in k_{0}$ implies that, for all but possibly one $\alpha \in k_{0}, b_{0}^{\prime}(\rho) \geq b_{j_{0}}(\rho)$. So, vaguely speaking, the equality $b_{0}(\rho)=b(\rho)$ holds "generically"; this motivates the following lemma.

Lemma 2.2.9. Fix $j_{0} \in J$. Let $\tilde{k}$ be the completion of $k(x)$ with respect to the 1-Gauss norm, equipped with the lifted p-basis $\left\{b_{J \backslash\left\{j_{0}\right\}}, b_{j_{0}}+x \pi_{k}, x\right\}$. Let $\tilde{\rho}$ be the representation $G_{\tilde{k}} \rightarrow G_{k} \stackrel{\rho}{\rightarrow} G L\left(V_{\rho}\right)$. Let $\tilde{b}_{J+\cup\{m+1\}}(\tilde{\rho})$ denote the breaks by p-basis with respect to the aforementioned lifted p-basis and the uniformizer $\pi$, where $\tilde{b}_{J \backslash\left\{j_{0}\right\}}(\tilde{\rho})$ corresponds to $b_{J \backslash\left\{j_{0}\right\}}, \tilde{b}_{j_{0}}(\tilde{\rho})$ corresponds to $b_{j_{0}}+x \pi_{k}, \tilde{b}_{0}(\tilde{\rho})$ corresponds to $\pi_{k}$, and $\tilde{b}_{m+1}(\tilde{\rho})$ corresponds to $x$. Then $\tilde{b}_{j}\left(\rho^{\prime}\right)=b_{j}(\rho)$ for $j \in J$, $\tilde{b}_{m+1}(\tilde{\rho})=b_{j_{0}}(\rho)-1$, and $\tilde{b}_{0}(\tilde{\rho})=\max \left\{b_{0}(\rho), b_{j_{0}}(\rho)\right\}$. In particular, $\tilde{b}_{\mathrm{nlog}}(\tilde{\rho})=$ $b_{\text {nlog }}(\rho)$.

Proof. Let $\widetilde{K}^{\prime}$ denote the completion of $K^{\prime}(X)$ with respect to the 1-Gauss norm, where $X$ is the canonical lift of $x$. Let $f: A_{\widetilde{K}^{\prime}}^{1}\left[\eta_{0}, 1\right) \rightarrow A_{K^{\prime}}^{1}\left[\eta_{0}, 1\right)$ be the natural morphism. Then $f^{* \mathscr{E}_{\rho}}$ is the differential module associated to $\rho^{\prime}$. Let $\tilde{\partial}_{J^{+} \cup\{m+1\}}$ be the differential operators corresponding to the $p$-basis $\left(b_{J \backslash\left\{j_{0}\right\}}, b_{j_{0}}+x \pi_{k}, \pi_{k}\right)$. Then under the identification by $f^{*}$, we have

$$
\tilde{\partial}_{J}=\partial_{J}, \quad \tilde{\partial}_{m+1}=T \partial_{j_{0}}, \quad \tilde{\partial}_{0}=\partial_{0}-X \partial_{j_{0}} .
$$

The lemma follows from this because $X$ is transcendental over $K^{\prime}$.

Lemma 2.2.11. Fix $j_{0} \in J$. Set $k^{\prime}=k\left(b_{j_{0}}^{1 / p}\right)$, equipped with the lifted $p$-basis $\left\{b_{J \backslash\left\{j_{0}\right\}}, b_{j_{0}}^{1 / p}\right\}$. Let $b_{J^{+}}^{\prime}\left(\left.\rho\right|_{G_{k^{\prime}}}\right)$ be the breaks by p-basis of $\left.\rho\right|_{G_{k^{\prime}}}$ with respect to the aforementioned p-basis and uniformizer $\pi_{k}$. Then $b_{j}^{\prime}\left(\left.\rho\right|_{G_{k^{\prime}}}\right)=b_{j}(\rho)$ for $j \in$ $J^{+} \backslash\left\{j_{0}\right\}$ and $b_{j_{0}}^{\prime}\left(\left.\rho\right|_{k_{k^{\prime}}}\right)=\frac{1}{p} b_{j_{0}}(\rho)$.

Proof. Replacing $k$ by $k^{\prime}$ is equivalent to pulling back the differential module $\mathscr{E}_{\rho}$ along $\varphi^{\left(\partial_{j}\right)}$. The lemma follows from applying Lemma 1.2.18(a) to $\mathscr{E} \otimes F_{\eta}^{\prime}$ when $\eta \rightarrow 1^{-}$.

Lemma 2.2.12. Fix $j_{0} \in J$. Let $k^{\prime}$ denote the completion of $k\left(b_{j_{0}}^{1 / p^{n}} ; n \in \mathbb{N}\right)$ equipped with lifted p-basis $b_{J \backslash\left\{j_{0}\right\}}$. Let $b_{J^{+}}^{\prime}\left(\left.\rho\right|_{G_{k^{\prime}}}\right)$ be the breaks by p-basis of $\left.\rho\right|_{G_{k^{\prime}}}$ with 
respect to this p-basis and the uniformizer $\pi_{k}$. Then we have $b_{j}^{\prime}\left(\left.\rho\right|_{G_{k^{\prime}}}\right)=b_{j}(\rho)$ for $j \in J^{+} \backslash\left\{j_{0}\right\}$.

Proof. Replacing $k$ with $k^{\prime}$ is equivalent to simply forgetting the $j_{0}$-direction.

Situation 2.2.13. Now, we study a particular case of base change, which will be useful in the comparison Theorem 3.4.1. This type of base change was first considered by Saito [2009].

Fix $e \in \mathbb{N}$ possibly divisible by $p$. Let $k$ be as above, and let $k^{\prime}$ be the completion of $k(x)$ with respect to the 1-Gauss norm, with uniformizer $\pi_{k^{\prime}}=\pi_{k}$. Put $\tilde{k}=k^{\prime}[u] /\left(u^{e}-x^{-1} \pi_{k}\right)$. The residue field of $\tilde{k}$ is $\kappa(\bar{x})$; we consider the $p$-basis $\left(b_{J}, \bar{x}\right)$ and the uniformizer $\pi_{\tilde{k}}=u$ of $\tilde{k}$. We choose the unique isomorphism $\kappa(\bar{x})((u)) \simeq \tilde{k}$ that is compatible with the chosen isomorphism $\kappa\left(\pi_{k}\right) \simeq k$ in Notation 2.1.2 and that sends $\bar{x}$ to $x$. This gives rise to the lifted $p$-basis $\left(b_{J}, x, u\right)$ of $\tilde{k}$.

Proposition 2.2.14. The natural homomorphism $G_{\tilde{k}} \rightarrow G_{k}$ induces a homomorphism $\mathrm{Fil}_{\log }^{e a} G_{\tilde{k}} \rightarrow \mathrm{Fil}_{\log }^{a} G_{k}$ for any $a \in \mathbb{Q}_{\geq 0}$. Moreover, the induced homomorphism

$$
\mathrm{Fil}_{\log }^{e a} G_{\tilde{k}} / \mathrm{Fil}_{\log }^{e a+} G_{\tilde{k}} \rightarrow \mathrm{Fil}_{\log }^{a} G_{k} / \mathrm{Fil}_{\log }^{a+} G_{k}
$$

is surjective for any $a \in \mathbb{Q}_{>0}$.

Proof. It suffices to show that, for a $p$-adic representation of $G_{k}$ with finite local monodromy and pure log-break $b_{\log }(\rho)$, the induced representation

$$
\tilde{\rho}: G_{\tilde{k}} \rightarrow G_{k} \rightarrow G L\left(V_{\rho}\right)
$$

also has the same log-break. Let $\widetilde{K}^{\prime}$ be the completion of $K^{\prime}(X)$ with respect to the 1-Gauss norm, where $X$ is the canonical lift of $\bar{x}$. We then have a natural map $f: A_{\widetilde{K}^{\prime}}^{1}\left[\eta^{1 / e}, 1\right) \rightarrow A_{K^{\prime}}^{1}[\eta, 1)$ for $\eta \rightarrow 1^{-}$, sending $T$ to $X U^{e}$, where $U$ is the coordinate of the former annulus.

Let $\tilde{b}_{0}(\tilde{\rho}), \ldots, \tilde{b}_{m+1}(\tilde{\rho})$ be the breaks by $p$-basis with respect to $b_{J}, x$ and the uniformizer $\pi_{\tilde{k}}=u$. Then $f^{* \mathscr{E}_{\rho}}$ is the differential module associated to $\tilde{\rho}$, with the actions of $\tilde{\partial}_{0}=\partial / \partial U, \tilde{\partial}_{J}=\partial / \partial B_{J}$, and $\tilde{\partial}_{m+1}=\partial / \partial X$. We have

$$
\tilde{\partial}_{J}=\partial_{J}, \quad \tilde{\partial}_{0}=e X U^{e-1} \partial_{0}, \quad \text { and } \tilde{\partial}_{m+1}=U^{e} \partial_{0} .
$$

By Theorem 1.4.20, we have $\tilde{b}_{J}(\tilde{\rho})=e b_{J}(\rho), \tilde{b}_{0}(\tilde{\rho}) \leq e b_{0}(\rho)-(e-1)$, and $\tilde{b}_{m+1}(\tilde{\rho})=e b_{0}(\rho)-e$ (when $e$ is prime to $p$, the inequality becomes an equality). In particular, we have $\tilde{b}_{m+1}(\tilde{\rho}) \geq \tilde{b}_{0}(\tilde{\rho})-1$. Hence we conclude that $b_{\log }(\tilde{\rho})=\max \left\{\tilde{b}_{0}(\tilde{\rho})-1, \tilde{b}_{J}(\tilde{\rho}), \tilde{b}_{m+1}(\tilde{\rho})\right\}=\max \left\{e b_{J}(\rho), e b_{0}(\rho)-e\right\}=e b_{\log }(\rho)$.

This proves the proposition. 
2.3. Refined differential conductors. In this subsection, we define the refined differential conductors, which provides additional information about the subquotient $\mathrm{Fil}_{(\log )}^{a} G_{k} / \mathrm{Fil}_{(\log )}^{a+} G_{k}$ of the ramification filtrations.

We keep the notation as in previous subsections but we drop Hypothesis 2.1.9.

Notation 2.3.1. Fix a Dwork pi $\pi=(-p)^{1 /(p-1)}$.

Notation 2.3.2. We put $\Omega_{\mathscr{O}_{k}}^{1}(\log )=\Omega_{\mathscr{O}_{k}}^{1}+\mathscr{O}_{k} \frac{d \pi_{k}}{\pi_{k}} \subset \Omega_{k}^{1}$. If we choose a $p$-basis $\bar{b}_{J}$ of $\kappa$ as in Notation 2.1.2, we have

$$
\Omega_{\mathscr{O}_{k}}^{1}(\log )=\mathscr{O}_{k} \frac{d \pi_{k}}{\pi_{k}} \oplus\left(\bigoplus_{j \in J} \mathscr{O}_{k} d b_{j}\right) .
$$

Construction 2.3.3. Let $\rho$ be a $p$-adic representation of $G_{k}$ with finite local monodromy and with pure break $b=b_{\operatorname{nlog}}(\rho)$ or $\log$-break $b=b_{\log }(\rho)$. We may replace $k$ by the completion of an inseparable extension as in Definition 2.2.1 and then assume Hypothesis 2.1.9. Let $\mathscr{E}_{\rho}$ denote the $(\phi, \nabla)$-module associated to $\rho$. By Theorem 1.5.6(e), there exists $\eta_{0} \in(0,1)$ such that $\mathscr{E}_{\rho} \otimes F_{\eta}^{\prime}$ has pure extrinsic or intrinsic, respectively, radii $\eta^{b}$ for any $\eta \in\left[\eta_{0}, 1\right)$.

We define the multiset of refined Artin conductors of $\rho$ to be

$$
\operatorname{rar}(\rho)=\left\{\frac{1}{\pi} \vartheta \pi_{k}^{-b}: \vartheta \in \mathscr{I} \Theta\left(\mathscr{E}_{\rho}\right)\right\} \subset \Omega_{\mathscr{O}_{k}}^{1} \otimes_{\mathscr{O}_{k}} \pi_{k}^{-b} \bar{\kappa} .
$$

Similarly, we define the multiset of refined Swan conductors of $\rho$ to be

$$
\operatorname{rsw}(\rho)=\left\{\frac{1}{\pi} \vartheta \pi_{k}^{-b}: \vartheta \in \mathscr{I} \Theta\left(\mathscr{E}_{\rho}\right)\right\} \subset \Omega_{\mathscr{O}_{k}}^{1}(\log ) \otimes_{\mathscr{O}_{k}} \pi_{k}^{-b} \bar{\kappa} .
$$

Remark 2.3.4. There is a unique primitive $p$-th root of unity $\zeta_{p}$ such that

$$
\pi \equiv\left(\zeta_{p}-1\right) \bmod \left(\zeta_{p}-1\right)^{2} .
$$

The definition of refined conductors above is unchanged if we replace $\pi$ by $\zeta_{p}-1$.

Lemma 2.3.5. In Construction 2.3.3, the definition of the refined Artin and Swan conductors does not depend on the choices of the lifted p-basis of $k$ and the uniformizer $\pi_{k}$.

Proof. We may assume Hypothesis 2.1.9 since only finitely many elements in the $p$-basis appear in the refined Artin and Swan conductors.

For another choice of lifted $p$-bases and uniformizers, we will consider another set of differential operators: $\partial_{j}^{\prime}=\partial / \partial B_{j}^{\prime}$ for $j \in J$ and $\partial_{0}^{\prime}=\partial / \partial T^{\prime}$. We put $d B_{j}=\sum_{j^{\prime} \in J} \alpha_{j, j^{\prime}} d B_{j^{\prime}}^{\prime}+\alpha_{j, 0} d T^{\prime} \quad$ for $j \in J, \quad$ and $\quad d T=\sum_{j^{\prime} \in J} \alpha_{0, j^{\prime}} d B_{j^{\prime}}+\alpha_{0,0} d T^{\prime}$, where $\alpha_{j, j^{\prime}} \in \mathbb{O}_{K^{\prime}} \llbracket T \rrbracket$ for $j, j^{\prime} \in J^{+}$. Moreover, we have $\alpha_{0, j} \in T \cdot \mathscr{O}_{K^{\prime}} \llbracket T \rrbracket$. 
We may assume that $\mathscr{E}_{\rho}$ has pure differential nonlog-break, or pure differential log-break. So there exists $\eta_{0} \in(0,1)$ such that $\mathscr{E}_{\rho} \otimes F_{\eta}^{\prime}$ has pure extrinsic (resp. intrinsic) radii $\eta^{b}$ for all $\eta \in\left[\eta_{0}, 1\right)$.

Consider $\eta \in\left(\eta_{0}, 1\right) \cap p^{\mathbb{Q}}$ so that $F_{\eta}^{\prime}$ is discretely valued. Theorem 1.4.20 then implies that, for any $j \in J^{+}$such that $R_{\partial_{j}^{\prime}}\left(V \otimes F_{\eta}^{\prime}\right)=E R\left(V \otimes F_{\eta}^{\prime}\right)$, we have

$$
\begin{aligned}
\Theta_{\partial_{j}^{\prime}}\left(\mathscr{E}_{\rho} \otimes F_{\eta}^{\prime}\right)=\left\{\pi T^{-b}\left(\alpha_{0, j} \theta_{0}+\cdots+\alpha_{m, j} \theta_{m}\right):\right. & \\
& \left.\pi T^{-b}\left(\theta_{0} d T+\theta_{1} d B_{1}+\cdots+\theta_{m} d B_{m}\right) \in \mathscr{E} \Theta\left(\mathscr{E}_{\rho} \otimes F_{\eta}^{\prime}\right)\right\},
\end{aligned}
$$

and for any $j \in J^{+}$such that $I_{\partial_{j}^{\prime}}\left(V \otimes F_{\eta}^{\prime}\right)=I R\left(V \otimes F_{\eta}^{\prime}\right)$,

$$
\begin{aligned}
\Theta_{\partial_{j}^{\prime}}\left(\mathscr{E}_{\rho} \otimes F_{\eta}^{\prime}\right)=\left\{\pi T^{-b}\left(\frac{\alpha_{0, j}}{T} \theta_{0}+\cdots+\alpha_{m, j} \theta_{m}\right):\right. & \\
& \left.\pi T^{-b}\left(\theta_{0} \frac{d T}{T}+\theta_{1} d B_{1}+\cdots+\theta_{m} d B_{m}\right) \in \mathscr{I} \Theta\left(\mathscr{E}_{\rho} \otimes F_{\eta}^{\prime}\right)\right\} .
\end{aligned}
$$

Note also that

$$
\begin{aligned}
\left(\alpha_{0,0} \theta_{0}+\cdots+\alpha_{m, 0} \theta_{m}\right) d T^{\prime}+\sum_{j \in J}\left(\alpha_{0, j} \theta_{0}+\cdots\right. & \left.+\alpha_{m, j} \theta_{m}\right) d B_{j}^{\prime} \\
& =\theta_{0} d T+\theta_{1} d B_{1}+\cdots+\theta_{m} d B_{m} .
\end{aligned}
$$

Combining these two formulas, we conclude that $\mathscr{E} \Theta(V)$ (resp. $\mathscr{I} \Theta(V)$ ) for $\partial_{J^{+}}$ is the same as that for $\partial_{J^{+}}^{\prime}$. Hence the refined Artin and Swan conductors are well-defined.

Lemma 2.3.6. Let $k^{\prime} / k$ be a tamely ramified extension of ramification degree $e=e_{k^{\prime} / k}$ and let $\rho$ be a $p$-adic representation of $G_{k}$ with finite local monodromy and with pure log-break $b=b_{\log }(\rho)$. Then $\left.\rho\right|_{G_{k^{\prime}}}$ has pure log-break eb. Moreover, if we identify $\Omega_{\mathscr{O}_{k}}^{1}(\log ) \otimes_{\mathscr{O}_{k}} \pi_{k}^{-b} \bar{\kappa}$ with $\Omega_{\mathscr{O}_{k^{\prime}}}^{1}(\log ) \otimes_{\mathscr{O}_{k^{\prime}}} \pi_{k^{\prime}}^{-e b} \bar{\kappa}$, then $\operatorname{rsw}(\rho)$ is the same as $\operatorname{rsw}\left(\left.\rho\right|_{g_{k^{\prime}}}\right)$.

Proof. This follows immediately from the fact that $\left.\mathscr{E}^{\mathscr{E}} \rho\right|_{G_{k^{\prime}}}$ is just the base change of $\mathscr{E}_{\rho}$ along $A_{K^{\prime}}^{1}\left[\eta^{1 / e}, 1\right) \rightarrow A_{K^{\prime}}^{1}[\eta, 1)$, where the coordinate for the first annulus is $t^{1 / e}$.

Theorem 2.3.7. Let $k$ be a complete discrete valuation field of equal characteristic $p>0$.

(a) Let $\rho$ be a p-adic representation of $G_{k}$ with finite local monodromy and with pure log-break $b=b_{\log }(\rho)>0$. Then there exists a unique direct sum decomposition of $\rho$ as $\rho \cong \oplus_{\{\vartheta\} \subset \operatorname{rsw}(\rho)} \rho_{\{\vartheta\}}$, where the direct sum is taken over all $\mu_{e} \rtimes G_{k}$-orbits $\{\vartheta\}$ in $\operatorname{rsw}(\rho)$, and $\operatorname{rsw}\left(\rho_{\{\vartheta\}}\right)$ consists of the Galois orbits $\{\vartheta\}$ with appropriate multiplicities. Moreover, there exists a finite tamely ramified extension $k^{\prime} / k$ of naïve ramification degree e such that we have a unique direct sum decomposition of representations of $G_{k^{\prime}}$ over some finite 
extension $F^{\prime}$ of $F:\left.\rho\right|_{G_{k^{\prime}}} \otimes F^{\prime} \cong \bigoplus_{\vartheta \in \operatorname{rsw}(\rho)} \rho_{\vartheta}$, such that $\rho_{\vartheta}$ has pure refined Swan conductors

$$
\vartheta \in \Omega_{\mathscr{O}_{k^{\prime}}}^{1}(\log ) \otimes_{\mathscr{O}_{k^{\prime}}} \pi_{k^{\prime}}^{-e b} \bar{\kappa} \cong \Omega_{\bigcirc_{k}}^{1}(\log ) \otimes_{\mathbb{O}_{k}} \pi_{k}^{-b} \bar{\kappa} .
$$

(b) Choose the p-th root of unity $\zeta_{p}$ as in Remark 2.3.4. Then there exists an injective homomorphism for any $b \in \mathbb{Q}_{>0}$,

$$
\mathrm{rsw}=\operatorname{rsw}_{k}: \operatorname{Hom}\left(\mathrm{Fil}_{\log }^{b} G_{k} / \mathrm{Fil}_{\log }^{b+} G_{k}, \mathbb{F}_{p}\right) \rightarrow \Omega_{\mathscr{O}_{k}}^{1}(\log ) \otimes_{\mathbb{O}_{k}} \pi_{k}^{-b} \bar{\kappa},
$$

such that, when viewing the left hand side as a subset of

$$
\operatorname{Hom}\left(\mathrm{Fil}_{\log }^{b} G_{k} / \mathrm{Fil}_{\log }^{b+} G_{k}, \mathbb{Q}_{p}\left(\zeta_{p}\right)^{\times}\right)
$$

via the identification of $1 \in \mathbb{F}_{p}$ with $\zeta_{p}$, we have, for any $p$-adic representation $\rho$ of $G_{k}$ with finite local monodromy and with pure log-break $b$, the images of the summands of $\left.\rho\right|_{\mathrm{Fil}_{\log }^{b} G_{k}}$ under the homomorphism rsw exactly form the multiset of refined Swan conductors of $\rho$. Moreover, the homomorphism (2.3.8) does not depend on the choices of the Dwork pi.

Proof. For both (a) and (b), we may assume that Hypothesis 2.1.9 holds, since only finitely many elements in a $p$-basis matter.

(a) Using the identification given in Lemma 2.3.6, we may first replace $k$ and Frac 0 by a tamely ramified extension of $k$ and a finite extension of Frac 0 , respectively, so that the decomposition of the $\nabla$-module $\mathscr{E}_{\rho}$ given by (1.5.13) of $\mathscr{E}_{\rho}$ can be realized over $\mathscr{R}_{K^{\prime}}$, and that $\mathbb{F}_{q} \subseteq k_{0}$. Since this decomposition is canonical, it is also a decomposition of $(\phi, \nabla)$-modules. By the slope filtration [Kedlaya 2007, Theorem 3.4.6], the Frobenius action on each direct summand of $\mathscr{E}_{\rho}$ is étale, yielding the decomposition of the representation via the equivalence of categories in Theorem 2.1.7.

(b) The following are immediate corollaries of Proposition 1.3.19.

(i) For any $p$-adic representations $\rho$ and $\rho^{\prime}$ of $G_{k}$ with finite local monodromy, same pure log-break $b$, and same pure refined Swan conductor $\vartheta$, the log-break of $\rho \otimes \rho^{\prime \vee}$ is strictly smaller than $b$.

(ii) For any $p$-adic representations $\rho$ and $\rho^{\prime}$ of $G_{k}$ with finite local monodromy, same pure log-break $b$, but different pure refined Swan conductor $\vartheta \neq \vartheta^{\prime}$, respectively, $\rho \otimes \rho^{\prime \vee}$ has pure log-break $b$ and pure refined Swan conductor $\vartheta-\vartheta^{\prime}$.

We also need the following easy fact about Galois representations.

(iii) For any homomorphism $\chi: \mathrm{Fil}_{\log }^{b} G_{k} / \mathrm{Fil}_{\log }^{b+} G_{k} \rightarrow \mathbb{F}_{p} \hookrightarrow \mathbb{Q}_{p}\left(\zeta_{p}\right)^{\times}$, there exist a finite tamely ramified extension $k^{\prime}$ of $k$ with naïve ramification degree $e$ and a representation $\rho_{\chi}$ of $G_{k^{\prime}}$ with finite local monodromy, pure log-break $e b$, 
and pure refined Swan conductor, such that $\left.\rho_{\chi}\right|_{\mathrm{Fil}_{\log }^{b} G_{k} / \mathrm{Fil}_{\log }^{b+} G_{k}}$ contains $\chi$ as a direct summand.

Proof of (iii): The chosen $p$-th root of unity $\zeta_{p}$ in Remark 2.3.4 promotes $\chi$ to the homomorphism

$$
\chi: \mathrm{Fil}_{\log }^{b} G_{k} / \mathrm{Fil}_{\log }^{b+} G_{k} \rightarrow \mathbb{F}_{p} \rightarrow \mathbb{Q}_{p}\left(\zeta_{p}\right)^{\times}
$$

by identifying 1 with $\zeta_{p}$. Since $G_{k} / \mathrm{Fil}_{\log }^{b+} G_{k}$ is a profinite group, there exists a normal subgroup $H$ of $G_{k}$ of finite index containing Fil $_{\log }^{b+} G_{k}$, such that $\chi$ factors through

$$
I=\mathrm{Fil}_{\log }^{b} G_{k} /\left(H \cap \mathrm{Fil}_{\log }^{b} G_{k}\right) .
$$

Put $\rho^{\prime}=\operatorname{Ind}_{I}^{G_{k} / H} \chi$; then $\left.\rho^{\prime}\right|_{\mathrm{Fil}^{b} G_{k}}$ contains $\chi$ as a direct summand. We may use (a) to write $\left.\rho^{\prime}\right|_{G_{k^{\prime}}}$ for some finite tamely ramified extension $k^{\prime}$ of $k$ as the direct sum of representations with pure refined Swan conductors. Then $\chi$ appears in at least one of the direct summand, which we take to be our chosen $\rho_{\chi}$.

Having established (iii), we define rsw to be the morphism sending $\chi$ to the unique refined Swan conductor of $\rho_{\chi}$, which is an element of

$$
\Omega_{\mathscr{O}_{k^{\prime}}}^{1}(\log ) \otimes_{\mathscr{O}_{k^{\prime}}} \pi_{k^{\prime}}^{-e b} \bar{\kappa} \cong \Omega_{\mathscr{O}_{k}}^{1}(\log ) \otimes_{\mathscr{O}_{k}} \pi_{k}^{-b} \bar{\kappa},
$$

via the identification in Lemma 2.3.6. This map is well-defined by (iv) below and it is clearly a homomorphism. Its injectivity will follow from (v).

(iv) For any two representations $\rho_{\chi}$ and $\rho_{\chi}^{\prime}$ satisfying (iii), they must have the same refined Swan conductor.

Suppose the contrary, that is, $\rho_{\chi}$ and $\rho_{\chi}^{\prime}$ have distinct pure refined Swan conductors $\vartheta$ and $\vartheta^{\prime}$. This in particular implies that $\rho_{\chi} \otimes \rho_{\chi}^{\prime \vee}$ has pure Swan conductor $b$ by (ii). However, the construction of $\rho_{\chi}$ and $\rho_{\chi}^{\prime}$ implies that $\left.\rho_{\chi} \otimes \rho_{\chi}^{\prime \vee}\right|_{G_{k^{\prime}}}$ contains a direct summand trivial on $\mathrm{Fil}_{\log }^{e b} G_{k^{\prime}}$; this is a contradiction.

(v) For two distinct homomorphisms $\chi, \chi^{\prime}: \mathrm{Fil}_{\log }^{b} G_{k} / \mathrm{Fil}_{\log }^{b+} G_{k} \rightarrow \mathbb{F}_{p}$, the representations $\rho_{\chi}$ and $\rho_{\chi^{\prime}}$ given by (iii) have distinct refined Swan conductors.

Suppose the contrary. Then (i) implies that $\rho_{\chi} \otimes \rho_{\chi^{\prime}}^{\vee}$ would have log-break strictly less than $e b$. However, $\rho_{\chi} \otimes \rho_{\chi^{\prime}}^{\vee}$, when restricted to

$$
\mathrm{Fil}_{\log }^{b} G_{k} / \mathrm{Fil}_{\log }^{b+} G_{k}=\mathrm{Fil}_{\log }^{e b} G_{k^{\prime}} / \mathrm{Fil}_{\log }^{e b+} G_{k^{\prime}}
$$

has a direct summand isomorphic to $\chi \otimes \chi^{\prime \vee}$, which is nontrivial. This is a contradiction.

We now prove the independence on the choice of the Dwork pi. If we choose another Dwork pi, we would need to use another primitive $p$-th root of unity $\zeta_{p}^{i}$ for 
some $i \in 1, \ldots, p-1$. On one hand, the refined Swan conductor is multiplied by $\left(\zeta_{p}^{i}-1\right) /\left(\zeta_{p}-1\right) \equiv i \bmod \left(\zeta_{p}-1\right)$. On the other hand, the $p$-adic representation

$$
\mathrm{Fil}_{\log }^{b} G_{k} / \mathrm{Fil}_{\log }^{b+} G_{k} \rightarrow \mathbb{Q}_{p}\left(\zeta_{p}\right)^{\times}
$$

becomes $\chi^{i}$. Hence we need to take $\rho_{\chi}^{\otimes i}$ as our $p$-adic representation of $G_{k^{\prime}}$ to define the homomorphism rsw. This representation has refined Swan conductor $\operatorname{rsw}\left(\rho_{\chi}^{\otimes i}\right)=i \cdot \operatorname{rsw}\left(\rho_{\chi}\right)$, which is the same as the refined Swan conductor of $\rho$ computed using the old Dwork pi.

Remark 2.3.9. It is interesting to point out that the choice of a Dwork pi is related to the choice of the Artin-Scheier $\ell$-adic sheaf in [Saito 2009]; they both amount to choosing a primitive $p$-th root of unity. The difference is that we consider it as an element in $\overline{\mathbb{Q}}_{p}$ whereas Saito viewed it as an element in $\overline{\mathbb{Q}}_{l}$.

Proposition 2.3.10. Let $k$ be a complete discrete valuation field of equal characteristic $p>0$. Then for $b \in \mathbb{Q}_{>0}$, the conjugation action of

$$
\mathrm{Fil}_{\log }^{0+} G_{k} / \mathrm{Fil}_{\log }^{b} G_{k} \quad \text { on } \mathrm{Fil}_{\log }^{b} G_{k} / \mathrm{Fil}_{\log }^{b+} G_{k}
$$

is trivial. In other words, $\mathrm{Fil}_{\log }^{b} G_{k} / \mathrm{Fil}_{\log }^{b+} G_{k}$ lies in the center of $\mathrm{Fil}_{\log }^{0+} G_{k} / \mathrm{Fil}_{\log }^{b+} G_{k}$. Proof. This proposition is proved in [Abbes and Saito 2003, Theorem 1]. We give an alternative proof using differential modules.

It suffices to prove the following: for a $p$-adic representation $\rho$ of $G_{k}$ with finite local monodromy and with pure $\log$-break $b$, if it is absolutely irreducible under any tamely ramified extension, then

$$
\left.\rho\right|_{\mathrm{Fil}_{\log }^{b} G_{k} / \mathrm{Fil}_{\log }^{b+} G_{k}}
$$

is a direct sum of a single character $\chi: \mathrm{Fil}_{\log }^{b} G_{k} / \mathrm{Fil}_{\log }^{b+} G_{k} \rightarrow \mathrm{O}^{\times}$. This is equivalent to showing that the action of $\mathrm{Fil}_{\log }^{b} G_{k}$ on $\rho \otimes \rho^{\vee}$ is trivial, and hence to showing that the log-break of $\rho \otimes \rho^{\vee}$ is strictly smaller than $b$.

As usual, we may assume Hypothesis 2.1.9. By Theorem 2.3.7(a), the irreducibility condition on $\rho$ implies that $\rho$ must have pure refined Swan conductor and hence the log-break $\rho \otimes \rho^{\vee}$ must be strictly less than $b$. We are done.

Proposition 2.3.11. Keep the notation as in Situation 2.2.13. Then the refined Swan conductor homomorphism $\mathrm{rsw}_{k}$ for $k$ factors as

$$
\begin{aligned}
\operatorname{Hom}\left(\mathrm{Fil}_{\log }^{b} G_{k} / \mathrm{Fil}_{\log }^{b+} G_{k}, \mathbb{F}_{p}\right) \longrightarrow \operatorname{Hom}\left(\mathrm{Fil}_{\log }^{e_{\tilde{k} / k}^{b}} G_{\tilde{k}} / \mathrm{Fil}_{\log }^{e_{\tilde{k} / /}^{b+}} G_{\tilde{k}}, \mathbb{F}_{p}\right) \\
\stackrel{\operatorname{rsw}_{\tilde{k}}}{\longrightarrow} \Omega_{\mathscr{C}_{\tilde{k}}}^{1}(\log ) \otimes_{\mathscr{O}_{\tilde{k}}} \pi_{\tilde{k}}^{-e b} \kappa_{\tilde{k}} \mathrm{alg} .
\end{aligned}
$$

Proof. Keep the notation as in Proposition 2.2.14, let $\widetilde{F}_{\eta}^{\prime}$ be the completion of $\widetilde{K}^{\prime}(U)$ with respect to the $\eta^{1 / e}$-Gauss norm in $U$. Fix $\eta_{0} \in(0,1)$ such that $I R\left(\mathscr{E}_{\rho} \otimes F_{\eta}^{\prime}\right)=\eta^{b}$ 
for $\eta \in\left[\eta_{0}, 1\right)$. Then (2.2.15) implies that, for any $\eta \in\left[\eta_{0}, 1\right) \cap p^{\mathbb{Q}}$ and for any $j \in\{0, \ldots, m+1\}$ such that $I R_{\partial_{j}}\left(f^{* \mathscr{E}_{\rho}} \otimes \widetilde{F}_{\eta}^{\prime}\right)=\operatorname{IR}\left(\mathscr{E}_{\rho} \otimes \widetilde{F}_{\eta}^{\prime}\right)$, we have

$$
\Theta_{\partial_{j}}\left(f^{* \mathscr{E}} \mathscr{E}_{\rho} \otimes \widetilde{F}_{\eta}^{\prime}\right)= \begin{cases}\Theta_{\partial_{j}}\left(\mathscr{E} \otimes F_{\eta}^{\prime}\right) & \text { if } j \in J, \\ e X U^{e-1} \Theta_{\partial_{0}}\left(\mathscr{E} \otimes F_{\eta}^{\prime}\right) & \text { if } j=0 \text { and hence } p \nmid e, \\ U^{e} \Theta_{\partial_{m+1}}\left(\mathscr{E} \otimes F_{\eta}^{\prime}\right) & \text { if } j=m+1,\end{cases}
$$

using Theorem 1.4.20 to compute the refined radii. The proposition follows.

One may want to prove analogs of Theorem 2.3.7 and Proposition 2.3.10 for refined Artin conductors. This however needs to take a bit more effort because there may not be a representation of $G_{k}$ with pure refined Artin conductor. Instead, we reduce to the classical case, where the results for refined Artin conductors follows from those for refined Swan conductors.

Theorem 2.3.13. Let $k$ be a complete discrete valuation field of equal characteristic $p>0$.

(a) Choose the p-th root of unity $\zeta_{p}$ as in Remark 2.3.4. Then there exists an injective homomorphism for any $b \in \mathbb{Q}_{>1}$,

$$
\operatorname{rar}=\operatorname{rar}_{k}: \operatorname{Hom}\left(\mathrm{Fil}^{b} G_{k} / \mathrm{Fil}^{b+} G_{k}, \mathbb{F}_{p}\right) \rightarrow \Omega_{\mathscr{O}_{k}}^{1} \otimes_{\mathscr{O}_{k}} \pi_{k}^{-b} \bar{\kappa},
$$

such that, when viewing the left hand side as a subset of

$$
\operatorname{Hom}\left(\mathrm{Fil}^{b} G_{k} / \mathrm{Fil}^{b+} G_{k}, \mathbb{Q}_{p}\left(\zeta_{p}\right)^{\times}\right)
$$

via the identification of $1 \in \mathbb{F}_{p}$ with $\zeta_{p}$, we have, for any $p$-adic representation $\rho$ of $G_{k}$ with finite local monodromy and with pure nonlog-break $b$, the images of the summands of $\left.\rho\right|_{\mathrm{Fil}^{b} G_{k}}$ under rar exactly form the multiset of refined Artin conductors of $\rho$. Moreover, this homomorphism does not depend on the choices of the Dwork pi.

(b) For any $b \in \mathbb{Q}_{>1}$, the conjugation action of

$$
\mathrm{Fil}^{1+} G_{k} / \mathrm{Fil}^{b} G_{k} \quad \text { on } \mathrm{Fil}^{b} G_{k} / \mathrm{Fil}^{b+} G_{k}
$$

is trivial. That is, $\mathrm{Fil}^{b} G_{k} / \mathrm{Fil}^{b+} G_{k}$ lies in the center of $\mathrm{Fil}^{1+} G_{k} / \mathrm{Fil}^{b+} G_{k}$.

Proof. For both (a) and (b), we may assume Hypothesis 2.1.9. Moreover, we assume that $J$ is not empty because otherwise we are in the classical case, and both (a) and (b) follow from their log-version counterpart: Theorem 2.3.7 and Proposition 2.3.10, respectively.

We perform a base change similar to the one in Lemma 2.2.9. Let $k^{\prime}$ be the completion of $k\left(x_{1}, \ldots, x_{m}\right)$ with respect to the $(1, \ldots, 1)$-Gauss norm and let $\tilde{k}$ be the completion of

$$
k^{\prime}\left(\left(b_{j}+x_{j} \pi_{k}\right)^{1 / p^{n}}, x_{j}^{1 / p^{n}} ; n \in \mathbb{N} ; j \in J\right)
$$


equipped with the uniformizer $\pi_{\tilde{k}}=\pi_{k}$. It is in fact a complete discrete valuation field with perfect residue field. By Lemmas 2.2.9 and 2.2.11, the natural homomorphism $G_{\tilde{k}} \rightarrow G_{k}$ induces a surjective homomorphism

$$
\mathrm{Fil}^{a} G_{\tilde{k}} / \mathrm{Fil}^{a+} G_{\tilde{k}} \longrightarrow \mathrm{Fil}^{a} G_{k} / \mathrm{Fil}^{a+} G_{k} .
$$

Dualizing this gives an injective homomorphism

$$
\mu: \operatorname{Hom}\left(\mathrm{Fil}^{a} G_{k} / \mathrm{Fil}^{a+} G_{k}, \mathbb{F}_{p}\right) \rightarrow \operatorname{Hom}\left(\mathrm{Fil}^{a} G_{\tilde{k}} / \mathrm{Fil}^{a+} G_{\tilde{k}}, \mathbb{F}_{p}\right) .
$$

For $\rho$ a representation of $G_{k}$ with finite local monodromy and with pure nonlogbreak $b$ we let $\tilde{\rho}$ denote the representation $G_{\tilde{k}} \rightarrow G_{k} \stackrel{\rho}{\rightarrow} G L\left(V_{\rho}\right)$. Let $K^{\prime \prime}$ denote the completion of $K^{\prime}\left(X_{J}\right)$ with respect to the $(1, \ldots, 1)$-Gauss norm, where $X_{j}$ is a lift of $x_{j}$ for $j \in J$. Let $\widetilde{K}$ denote the completion of

$$
K^{\prime \prime}\left(\left(B_{j}+X_{j} T\right)^{1 / p^{n}}, X_{j}^{1 / p^{n}} ; n \in \mathbb{N}, j \in J\right) .
$$

Let $f: A_{\widetilde{K}}^{1}\left[\eta_{0}, 1\right) \rightarrow A_{K^{\prime}}^{1}\left[\eta_{0}, 1\right)$ denote the natural morphism. Then $f^{* \mathscr{E}_{\rho}}$ is the differential module associated to $\tilde{\rho}$. Let $\tilde{\partial}$ denote the differential operator on $f^{* \mathscr{E}} \rho$ dual to the basis $d T$. Similar to (2.2.10), we have

$$
\tilde{\partial}=\partial_{0}-X_{1} \partial_{1}-\cdots-X_{m} \partial_{m} .
$$

If we let $\widetilde{F}_{\eta}$ denote the completion of $\widetilde{K}(T)$ with respect to the $\eta$-Gauss norm, we have

$$
R_{\tilde{\partial}}\left(f^{* \mathscr{E}} \otimes \widetilde{F}_{\eta}\right)=\min _{j \in J^{+}}\left\{R_{\partial_{j}}\left(\mathscr{E} \otimes F_{\eta}^{\prime}\right)\right\} .
$$

Hence $\tilde{\rho}$ has pure nonlog-break $b$ and, by Theorem 1.4.20, its multiset of refined Artin conductors is

$\operatorname{rar}(\tilde{\rho})=\left\{\left(\theta_{0}-X_{1} \theta_{1}-\cdots-X_{m} \theta_{m}\right) d \pi_{k} \mid \theta_{0} d \pi_{k}+\theta_{1} d b_{1}+\cdots+\theta_{m} d b_{m} \in \operatorname{rar}(\rho)\right\}$.

In other words, if we consider the $\bar{\kappa}$-linear injective homomorphism

$$
\lambda: \Omega_{\mathscr{O}_{k}}^{1} \otimes_{\mathscr{O}_{k}} \pi_{k}^{-b} \bar{\kappa} \rightarrow \pi_{k}^{-b} \kappa_{\tilde{k}^{\mathrm{alg}}} d \pi_{k}
$$

given by $\lambda\left(d b_{j}\right)=-X_{j} d \pi_{k}$ and $\lambda\left(d \pi_{k}\right)=d \pi_{k}$, then $\operatorname{rar}(\tilde{\rho})=\lambda(\operatorname{rar}(\rho))$. This together with the injectivity of $\mu$ reduce (a) and (b) for $G_{k}$ to that of $G_{\tilde{k}}$, which is already known as we explained earlier. In particular, we have $\lambda \circ \operatorname{rsw}_{k}=\operatorname{rsw}_{\tilde{k}} \circ \mu$.

2.4. Multi-indexed ramification filtrations for higher local fields. When $k$ is an $n$-dimensional local field, the refined Artin and Swan conductors give more refined filtrations on the Galois group $G_{k}$, indexed by $\mathbb{Q}^{n}$ with lexicographic order. We restrict ourselves to the equal characteristic $p>0$ case. 
Definition 2.4.1. We say that a complete discrete valuation field $k$ of characteristic $p>0$ is an $(m+1)$-dimensional local field if there is a chain of fields $k=k_{m+1}, k_{m}, \ldots, k_{0}$, where $k_{i+1}$ is a complete discrete valuation field with residue field $k_{i}$ for $i=0, \ldots, m$. Contrary to most literature, we do not assume that $k_{0}$ is a perfect field. Let $\left\{b_{j}\right\}_{j \in J}$ be a set of lifts of a $p$-basis of $k_{0}$ to $\mathscr{O}_{k}$.

An $(m+1)$-tuple of elements $t_{0}, \ldots, t_{m} \in k$ is called a system of local parameters of $k$ if $t_{i} \in \mathbb{O}_{k}$ is a lift of a uniformizer of $k_{m+1-i}$ all the way up to $k$. Such a choice gives a (noncanonical) isomorphism $k \simeq k_{0}\left(\left(t_{m}\right)\right) \cdots\left(\left(t_{0}\right)\right)$. In this case, we have

$$
\Omega_{\mathscr{O}_{k^{\prime}}}^{1}(\log )=\bigoplus_{i=0}^{m} \mathcal{O}_{k^{\prime}} \frac{d t_{i}}{t_{i}} \oplus \bigoplus_{j \in J} \mathcal{O}_{k^{\prime}} \frac{d b_{j}}{b_{j}} \quad \text { and } \quad \Omega_{\mathscr{O}_{k^{\prime}}}^{1}=\bigoplus_{i=0}^{m} \mathcal{O}_{k^{\prime}} d t_{i} \oplus \bigoplus_{j \in J} \mathcal{O}_{k^{\prime}} d b_{j}
$$

Equip $\mathbb{Q}^{m+1}$ with the lexicographic order: $\boldsymbol{i}=\left(i_{1}, \ldots, i_{m+1}\right)<\boldsymbol{j}=\left(j_{1}, \ldots, j_{m+1}\right)$ if and only if, for some $l \leq m+1$,

$$
i_{l}<j_{l}, \quad i_{l+1}=j_{l+1}, \quad \ldots, \quad i_{m+1}=j_{m+1} .
$$

For $a \in \mathbb{Q}$, we use $\mathbb{Q}_{>a}^{m+1}$ to denote the subset of $\mathbb{Q}^{m+1}$ consisting of $\boldsymbol{i}=\left(i_{1}, \ldots, i_{m+1}\right)$ such that $i_{m+1}>a$.

Given a system of local parameters, we define a multi-indexed valuation as follows, denoted by $\boldsymbol{v}=\left(v_{1}, \ldots, v_{m+1}\right): k^{\times} \rightarrow \mathbb{Z}^{m+1} \subset \mathbb{Q}^{m+1}$, where $v_{m+1}=v_{k_{m+1}}$ and recursively we have, downwards from $i=m+1$ to $i=1$, that $v_{i-1}(\alpha)=$ $v_{k_{i-1}}\left(\alpha_{i-1}\right)$ with $\alpha_{i-1}$ equal to the reduction of $\alpha_{i} t_{m+1-i}^{-v_{i}\left(\alpha_{i}\right)}$ in $k_{i-1}$. Note that the definition of $\boldsymbol{v}$ depends on the choice of local parameters $t_{0}, \ldots, t_{m}$.

Definition 2.4.2. For $\lambda=\sum_{i=0}^{m} \alpha_{i} d t_{i}+\sum_{j \in J} \beta_{j} d b_{j} \in \Omega_{\mathscr{O}_{k}}^{1} \otimes_{\mathfrak{O}_{k}} k$, we set

$$
\boldsymbol{v}_{\mathrm{n} \log }(\lambda)=\min \left\{\boldsymbol{v}\left(\alpha_{0}\right), \ldots, \boldsymbol{v}\left(\alpha_{m}\right), \boldsymbol{v}\left(\beta_{j}\right) ; j \in J\right\} .
$$

This gives a multi-indexed valuation on $\Omega_{\mathscr{O}_{k}}^{1} \otimes_{\mathscr{O}_{k}} t_{0}^{-i_{m+1}} \bar{\kappa}$ for $i_{m+1} \in \mathbb{Q}$.

For $\lambda=\sum_{i=0}^{m} \alpha_{i} \frac{d t_{i}}{t_{i}}+\sum_{j \in J} \beta_{j} \frac{d b_{j}}{b_{j}} \in \Omega_{\mathscr{O}_{k}}^{1}(\log ) \otimes_{\mathfrak{O}_{k}} k$, we set

$$
\boldsymbol{v}_{\log }(\lambda)=\min \left\{\boldsymbol{v}\left(\alpha_{0}\right), \ldots, \boldsymbol{v}\left(\alpha_{m}\right), \boldsymbol{v}\left(\beta_{j}\right) ; j \in J\right\} .
$$

This gives a multi-indexed valuation on $\Omega_{\mathscr{O}_{k}}^{1}(\log ) \otimes_{\mathscr{C}_{k}} t_{0}^{-i_{m+1}} \bar{\kappa}$ for $i_{m+1} \in \mathbb{Q}$.

For $\boldsymbol{i}=\left(i_{1}, \ldots, i_{m+1}\right) \in \mathbb{Q}_{>1}^{m+1}$, we define Fil ${ }^{i} G_{k}$ to be the subgroup of $\mathrm{Fil}^{i_{m+1}} G_{k}$ given by the intersection of the kernels of characters

$$
\chi: \mathrm{Fil}^{i_{m+1}} G_{k} \rightarrow \mathrm{Fil}^{i_{m+1}} G_{k} / \mathrm{Fil}^{i_{m+1}+} G_{k} \rightarrow \mathbb{F}_{p}
$$

for which $\boldsymbol{v}_{\mathrm{nlog}}(\chi)>-\boldsymbol{i}$. We similarly define Fil ${ }^{i} G_{k}$ for $\boldsymbol{i}=\left(i_{1}, \ldots, i_{m+1}\right) \in \mathbb{Q}_{>0}^{m+1}$ by adding subscripts log to the definition above. 
Remark 2.4.3. The abstract filtrations do not depend on the choices of local parameters, but the indexings do. Set $O_{K}=\{x \in K: \boldsymbol{v}(x) \geq(0, \ldots, 0)\}$. It might be more natural to index the above filtrations by "rational powers of fractional ideals of $K$ " of the form $I^{1 / n}$, where $I$ is an $O_{K}$-submodule of $K$ containing $O_{K}, n$ is an integer, and $I^{1 / n}$ is equivalent to $I^{\prime 1 / n^{\prime}}$ if $I^{n^{\prime}}=I^{\prime n}$ as $O_{K}$-submodules of $K$.

Remark 2.4.4. When $k_{0}$ is a finite field, this filtration is expected to be compatible with an easily defined filtration on the Milnor $K$-groups via class field theory for higher local fields. This may be verified by comparing the filtration on the Milnor K-groups with Kato's refined Swan conductors, which is equivalent to Saito's definition by [Abbes and Saito 2009, Theorem 9.1.1] and hence to our definition by Theorem 3.4.1 proved later. For more along this line, the reader may refer to the recipe in Kato's masterpiece [Kato 1989].

\section{Comparison with Saito's definition}

In this section, we compare our definition of the refined Swan conductor homomorphism with the one given by Saito in [Saito 2009]. Since the reader who is only interested in one side of the story may use this result (Theorem 3.4.1) as a black box, we present the proof assuming that the reader is familiar with the definition of arithmetic ramification filtrations; see for instance [Saito 2009, Section 1; Xiao 2010].

The proof of the comparison theorem is of a geometric nature. We explain the rough idea here. We first realize the given finite extension $l$ of $k$ as the corresponding extension of function fields of a finite étale extension of smooth affine varieties $Y \rightarrow X$. Our main object is some version of infinitesimal neighborhood of the generic fiber over $k$ of the diagonal embedding of $Y$ into $Y \times Y$, viewed as a rigid analytic space over $k$. The refined Swan conductor homomorphism defined by Saito makes use of the stable formal model of such an object, whereas our definition using differential modules is closely related to some object over the generic point of a smooth model over $O_{K}$ lifting the aforementioned rigid space. The crucial calculation we performed in Section 3.3 relates these objects, in which case it boils down to some explicit computation on a higher dimensional analog of the Artin-Scheier cover, and on the associated $\ell$-adic sheaves and overconvergent $F$-isocrystals.

We assume $p>0$ is a prime number throughout this section.

3.1. Review of Saito's definition. In this subsection, we review the definition of the ramification filtrations and the refined Swan conductors defined by Abbes and Saito in [Abbes and Saito 2002; 2003; Saito 2009]. Instead of introducing the general construction, we will focus on a special case which is used in the comparison theorem. For more details and a complete treatment, one may consult [Saito 2009]. 
Construction 3.1.1. Let $l$ be a finite Galois extension of $k$. We consider a closed immersion $\mathrm{Spec} \mathrm{O}_{l} \rightarrow P$ into a smooth (affine) scheme $P$ over Spec $\mathrm{O}_{k}$. Put $\mathscr{I}=\operatorname{Ker}\left(\mathrm{O}_{P} \rightarrow \mathrm{O}_{l}\right)$.

For $r=a / b \in \mathbb{Q}$ with $a, b>0$, let $P_{\mathscr{O}_{k}}^{[a / b]} \rightarrow P$ be the blowup at the ideal $g^{b}+\mathfrak{m}_{k}^{a} \mathcal{O}_{P}$ and let $P_{\mathscr{O}_{k}}^{(a / b)} \subset P_{\mathscr{O}_{k}}^{[a / b]}$ be the complement of the support of

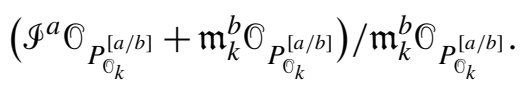

Let $P_{\widehat{O}_{k}}^{(r)}$ be the normalization of $P_{\bigcirc_{k}}^{(a / b)}$; it does not depend on $a$ and $b$ but only on their ratio. Let $P_{k}^{(r)}$ and $P_{\kappa}^{(r)}$ denote the generic fiber and the special fiber of $P_{\mathscr{O}_{k}}^{(r)}$, respectively. Let $\widehat{P}_{k}^{(r)}$ denote the generic fiber of completing $P_{\mathbb{O}_{k}}^{(r)}$ along $P_{\kappa}^{(r)}$. The immersion $\mathrm{Spec} \mathrm{O}_{l} \rightarrow P$ is uniquely lifted to an immersion Spec $\mathrm{O}_{l} \rightarrow P_{\mathscr{O}_{k}}^{(r)}$.

By the finiteness theorem of Grauert-Remmert cited in [Abbes and Saito 2003, Theorem 1.10], there exists a finite separable extension $k^{\prime} / k$ of naïve ramification degree $e=e_{k^{\prime} / k}$ such that the normalization $P_{\mathscr{O}_{k^{\prime}}}^{(e r)}$ of $P_{\mathscr{O}_{k}}^{(r)} \times_{\mathscr{O}_{k}} \mathscr{O}_{k^{\prime}}$ has reduced geometric fibers over $\operatorname{Spec} \mathbb{O}_{k^{\prime}}$, which we call a stable model of $P_{\mathscr{O}_{k}}^{(r)}$. We put

$$
P_{\bar{\kappa}}^{(r)}=P_{\mathscr{O}_{k^{\prime}}}^{(e r)} \times_{\mathscr{O}_{k^{\prime}}} \bar{\kappa}
$$

this is called the stable special fiber of $P_{\mathscr{O}_{k}}^{(r)}$ and it does not depend on the choice of $k^{\prime}$.

We defer the discussion of the properties of this construction until later when we have a concrete example at hand.

For the rest of this section, we make the following geometric assumption.

Hypothesis 3.1.2 (Geom). There exists an affine smooth variety $X$ over $k_{0}$ and an irreducible divisor $D$, smooth over $k_{0}$ with generic point $\xi$, such that $\mathrm{O}_{k} \cong 0_{X, \xi}^{\wedge}$, where the latter is the completion of the local ring at $\xi$. In particular, Hypothesis 2.1.9 is fulfilled.

Remark 3.1.3. This hypothesis is essentially the same as the hypothesis of the same name in [Saito 2009, p. 786], except that our $k$ is the completion of the Henselian local field considered in Saito's paper.

Construction 3.1.4. After replacing $X$ (and hence $D$ ) by an étale neighborhood of $\xi$ if necessary, there exists a finite flat morphism $f: Y \rightarrow X$ of smooth varieties over $k_{0}$ such that $V=Y \times_{X} U \rightarrow U=X \backslash D$ is finite étale with Galois group $G_{l / k}$ and that $Y \times{ }_{X} \operatorname{Spec}^{\wedge}{ }_{X, \xi}=\operatorname{Spec} O_{l}$.

Let $(X \times X)^{\prime}$ be the blowup of $X \times_{k_{0}} X$ along $D \times_{k_{0}} D$, and let $(X \times X)^{\sim}$ denote the complement of the proper transforms of $X \times_{k_{0}} D$ and $D \times_{k_{0}} X$ in $(X \times X)^{\prime}$. The diagonal embedding $\Delta_{X}: X \rightarrow X \times_{k_{0}} X$ naturally lifts to an embedding $\tilde{\Delta}_{X}: X \rightarrow(X \times X)^{\sim}$. Now, pulling back the whole picture along $f: Y \rightarrow X$ gives 
the commutative diagram

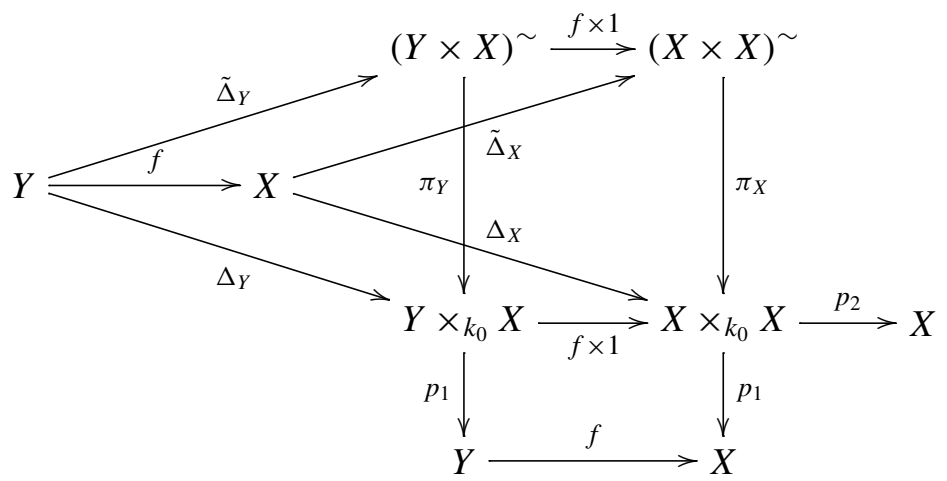

where $(Y \times X)^{\sim}$ is the fiber product of the big square, and all parallelograms are Cartesian.

Put $P=(X \times X)^{\sim} \times_{p_{2} \circ \pi_{X}, X} \operatorname{Spec} \mathcal{O}_{X, \xi}^{\wedge} \quad$ and $\quad Q=(Y \times X)^{\sim} \times_{p_{2} \circ \pi_{X} \circ(f \times 1), X} \operatorname{Spec} \mathcal{O}_{X, \xi}^{\wedge}$. Taking the Cartesian product of the top part of (3.1.5) with $\operatorname{Spec}^{\wedge}{ }_{X, \xi}=\operatorname{Spec} O_{k}$ over $X \times_{k_{0}} X$ along $p_{2}$ then gives the following commutative diagram.

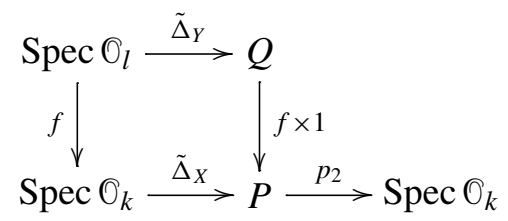

Let $\Phi$ denote the ideal of the immersion $\tilde{\Delta}_{X}$. We will view $P$ and $Q$ as schemes over $\mathbb{O}_{k}$ via $p_{2}$.

We can now apply Construction 3.1.1 to the embeddings $\tilde{\Delta}_{X}$ and $\tilde{\Delta}_{Y}$ to define

$$
P_{O_{k}^{\prime}}^{(e r)}, P_{k^{\prime}}^{(e r)}, \widehat{P}_{k^{\prime}}^{(e r)}, P_{\bar{\kappa}}^{(e r)} \quad \text { and } \quad Q_{{O_{k}^{\prime}}^{\prime}}^{(e r)}, Q_{k^{\prime}}^{(e r)}, \widehat{Q}_{k^{\prime}}^{(e r)}, Q_{\bar{\kappa}}^{(e r)},
$$

respectively, where $k^{\prime} / k$ is a finite separable extension of naïve ramification degree $e$. We still use $p_{1}$ to denote the morphism $P_{\mathscr{O}_{k^{\prime}}}^{(e r)} \rightarrow P \stackrel{p_{1}}{\rightarrow}$ Spec $\mathscr{O}_{k}$. By functoriality of Construction 3.1.1, we have a morphism $f^{(r)}: Q_{\mathscr{O}_{k^{\prime}}}^{(e r)} \rightarrow P_{\mathscr{C}_{k^{\prime}}}^{(e r)}$.

Remark 3.1.7. The field extension $k^{\prime}$ serves as the role of a "coefficient field"; we only use it to provide reasonable integral structures of our spaces over $\mathrm{O}_{k^{\prime}}$, and also to make $e r$ an integer. We can make $k^{\prime}$ as large as we need.

In contrast, the extension $l / k$ pulled back from $p_{1}: X \times_{k_{0}} X \rightarrow X$ encodes the arithmetic information.

We collect together some properties of these spaces. 
Proposition 3.1.8. Let $k^{\prime} / k$ be a finite separable extension of nä̈ve ramification degree e.

(a) When er is an integer, the space $P_{\mathscr{O}_{k^{\prime}}}^{(e r)}$ is defined to be $\sum_{i \geq 0} \pi_{k^{\prime}}^{-i e r}$. $g^{i} \subset \mathscr{O}_{P} \otimes_{\mathscr{O}_{k}} k^{\prime}$. It is smooth over $O_{k^{\prime}}$, and its closed fiber $P_{\kappa_{k^{\prime}}}^{(e r)}$ can be canonically identified with the $\kappa_{k^{\prime}}$-vector space $\Omega_{\mathscr{O}_{k}}^{1}(\log ) \otimes_{\mathscr{O}_{k}} \pi_{k^{\prime}}^{-e r} \kappa_{k^{\prime}}$. The rigid space

$$
\widehat{P}_{k^{\prime}}^{(e r)} \quad \text { is isomorphic to } \operatorname{Sp}\left(k^{\prime}\left\langle\pi_{k^{\prime}}^{-e r-e} \delta_{0}, \pi_{k^{\prime}}^{-e r} \delta_{J}\right\rangle\right),
$$

where $\delta_{0}, \ldots, \delta_{m}$ form a dual basis of $\Omega_{\mathscr{O}_{k}}^{1}$.

(b) The generic fiber $Q_{k^{\prime}}^{(e r)}$ of $Q_{\widehat{O}_{k^{\prime}}}^{(e r)}$ is isomorphic to $P_{k^{\prime}}^{(e r)} \otimes_{p_{1}, k}$ l. In particular, $Q_{k^{\prime}}^{(e r)}$ is finite and étale over $P_{k^{\prime}}^{(e r)}$ with Galois group $G_{l / k}$, and the same is true for

$$
\widehat{Q}_{k^{\prime}}^{(e r)} \text { over } \widehat{P}_{k^{\prime}}^{(e r)} \text {. }
$$

(c) Let $\mathrm{Spf}^{0} Q^{\wedge}$ be the completion of $Q$ along Spec $\mathcal{O}_{l}$. If er is an integer, then

$$
\widehat{Q}_{k^{\prime}}^{(e r)} \text { is the affinoid variety } X_{\log }^{j}\left(\mathscr{O}_{Q^{\wedge}} \rightarrow \mathscr{O}_{l}\right)_{k^{\prime}}
$$

defined in [Abbes and Saito 2003, Section 4.2] for $j=r$.

(d) If the highest log-break $b_{\log }(l / k)$ is less than or equal to $r$, then $Q_{\bar{\kappa}}^{(r)}$ is an element of the category $\left(\mathrm{FE} / P_{\bar{\kappa}}^{(r)}\right)^{\mathrm{alg}}$, defined below in Definition 3.1.9.

(e) The highest log-break $b_{\log }(l / k)$ is strictly less than $r$ if and only if the number of connected components of $Q_{\bar{\kappa}}^{(r)}$ is $[l: k]$.

Proof. For (a), see [Saito 2009, Lemma 1.10]. The claim (b) follows from the fact that $f: V \rightarrow U$ is finite and étale with Galois group $G_{l / k}$. For (c), see [ibid., Example 1.21]. The statements (d) and (e) follow from [ibid., Lemma 1.13 and Theorem 1.24].

Definition 3.1.9. For an $\bar{\kappa}$-vector space $W$ of finite dimensional, let $(\mathrm{FE} / W)^{\text {alg }}$ be the full subcategory of (FE/ $W$ ) whose objects are finite étale morphisms $g: Z \rightarrow W$ such that $Z$ admits a structure of algebraic group scheme and such that $g$ is a morphism of algebraic groups.

Remark 3.1.10. By the argument just before [ibid., Lemma 1.23], the category $(\mathrm{FE} / W)^{\text {alg }}$ is a Galois category associated to the Galois group $\pi_{1}^{\text {alg }}(W)$, which is a quotient of the fundamental group $\pi_{1}(W)$. This group can be identified with the Pontrjagin dual of the extension group $\operatorname{Ext}^{1}\left(W, \mathbb{F}_{p}\right)$ in the category of smooth algebraic groups over $\bar{\kappa}$. The map $W^{\vee}=\operatorname{Hom}_{\bar{\kappa}}(W, \bar{\kappa}) \rightarrow \operatorname{Ext}^{1}\left(W, \mathbb{F}_{p}\right)$ sending a linear form $f: W \rightarrow \mathbb{A}_{\bar{\kappa}}^{1}$ to the pullback along $f$ of the Artin-Scheier sequence

$$
0 \rightarrow \mathbb{F}_{p} \rightarrow \mathbb{A}_{\bar{\kappa}} \stackrel{\frac{t \rightarrow t^{p}-t}{\longrightarrow}}{\longrightarrow} \mathbb{A}_{\bar{\kappa}}^{1} \rightarrow 0
$$


is an isomorphism.

Proposition 3.1.11. We have a surjective homomorphism

$$
\pi_{1}^{\mathrm{alg}}\left(P_{\bar{\kappa}}^{(b)}\right) \rightarrow \mathrm{Fil}_{\log }^{b} G_{k} / \mathrm{Fil}_{\log }^{b+} G_{k} ;
$$

it induces an injective homomorphism

$$
\mathrm{rsw}^{\prime}: \operatorname{Hom}\left(\mathrm{Fil}_{\log }^{b} G_{k} / \mathrm{Fil}_{\log }^{b+} G_{k}, \mathbb{F}_{p}\right) \longrightarrow \Omega_{\mathscr{O}_{k}}^{1}(\log ) \otimes_{\mathfrak{O}_{k}} \pi_{k}^{-b} \bar{\kappa} .
$$

Proof. For the first half of the proposition, see [Saito 2009, Theorem 1.24]. The second half follows from Remark 3.1.10.

In the following special case, we give a more detailed description of these spaces.

Situation 3.1.12. Let $l / k$ be a finite totally ramified Galois extension, which is not tamely ramified. Assume that the highest log-break $b=b_{\log }(l / k)$ is a positive integer. Assume moreover that $\mathrm{Fil}_{\log }^{b-1} G_{k} /\left(\mathrm{Fil}_{\log }^{b-1} G_{k} \cap G_{l}\right) \simeq \mathbb{F}_{p}$; in particular, the second highest log-break $b_{\log }(l / k ; 2)$ is strictly less than $b_{\log }(l / k)-1$. By Proposition 3.1.11, $Q_{\bar{\kappa}}^{(b)}$ consists of $[l: k] / p$ copies of the same Artin-Scheier cover of $P_{\bar{\kappa}}^{(b)}$, at least if we forget about the algebraic group structure. Assume that this cover is given by

$$
\bar{z}^{p}-\bar{z}+\left(\bar{\alpha}_{0} \pi_{k}^{-b-1} \delta_{0}+\bar{\alpha}_{1} \pi_{k}^{-b} \delta_{1}+\cdots+\bar{\alpha}_{m} \pi_{k}^{-b} \delta_{m}\right)=0
$$

for some $\bar{\alpha}_{J^{+}} \in \bar{\kappa}$, where the coordinates of $P_{\bar{\kappa}}^{(b)}$ are given by $\pi_{k}^{-b-1} \delta_{0}$ and $\pi_{k}^{-b} \delta_{J}$. These elements $\bar{\alpha}_{0}, \ldots, \bar{\alpha}_{m}$ are determined up to multiplication by $i \in \mathbb{F}_{p}^{\times}$, in accordance with the choice of $\bar{z}$ up to multiplication by the same $i \in \mathbb{F}_{p}^{\times}$.

Let $k^{\prime} / k$ be a finite separable extension of ramification degree $e>1$, such that $Q_{\mathcal{O}_{k^{\prime}}}^{(e b)}$ is a stable model. By possibly enlarging $k^{\prime}$, we may assume that $\bar{\alpha}_{J^{+}} \in \kappa_{k^{\prime}}$ and that $Q_{\kappa_{k^{\prime}}}^{(e b)}$ is the disjoint union of $[l: k] / p$ copies of the aforementioned ArtinScheier cover of $P_{\kappa_{k^{\prime}}}^{(e b)}$.

Lemma 3.1.14. The space $Q_{\mathscr{O}_{k^{\prime}}}^{(e b)}$ is the disjoint union of $[l: k] / p$ copies of the same space $R_{\mathscr{O}_{k^{\prime}}}^{(e b)}$. Let $\widehat{R}_{\mathscr{O}_{k^{\prime}}}^{(e b)}$ denote the completion of $R_{\bigcirc_{k^{\prime}}}^{(e b)}$ along its special fiber and let $\widehat{R}_{k^{\prime}}^{(e b)}$ denote the generic fiber, viewed as a rigid analytic space. Then $\widehat{Q}_{k^{\prime}}^{(e b-1)}$ is the disjoint union of $[l: k] / p$ copies of a same space $\widehat{R}_{k^{\prime}}^{(e b-1)}$, which is the normal closure of $\widehat{P}_{k^{\prime}}^{(e b-1)}$ in $\widehat{R}_{k^{\prime}}^{(e b)}$ and is finite and étale over $\widehat{P}_{k^{\prime}}^{(e b-1)}$.

Proof. There is a $G_{l / k}$-equivariant one-to-one correspondence between the connected components of $Q_{\kappa_{k^{\prime}}}^{(e b)}$ and the connected components of $Q_{\mathscr{O}_{k^{\prime}}}^{(e b)}$.

Since the second highest $\log$-break $b_{\log }(l / k ; 2)$ is strictly less than $b_{\log }(l / k)-1$, by [Abbes and Saito 2002, Remark 3.13], the number of connected components of $\widehat{Q}_{k^{\prime}}^{(e b-1)}$ is $[l: k] / p$. Note that each connected component of $\widehat{Q}_{k^{\prime}}^{(e b-1)}$, which is 
automatically finite and étale over $\widehat{P}_{k^{\prime}}^{(e b-1)}$, can be also characterized as the normal closure of $\widehat{P}_{k^{\prime}}^{(e b-1)}$ in $\widehat{R}_{k^{\prime}}^{(e b)}$; this normal closure is the space $\widehat{R}_{k^{\prime}}^{(e b-1)}$ we sought.

Proposition 3.1.15. Let $\alpha_{J^{+}} \subset \mathbb{O}_{k^{\prime}}$ lift $\bar{\alpha}_{J^{+}} \subset \kappa_{k^{\prime}}$. We can choose a lift $z$ of $\bar{z}$ to $\widehat{R}_{\bigcirc_{k^{\prime}}}^{(e b)}$ such that its minimal polynomial over $\widehat{P}_{\mathscr{O}_{k^{\prime}}}^{(e b)}=\operatorname{Spf}_{k^{\prime}}\left\langle\pi_{k^{\prime}}^{-e b-e} \delta_{0}, \pi_{k^{\prime}}^{-e b} \delta_{J}\right\rangle$ is

$$
z^{p}-z+\left(\alpha_{0} \pi_{k^{\prime}}^{-e b-e} \delta_{0}+\alpha_{1} \pi_{k^{\prime}}^{-e b} \delta_{1}+\cdots+\alpha_{m} \pi_{k^{\prime}}^{-e b} \delta_{m}\right)=0 .
$$

Then the element $z$ generates $\widehat{R}_{\mathrm{O}_{k^{\prime}}}^{(e b)}$ over $\widehat{P}_{\mathrm{O}_{k^{\prime}}}^{(e b)}$. Moreover, the element $z$ extends to a section over $\widehat{R}_{k^{\prime}}^{(e b-1)}$ and it generates $\widehat{R}_{k^{\prime}}^{(e b-1)}$ over $\widehat{P}_{k^{\prime}}^{(e b-1)}$.

Proof. We first pick any lift $z^{\prime}$ of $\bar{z}$ to $\widehat{R}_{\mathrm{O}_{k^{\prime}}}^{(e b)}$; it must satisfy an equation of the form $z^{\prime p}+a_{1} z^{\prime p-1}+\cdots+a_{p}=0$, where $a_{1}, \ldots, a_{p} \in \mathbb{O}_{k^{\prime}}\left\langle\pi_{k^{\prime}}^{-e b-e} \delta_{0}, \pi_{k^{\prime}}^{-e b} \delta_{J}\right\rangle$ and the reduction of this equation is exactly (3.1.13). For the given $\alpha_{J^{+}} \subset \mathrm{O}_{k^{\prime}}$, we have

$$
\epsilon=z^{\prime p}-z^{\prime}+\left(\alpha_{0} \pi_{k^{\prime}}^{-e b-e} \delta_{0}+\alpha_{1} \pi_{k^{\prime}}^{-e b} \delta_{1}+\cdots+\alpha_{m} \pi_{k^{\prime}}^{-e b} \delta_{m}\right) \in \pi_{k^{\prime}} \widehat{C}_{\widehat{R}_{\mathrm{O}_{k^{\prime}}}^{(e b)}} .
$$

Now, $z=z^{\prime}+\epsilon+\epsilon^{p}+\epsilon^{p^{2}}+\cdots$ converges and satisfies (3.1.16).

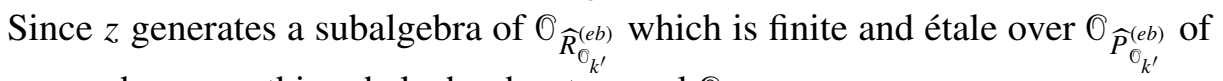
the same degree $p$, this subalgebra has to equal $0_{\widehat{R}_{\mathrm{K}^{\prime}}^{(e b)}}$.

For the similar statement for $e b-1$ in place of $e b$, we argue as follows. Since $\widehat{R}_{k^{\prime}}^{(e b-1)}$ is the normal closure of $\widehat{P}_{k^{\prime}}^{(e b-1)}$ in $\widehat{R}_{k^{\prime}}^{(e b)}$ by Lemma 3.1 .14 , the element $z$ extends to a section over $\widehat{R}_{k^{\prime}}^{(e b-1)}$ with the same minimal polynomial (3.1.16). Again, since $z$ generates a subalgebra of $\widetilde{O}_{\widehat{R}_{k^{\prime}}^{(e b-1)}}$ which is finite and étale over $\widetilde{O}_{\widehat{P}_{k^{\prime}}^{(e b-1)}}$ of same degree, it has to generate the whole ring. This finishes the proof.

3.2. Lifting rigid spaces. The definition of the refined Swan conductor homomorphism using differential modules makes use of spaces and modules over the field $K$. Following the idea of [Xiao 2010], we formally lift the picture of the previous subsection from $k$ to some annulus $A_{K}^{1}[\eta, 1)$. This construction is a local version of Berthelot's definition [1996] of rigid cohomology.

Construction 3.2.1. Replacing $X$ by an open Zariski neighborhood of $\xi$ if necessary, there exists a finite morphism $\boldsymbol{f}: \boldsymbol{Y} \rightarrow \boldsymbol{X}$ between two affine smooth formal schemes of topologically finite type over $O_{K_{0}}$, such that $f$ reduces to $f$ modulo $p$ and such that the induced map $\boldsymbol{Y} \backslash f^{-1}(D) \rightarrow \boldsymbol{X} \backslash D$ is finite étale with Galois group $G_{l / k}$. In particular, the special fibers of $\boldsymbol{X}$ and $\boldsymbol{Y}$ are $X$ and $Y$, respectively.

Let $\mathbf{\Delta}_{X}: X \rightarrow \boldsymbol{X} \times{ }_{\text {Spf }{ }_{K_{0}}} \boldsymbol{X}$ be the diagonal embedding, and put $\boldsymbol{\Delta}_{Y}=($ id, $\boldsymbol{f})$ : $\boldsymbol{Y} \rightarrow \boldsymbol{Y} \times{ }_{\mathrm{Spf}^{\circ}{ }_{K_{0}}} \boldsymbol{X}$. Let $p_{1}$ and $p_{2}$ denote the projections from $\boldsymbol{X} \times{ }_{\operatorname{Spf}{ }_{K_{0}}} \boldsymbol{X}$ to the first and the second factors, respectively.

Let $\boldsymbol{X}^{\wedge}$ denote the completion of $\boldsymbol{X} \times{ }_{\operatorname{Spf} \mathscr{O}_{K_{0}}} \boldsymbol{X}$ along the diagonal embedding $\boldsymbol{\Delta}_{X}$; it can be identified with the completion of the cotangent bundle of $\boldsymbol{X}$ along its 
zero section. Set $\boldsymbol{Y}^{\wedge}=\boldsymbol{X}^{\wedge} \otimes_{p_{1}, \boldsymbol{X}} \boldsymbol{Y}$; it is the same as the completion of $\boldsymbol{Y} \times{ }_{\mathrm{Spf}^{\circ} \mathscr{O}_{K_{0}}} \boldsymbol{X}$ along the embedding $\boldsymbol{\Delta}_{Y}$.

For $\eta \in(0,1)$, we set $\mathscr{R}_{K, \eta}^{\text {int }}$ to be the subring of $\mathscr{R}_{K}^{\eta}$ consisting of elements having 1 -Gauss norm $\leq 1$; it is complete with respect to the $\eta^{\prime}$-Gauss norm for $\eta^{\prime} \in[\eta, 1]$. On one hand, this ring does not give rise to a formal scheme; on the other hand, it is good to keep the geometric intuition. Hence we introduce the geometric incarnation $\operatorname{Sp} \Re_{K, \eta}^{\text {int }}$, which is just a symbol. Any morphism between geometric incarnations should be thought of as ring homomorphisms; in particular, the fiber product is simply the (completed) tensor product. We also point out that we will only consider affine schemes and there is no question of gluing.

We may compare the following commutative diagram with (3.1.5).

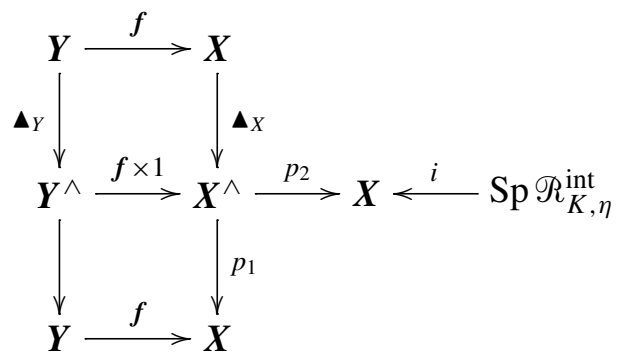

where $i: \mathrm{Sp}_{K, \eta}^{\mathrm{int}} \rightarrow \boldsymbol{X}$ is the geometric incarnation of the natural homomorphism $\mathcal{O}_{\boldsymbol{X}, \xi}^{\wedge} \rightarrow \mathscr{R}_{K, \eta}^{\mathrm{int}}$, for some $\eta \in(0,1) \cap p^{\mathbb{Q}}$. We have

$$
\operatorname{Sp} \mathscr{R}_{K, \eta}^{\mathrm{int}} \times{ }_{\boldsymbol{X}} \boldsymbol{Y}=\operatorname{Sp} \mathscr{R}_{L, \eta^{1 / e_{l / k}}}^{\mathrm{int}}
$$

for $\eta$ sufficiently close to $1^{-}$. Put

$$
\boldsymbol{P}_{\eta}=\boldsymbol{X}^{\wedge} \times_{p_{2}, \boldsymbol{X}, i} \operatorname{Sp} \mathscr{R}_{K, \eta}^{\mathrm{int}} \text { and } \boldsymbol{Q}_{\eta}=\boldsymbol{Y}^{\wedge} \times_{p_{2} \circ(\boldsymbol{f} \times 1), \boldsymbol{X}, i} \operatorname{Sp} \mathscr{R}_{K, \eta}^{\mathrm{int}} .
$$

Again, both $\boldsymbol{P}_{\eta}$ and $\boldsymbol{Q}_{\eta}$ should be thought of as geometric incarnations of $\mathcal{O}_{\boldsymbol{P}}$ and ${ }^{0} \boldsymbol{Q}_{\eta}$, the completed tensor products of corresponding rings of functions. We then have the following Cartesian diagram

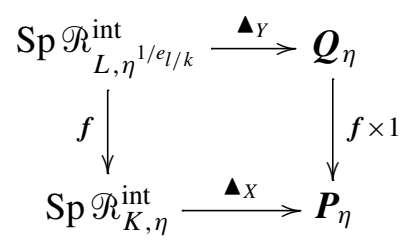

Lemma 3.2.4. The morphism $p_{1}: \boldsymbol{P}_{\eta} \rightarrow \operatorname{Sp}\left(\mathscr{R}_{K, \eta}^{\mathrm{int}}\right)$ is given by the continuous homomorphism $\psi: \mathscr{R}_{K, \eta}^{\mathrm{int}} \rightarrow \mathscr{R}_{K, \eta}^{\mathrm{int}} \llbracket \delta_{0} / T, \delta_{1}, \ldots, \delta_{m} \rrbracket$ such that $\psi(T)=T+\delta_{0}$, 
$\psi\left(B_{j}\right)=B_{j}+\delta_{j}$ for $j \in J$. More precisely, for $x \in \mathscr{R}_{K, \eta}^{\mathrm{int}}$, we have

$$
\psi(x)=\sum_{e_{J^{+}}=0}^{+\infty} \frac{\partial_{J^{+}}^{e_{J^{+}}}(x)}{\left(e_{J^{+}}\right) !} \delta_{J^{+}}^{e_{J^{+}}} .
$$

Proof. The first statement follows from the description of $\boldsymbol{X}^{\wedge}$ above and the second statement follows by the uniqueness of such a homomorphism.

Construction 3.2.5. Let $k^{\prime} / k$ be a finite separable extension of naïve ramification degree $e$. Since $\mathscr{R}_{K}^{\text {int }}$ is Henselian, there exists $\mathscr{R}_{K^{\prime}}^{\text {int }}$ corresponding to the extension $k^{\prime} / k$, where $K^{\prime}$ is the fraction field of a Cohen ring of $\kappa_{k^{\prime}}$. For $\eta$ sufficiently close to $1^{-}$, the extension $\mathscr{R}_{K^{\prime}}^{\text {int }}$ of $\mathscr{R}_{K}^{\text {int }}$ descends to a finite étale algebra $\mathscr{R}_{K^{\prime}, \eta^{1 / e}}^{\text {int }}$ over $\mathscr{R}_{K, \eta}^{\text {int }}$ for some $\eta$ sufficiently close to 1 . Fix such an $\eta$. Let $T^{\prime}$ denote the coordinate of $\mathscr{\Re}_{K^{\prime}, \eta^{1 / e}}^{\text {int }}$.

Let $r \in \mathbb{N}$ (be a proxy of $e b$ or $e b-1$ ). Let

$$
\boldsymbol{P}_{K^{\prime}, \eta}^{(r)}=\operatorname{Sp}\left(\mathscr{R}_{K^{\prime}, \eta^{1 / e}}^{\mathrm{int}}\left\langle T^{\prime-r-e} \delta_{0}, T^{\prime-r} \delta_{J}\right\rangle\right)
$$

be the geometric incarnation of a closed-disc bundle (with changing radii) over $\operatorname{Sp} \Re_{K^{\prime}, \eta^{1 / e}}^{\text {int }}$; it may be viewed as a subspace of $\boldsymbol{P}_{\eta}$ (in the sense of geometric incarnation). Let $\boldsymbol{Q}_{K^{\prime}, \eta}^{(r)}$ be the preimage (in the sense of geometric incarnation) of $\boldsymbol{P}_{K^{\prime}, \eta}^{(r)}$ under the morphism $\boldsymbol{Q}_{\eta} \rightarrow \boldsymbol{P}_{\eta}$.

Proposition 3.2.6. Let $\rho$ be a $p$-adic representation of $G_{l / k}$. Let

$$
\mathscr{F}_{\rho}=\left((\boldsymbol{f} \times 1)_{*} \mathcal{O} \boldsymbol{Q}_{\eta} \otimes V_{\rho}\right)^{G_{l / k}}
$$

be the differential module over $\boldsymbol{P}_{\eta}$ and for $r \in \mathbb{N}$, let

$$
\mathscr{F}_{\rho, K^{\prime}}^{(r)}=\left((f \times 1)_{*} \mathcal{O}_{\boldsymbol{Q}_{K^{\prime}, \eta}^{(r)}} \otimes V_{\rho}\right)^{G_{l / k}}
$$

be the corresponding differential module over $\boldsymbol{P}_{K^{\prime}, \eta^{\prime}}^{(r)}$. Then $\mathscr{F}_{\rho}$ and $\mathscr{F}_{\rho, K^{\prime}}^{(r)}$, are the pullbacks of $\mathscr{E}_{\rho}$ along $p_{1}: \boldsymbol{P}_{\eta} \rightarrow \operatorname{Sp} \mathscr{R}_{K, \eta}^{\text {int }}$ and $p_{1}: \boldsymbol{P}_{K^{\prime}, \eta}^{(r)} \rightarrow \operatorname{Sp} \mathscr{R}_{K, \eta}^{\mathrm{int}}$, respectively. Proof. This follows from the following $G_{l / k}$-equivariant Cartesian diagram of geometric incarnated morphisms.

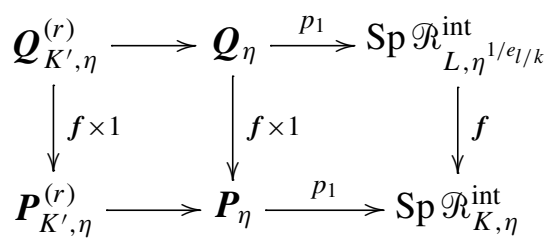

Corollary 3.2.7. For $a \in \mathbb{Q}_{<b}$ and $\eta \in(0,1) \cap p^{\mathbb{Q}}$, let $F_{\eta, a}$ denote the completion of $K\left(T, \delta_{J^{+}}\right)$with respect to the $\left(\eta, \eta^{a+1}, \eta^{a}, \ldots, \eta^{a}\right)$-Gauss norm and let 
$F_{\eta, a}^{\prime}=F_{\eta, a} \otimes_{\mathscr{R}_{K, \eta}}^{\mathrm{int}} \Re_{K^{\prime}, \eta^{1 / e}}^{\mathrm{int}}$. Assume that $\rho$ has pure log-break $b$ and pure refined Swan conductor

$$
\vartheta=\pi_{k}^{-b}\left(\bar{\alpha}_{0} \frac{d \pi_{k}}{\pi_{k}}+\bar{\alpha}_{1} \frac{d \bar{b}_{1}}{\bar{b}_{1}}+\cdots+\bar{\alpha}_{m} \frac{d \bar{b}_{m}}{\bar{b}_{m}}\right),
$$

where $\bar{\alpha}_{J^{+}} \in \bar{\kappa}$. If $r<e a<e b$ and $\eta$ is sufficiently close to $1^{-}$, then $\mathscr{F}_{\rho} \otimes F_{\eta, a}^{\prime}=$

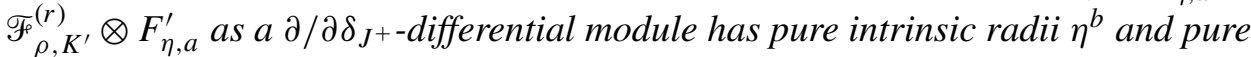
refined intrinsic radii

$$
T^{-b}\left(\bar{\alpha}_{0} \frac{d \delta_{0}}{T}+\bar{\alpha}_{1} \frac{d \delta_{1}}{B_{1}}+\cdots+\bar{\alpha}_{m} \frac{d \delta_{m}}{B_{m}}\right) .
$$

Proof. By Lemma 3.2.4 and Proposition 3.2.6, $\mathscr{F}_{\rho, K^{\prime}}^{(r)}$ is the pullback of $\mathscr{E}_{\rho}^{\mathscr{E}}$ along the multidimensional analog of the generic point homomorphism as in Corollary 1.4.21. However, the calculation of the refined $\partial_{j}$-radii can be computed independently for each of the $\partial_{j}$. Hence the statement follows from Corollary 1.4.21.

Before proceeding, we briefly recall the lifting construction in [Xiao 2010, Section 1], which lifts a rigid analytic space over $\kappa_{k^{\prime}}$ to a rigid analytic space over $A_{K^{\prime}}^{1}\left[\eta^{1 / e}, 1\right)$ for $\eta \in p^{\mathbb{Q}} \cap(0,1)$ sufficiently close to $1^{-}$.

Construction 3.2.8. Let $Z$ be a rigid analytic space over $k^{\prime}$ with ring of analytic functions $A_{k^{\prime}}=k^{\prime}\left\langle u_{1}, \ldots, u_{s}\right\rangle / I_{k^{\prime}}$. Let $I_{K^{\prime}} \subset \mathcal{O}_{K^{\prime}}\left\langle u_{1}, \ldots, u_{s}\right\rangle\left(\left(T^{\prime}\right)\right)$ be an ideal such that $\mathscr{O}_{K^{\prime}}\left\langle u_{1}, \ldots, u_{s}\right\rangle\left(\left(T^{\prime}\right)\right) / I_{K^{\prime}}$ is flat over $\mathscr{O}_{K^{\prime}}$ and $I_{K^{\prime}} \otimes_{\mathscr{O}_{K^{\prime}}} k^{\prime}=I_{k^{\prime}}$. We call $\boldsymbol{X}_{\eta}=\operatorname{Spf}\left(\mathscr{R}_{K^{\prime}, \eta}^{\mathrm{int}}\left\langle u_{1}, \ldots, u_{s}\right\rangle / I_{K^{\prime}}\right)$ a lifting space of $X$.

Proposition 3.2.9. Fix $r \in \mathbb{N}$.

(a) The space $\boldsymbol{Q}_{K^{\prime}, \eta}^{(r)}$ is a lifting space of $\widehat{Q}_{k^{\prime}}^{(r)}$.

(b) Suppose $Q_{\mathbb{O}_{k^{\prime}}}^{(r)}$ is a stable model and $r=e b$ or eb -1 . Then for $\eta$ sufficiently close to $1^{-}, \boldsymbol{Q}_{K^{\prime}, \eta}^{(r)}$ has $[l: k] / p$ connected components, each of which is isomorphic to a formal scheme $\boldsymbol{R}_{K^{\prime}, \eta}^{(r)}$ finite and étale over $\boldsymbol{P}_{K^{\prime}, \eta}^{(r)}$ of degree $p$.

(c) Fix a Dwork pi $\pi=(-p)^{1 /(p-1)}$ and fix $\alpha_{J^{+}} \subset \mathscr{R}_{K^{\prime}(\pi), \eta^{1 / e}}^{\mathrm{int}}$ lifts of $\alpha_{J^{+}}$. By making $\eta$ closer to $1^{-}$if needed, we may choose a lift $\boldsymbol{z}$ of $z$ to $\boldsymbol{R}_{K^{\prime}(\pi), \eta}^{(r)}$ whose minimal polynomial over $\boldsymbol{P}_{K^{\prime}, \eta}^{(r)}$ is of the form

$$
\frac{1}{p \pi}\left((1+\pi z)^{p}-1-p \pi\left(\boldsymbol{\alpha}_{0} T^{\prime-e b-e} \delta_{0}+\boldsymbol{\alpha}_{1} T^{\prime-e b}+\cdots+\boldsymbol{\alpha}_{m} T^{\prime-e b}\right)\right)=0 .
$$

Proof. The first statement follows from the construction. The second statement follows from [Xiao 2010, Proposition 1.2.11]; the fact that all the connected components are isomorphic to the same $\boldsymbol{R}_{K^{\prime}(\boldsymbol{\pi}), \eta}^{(r)}$ is a corollary of (c), proved below.

For (c), pick a lift $z_{1}$ of $z$ to $\boldsymbol{R}_{K^{\prime}(\boldsymbol{\pi}), \eta}^{(r)}$ whose minimal polynomial reduces to (3.1.16) modulo $\pi$. (Note that $K$ is absolutely unramified.) We define the following 
substitution process. Assume that we have defined $z_{i}$. We set

$$
\lambda_{i}=\frac{1}{p \pi}\left(\left(1+\pi z_{i}\right)^{p}-1-p \boldsymbol{\pi}\left(\boldsymbol{\alpha}_{0} T^{\prime-e b-e} \delta_{0}+\boldsymbol{\alpha}_{1} T^{-e b}+\cdots+\boldsymbol{\alpha}_{m} T^{-e b}\right)\right) .
$$

and set $z_{i+1}=z_{i}-\lambda_{i}$. Hence we have

$$
\begin{aligned}
\boldsymbol{\lambda}_{i+1} & =\frac{1}{p \boldsymbol{\pi}}\left(\left(1+\boldsymbol{\pi} z_{i}-\boldsymbol{\pi} \boldsymbol{\lambda}_{i}\right)^{p}-\left(1+\boldsymbol{\pi} z_{i}\right)^{p}+p \boldsymbol{\pi} \boldsymbol{\lambda}_{i}\right) \\
& =\left(1-\left(1+\boldsymbol{\pi} z_{i}\right)^{p-1}\right) \boldsymbol{\lambda}_{i}+\sum_{n=2}^{p-1} \frac{1}{p \boldsymbol{\pi}}\left(\begin{array}{l}
p \\
n
\end{array}\right)\left(1+\boldsymbol{\pi} z_{i}\right)^{p-n}\left(-\boldsymbol{\pi} \boldsymbol{\lambda}_{i}\right)^{n}+(-1)^{p-1} \boldsymbol{\lambda}_{i}^{p} .
\end{aligned}
$$

Since $\left|\lambda_{1}\right|_{1} \leq p^{-1 /(p-1)}$, by continuity, $\left|\lambda_{1}\right|_{\eta}<1$ for $\eta \in\left[\eta_{0}, 1\right]$ for some $\eta_{0}$ sufficiently close to $1^{-}$. Thus,

$$
\left|\lambda_{i+1}\right|_{\eta} \leq \max \left\{p^{-1 /(p-1)}\left|\lambda_{i}\right|_{\eta},\left|\lambda_{i}\right|_{\eta}^{p}\right\} \quad \text { for } \eta \in\left[\eta_{0}, 1\right) .
$$

As a consequence, this substitution process converges with respect to all $\eta$-Gauss norms for $\eta \in\left[\eta_{0}, 1\right]$. The limit $z=\lim _{i \rightarrow+\infty} z_{i}$ satisfies (3.2.10). By the same argument as in Proposition 3.1.15, the limit $z$ generates $\boldsymbol{R}_{K^{\prime}(\boldsymbol{\pi}), \eta}^{(r)}$ over $\boldsymbol{P}_{K^{\prime}(\boldsymbol{\pi}), \eta}^{(r)}$ when $\eta$ is sufficiently close to $1^{-}$.

3.3. Dwork isocrystals. In this subsection, we single out a calculation of refined radii for the differential modules coming from a higher dimensional Artin-Scheier cover. This is the heart of the comparison Theorem 3.4.1. We will state it in a slightly general form because it has its own interest in the study of differential modules.

Hypothesis 3.3.1. In this subsection, let $K$ be a complete discrete valuation field of characteristic zero, containing $\pi$. Let $\kappa$ denote its residue field, which has characteristic $p>0$.

Situation 3.3.2. Let $\boldsymbol{P}$ denote the formal scheme $\operatorname{Spf} \mathscr{R}_{K, \eta}^{\mathrm{int}}\left\langle\delta_{0}, \ldots, \delta_{m}\right\rangle$, and let $T$ be the coordinate of $R_{K, \eta}^{\text {int }}$. Let $\boldsymbol{R}$ be a finite extension of $\boldsymbol{P}$ generated by $\boldsymbol{z}$ satisfying the relation

$$
(1+\pi \boldsymbol{z})^{p}=1+p \pi T^{-r}\left(\boldsymbol{\alpha}_{0} \delta_{0}+\cdots+\boldsymbol{\alpha}_{m} \delta_{m}\right),
$$

where $r \in \mathbb{N}$ and $\boldsymbol{\alpha}_{j} \in \mathscr{R}_{K, \eta}^{\text {int }}$ for $j=1, \ldots, m$. Let $\alpha_{j} \in \kappa$ be the reduction of $\boldsymbol{\alpha}_{j}$ for any $j$. We assume that not all $\alpha_{j}$ are zero. Let $f: \boldsymbol{R} \rightarrow \boldsymbol{P}$ be the natural morphism, which is finite and étale.

Construction 3.3.3. We reproduce a multidimensional version of the construction in [Kedlaya 2005, Lemma 5.4.7]. The pushforward $f_{*} \mathcal{O}_{\boldsymbol{Q}}$ decomposes as the direct sum of $p$ differential modules of rank 1 , with respect to $\partial_{j}=\partial / \partial \delta_{j}$ for $j=0, \ldots, m$.

Let $\mathscr{E}_{i}$ be the differential module given by $(1+\pi z)^{i}$ for $i=1, \ldots, p-1$. (The trivial submodule of $f_{*} \mathrm{O} Q$ is not of interest to us.) 
Notation 3.3.4. For $\eta \in(0,1)$, let $\boldsymbol{F}_{\eta}$ be the completion of $K\left(T, \delta_{0}, \ldots, \delta_{m}\right)$ with respect to the $(\eta, 1, \ldots, 1)$-Gauss norm.

Proposition 3.3.5. For $\eta$ sufficiently close to $1^{-}$, the intrinsic radius $\operatorname{IR}\left(\mathscr{E}_{i} \otimes \boldsymbol{F}_{\eta}\right)$ is equal to $\eta^{r}$ and the refined intrinsic radius of $\mathscr{E}_{i}$ for $i=1, \ldots, p-1$ is given by

$$
g \Theta\left(\mathscr{E}_{i} \otimes \boldsymbol{F}_{\eta}\right)=\left\{i \pi T^{-r}\left(\alpha_{0} d \delta_{0}+\cdots+\alpha_{m} d \delta_{m}\right)\right\} .
$$

Proof. Since

$$
p \frac{d(1+\boldsymbol{\pi} z)^{i}}{(1+\boldsymbol{\pi} \boldsymbol{z})^{i}}=i \frac{d\left(1+p \boldsymbol{\pi} T^{-r}\left(\boldsymbol{\alpha}_{0} \delta_{0}+\cdots+\boldsymbol{\alpha}_{m} \delta_{m}\right)\right)}{1+p \boldsymbol{\pi} T^{-r}\left(\boldsymbol{\alpha}_{0} \delta_{0}+\cdots+\boldsymbol{\alpha}_{m} \delta_{m}\right)},
$$

$\mathscr{E}_{i}$ is isomorphic to a differential module given by

$$
\nabla \boldsymbol{v}=i \boldsymbol{\pi} T^{-r}\left(1+p \boldsymbol{\pi} T^{-r}\left(\boldsymbol{\alpha}_{0} \delta_{0}+\cdots+\boldsymbol{\alpha}_{m} \delta_{m}\right)\right)^{-1} \boldsymbol{v} \otimes\left(\boldsymbol{\alpha}_{0} d \delta_{0}+\cdots+\boldsymbol{\alpha}_{m} d \delta_{m}\right) .
$$

Fix $j=0, \ldots, m$. Using the proof of [Kedlaya 2005, Lemma 5.4.7], when $\eta$ is sufficiently close to $1^{-}$(e.g., $\eta>p^{-1 / r}$ ), viewed as a $\partial_{j}$-differential module, this is the same as

$$
\partial_{j} \boldsymbol{w}_{j}=i \pi \boldsymbol{\alpha}_{j} T^{-r} \boldsymbol{w}_{j}
$$

where $\boldsymbol{w}_{j}$ is a section of $\mathscr{E}_{i}$, dependent on $j$. Hence $\partial_{j}^{n}\left(\boldsymbol{w}_{j}\right)=\left(i \boldsymbol{\pi} \boldsymbol{\alpha}_{j} T^{-r}\right)^{n} \boldsymbol{w}_{j}$, and the proposition follows immediately.

3.4. Comparison. In this subsection, we assemble the results from previous subsections to prove the following comparison theorem.

Theorem 3.4.1. Assume Hypothesis (Geom). Then for $b \in \mathbb{Q}_{>0}$, the homomorphism rsw : $\operatorname{Hom}\left(\mathrm{Fil}_{\log }^{b} G_{k} / \mathrm{Fil}_{\log }^{b+} G_{k}, \mathbb{F}_{p}\right) \rightarrow \Omega_{k}^{1}(\log ) \otimes \pi_{k}^{-b} \bar{\kappa}$ in Theorem 2.3.7 is the same as the homomorphism rsw' in Proposition 3.1.11.

Proof. Let $\tilde{k}$ be as in Proposition 2.2.14. By [Saito 2009, Lemma 1.22], rsw' for $k$ factors as

$$
\begin{aligned}
\operatorname{Hom}\left(\mathrm{Fil}_{\log }^{b} G_{k} / \mathrm{Fil}_{\log }^{b+} G_{k}, \mathbb{F}_{p}\right) \rightarrow \operatorname{Hom}\left(\mathrm{Fil}_{\log }^{e_{\tilde{k} / k} b} G_{\tilde{k}} / \operatorname{Fil}_{\log }^{\left(e_{\tilde{k} / k} b\right)+} G_{\tilde{k}}, \mathbb{F}_{p}\right) & \\
& \stackrel{\operatorname{rsw}_{\tilde{k}}^{\prime}}{\longrightarrow} \Omega_{\mathscr{\complement}_{\tilde{k}}}^{1}(\log ) \otimes_{\mathscr{C}_{\tilde{k}}} \pi_{\tilde{k}}^{-e_{\tilde{k} / k} b} \kappa_{\tilde{k} \text { alg }} .
\end{aligned}
$$

The same factorization is also valid for rsw as in (2.3.12). Hence we may choose $e_{\tilde{k} / k}$ divisible by the denominator of $b$ and reduce to the case when $b$ is an integer. We also remark that, for the same reason, we may feel free to replace $k$ by a finite tamely ramified extension.

Fix $\zeta_{p}$ a $p$-th root of unity. Let $\chi: \mathrm{Fil}_{\log }^{b} G_{k} / \mathrm{Fil}_{\log }^{b+} G_{k} \rightarrow \mathbb{F}_{p}$ be a nontrivial character and put

$$
\operatorname{rsw}^{\prime}(\chi)=\pi_{k}^{-b}\left(\bar{\alpha}_{0} \frac{d \pi_{k}}{\pi_{k}}+\bar{\alpha}_{1} d \bar{b}_{1}+\cdots+\bar{\alpha}_{m} d \bar{b}_{m}\right),
$$


where $\bar{\alpha}_{0}, \ldots, \bar{\alpha}_{m} \in \bar{\kappa}$. By identifying $1 \in \mathbb{F}_{p}$ with $\zeta_{p} \in \mathbb{Q}_{p}\left(\zeta_{p}\right)$, we get a homomorphism

$$
\mathrm{Fil}_{\log }^{b} G_{k} / \mathrm{Fil}_{\log }^{b+} G_{k} \stackrel{\chi}{\rightarrow} \mathbb{F}_{p} \rightarrow \mathbb{Q}_{p}\left(\zeta_{p}\right)^{\times} ;
$$

we still use $\chi$ to denote the composition. By the argument and the result of Theorem 2.3 .7 and by possibly replacing $k$ by a finite tamely ramified extension, we can find a $p$-adic representation $\rho$ of $G_{k}$ with finite image and pure log-break $b$ such that $\left.\rho\right|_{\mathrm{Fil}_{\log }^{b} G_{k}}$ is a direct sum of copies of $\chi$. Moreover, we may assume that $\rho$ is irreducible when restricted to any finite tamely ramified extension of $k^{\prime}$ of $k$. The representation $\rho$ factors exactly through $l / k$, a finite Galois extension. It must be true that $\mathrm{Fil}_{\log }^{b} G_{k} / G_{l} \cap \mathrm{Fil}_{\log }^{b} G_{k} \simeq \mathbb{F}_{p}$. By possibly making another tamely ramified extension of $k$, we may assume that the second highest log-break of $l / k$ is strictly less than $b-1$; thus $\mathrm{Fil}_{\log }^{b-1} G_{k} / G_{l} \cap \mathrm{Fil}_{\log }^{b-1} G_{k} \simeq \mathbb{F}_{p}$.

We shall now use the results and notation from previous subsections. By Proposition 3.2.9, $\boldsymbol{Q}_{K^{\prime}, \eta}^{(e b-1)}$ is a disjoint union of $[l: k] / p$ copies of $\boldsymbol{R}_{K^{\prime}, \eta}^{(e b-1)}$, which is finite and étale over $\boldsymbol{P}_{K^{\prime}, \eta}^{(e b-1)}$, generated by $\boldsymbol{z}$ with minimal polynomial (3.2.10). (Here, we made a choice of $z$ and $z$ in accordance with the algebraic group structure on $Q_{\bar{\kappa}}^{b}$; see the remarks after (3.1.13).) By Proposition 3.3.5, this implies that $\underset{\rho, K^{\prime}}{\mathscr{F}^{e}} \otimes F_{\eta, b-1 / 2 e}^{\prime}$ as $\eta \rightarrow 1^{-}$has pure refined intrinsic radii

$$
\pi T^{-b}\left(\bar{\alpha}_{0} \frac{d \delta_{0}}{T}+\bar{\alpha}_{1} d \delta_{1}+\cdots+\bar{\alpha}_{m} d \delta_{m}\right) .
$$

(Here we made a choice of Dwork pi $\pi$ so that $\pi \equiv \zeta_{p}-1 \bmod \left(\zeta_{p}-1\right)^{2}$ as in Remark 2.3.4.) By Corollary 3.2.7, the refined Swan conductor of $\mathscr{E}_{\rho}$ has to be $\pi_{k}^{-b}\left(\bar{\alpha}_{0} \frac{d \pi_{k}}{\pi_{k}}+\bar{\alpha}_{1} d \bar{b}_{1}+\cdots+\bar{\alpha}_{m} d \bar{b}_{m}\right)$, the same as rsw'.

Remark 3.4.2. By [Abbes and Saito 2009, Theorem 9.1.1], the two definitions of refined Swan conductors above are the same as Kato's definition in [Kato 1989], when the representation is one-dimensional. So all three definitions agree. This result is also implicitly contained in [Chiarellotto and Pulita 2009].

\section{Refined Swan conductors and variation of intrinsic radii on polyannuli}

When we have a differential module over a polyannulus or a polydisc, similar to the one-dimensional situation, we may study how the multiset of intrinsic radii of the differential module changes as we complete the module with respect to different Gauss norms. Kedlaya and the author [2010] proved that the partial sums of the log of intrinsic radii form continuous convex piecewise affine functions. The purpose of this section is to prove that the slopes at some point of such affine functions are related to the refined intrinsic radii of the differential module, completed with respect to the corresponding Gauss norm. Again, the proof proceeds in two steps, first over an annulus or a disc (Section 4.2) and then over a polyannulus or a polydisc 
(Section 4.3). The first subsection focuses on some technical results which will be used in the following two subsections.

Hypothesis 4.0.1. We assume Hypothesis 1.5.1 and keep the notation of Section 1 . We also assume that $K$ is discretely valued throughout this section. We do not insist $p>0$ in this section unless otherwise specified.

4.1. Partial decomposition for differential modules. In Section 1.5, we deliberately restricted ourselves to the situation over open annuli. In many applications, it is equally important to understand the theory of differential modules over a bounded analytic ring, for example $K\left\{\left\{\alpha / t, t \rrbracket_{0}\right.\right.$. This subsection is devoted to developing a parallel theory in this case, which is not addressed in [Kedlaya and Xiao 2010].

We fix some $\alpha \in(0,1)$ for this subsection.

Notation 4.1.1. We define $E$ to be the completion of Frac $\left(K\left\{\left\{\alpha / t, t \rrbracket_{0}\right)\right.\right.$ with respect to the 1-Gauss norm; it is isomorphic to the $p$-adic completion of $O_{K}((t))\left[\frac{1}{p}\right]$, and it contains $F_{1}$ as a subfield.

If $s \in-\log \left|K^{\times}\right|$, we can find an element $x \in K^{\times}$with $|x|=\mathrm{e}^{-s}$. This $x$ defines an isomorphism

$$
\kappa_{E}^{(s)} \stackrel{x^{-1}}{\longrightarrow} \kappa_{E} \cong \kappa_{K}((t))
$$

Hence we have a canonical valuation $\mathrm{v}_{S}(\cdot)$ on $\kappa_{E}^{(s)}$ given by the $t$-valuation; this does not depend on the choice of $x \in K^{\times}$. This valuation extends naturally to $\kappa_{E^{\text {alg }}}^{(s)}$ for $s \in \mathbb{Q} \cdot \log \left|K^{\times}\right|$.

Notation 4.1.2. Let $j \in J^{+}$. For $M$ a $\partial_{j}$-differential module over $K\left\{\left\{\alpha / t, t \rrbracket_{0}\right.\right.$ of rank $d$ and $i \in\{1, \ldots, d\}$, we put

$$
f_{i}^{(j)}(M, 0)=-\log R_{\partial_{j}}(M \otimes E ; i), \quad F_{i}^{(j)}(M, 0)=f_{1}^{(j)}(M, 0)+\cdots+f_{i}^{(j)}(M, 0) .
$$

We similarly define $f_{i}(M, 0)$ and $F_{i}(M, 0)$ if $M$ is a $\partial_{J^{+-}}$differential module over $K\left\{\left\{\alpha / t, t \rrbracket_{0}\right.\right.$.

Proposition 4.1.3. Fix $j \in J^{+}$. Let $M$ be a $\partial_{j^{-}}$(resp. $\partial_{J^{+-}}$) differential module of rank $d$ over $K\left\{\left\{\alpha / t, t \rrbracket_{0}\right.\right.$.

(a) The functions $f_{i}^{(j)}(M, r)$ and $F_{i}^{(j)}(M, r)$ are continuous, and are affine if $f_{i}^{(j)}(M, 0)>-\log \left|u_{j}\right|$; the functions $f_{i}(M, r)$ and $F_{i}(M, r)$ are affine.

(b) Suppose for some $i \in\{1, \ldots, d-1\}$, the function $F_{i}^{(j)}(M, r)$ (resp. $F_{i}(M, r)$ is affine), and $f_{i}^{(j)}(M, r)>f_{i+1}^{(j)}(M, r)$ (resp. $\left.f_{i}(M, r)>f_{i+1}(M, r)\right)$ for $r \in[0,-\log \alpha)$. Then $M$ admits a unique direct sum decomposition $M_{0} \oplus M_{1}$ over $K\left\{\left\{\alpha / t, t \rrbracket_{0}\right.\right.$ such that 
(i) for any $\eta \in(0,-\log \alpha)$, the multisets of subsidiary $\partial_{j}$-radii (resp. intrinsic radii) of $M_{0} \otimes F_{\eta}$ exactly consist of the $i$ smallest elements of the multisets of subsidiary $\partial_{j}$-radii (resp. intrinsic radii) of $M \otimes F_{\eta}$, and

(ii) the multisets of subsidiary $\partial_{j}$-radii (resp. intrinsic radii) of $M_{0} \otimes E$ exactly consist of the $i$ smallest elements of the multisets of subsidiary $\partial_{j}$-radii (resp. intrinsic radii) of $M \otimes E$.

Proof. The statement (a) for $\partial_{j}$-radii follows from the exact same argument as [Kedlaya and Xiao 2010, Theorem 2.2.6(a)], which follows immediately from the corresponding properties of the associated twisted polynomial. We now explain how we deduce (a) for intrinsic radii. Firstly, by parts (a), (b) and (d) of Theorem 1.5.6, $d ! \cdot F_{i}(M, r)$ is convex and piecewise affine of integer slopes for $r \in(0,-\log \alpha)$. We need only to check continuity at $r=0$, which follows from exactly the same argument as in Step 1 of the proof of [ibid., Theorem 2.3.9].

The statement (b) is proved in [ibid., Theorems 2.3.9, 2.5.5, and Remarks 2.3.11, 2.5.7].

Note that the statement (b) of the above proposition excludes the case when $f_{i}^{(j)}(M, r)>f_{i+1}^{(j)}(M, r)$ for $r \in(0,-\log \alpha)$ and $f_{i}^{(j)}(M, 0)=f_{i+1}^{(j)}(M, 0)$, and the similar case with the superscript $(j)$ removed. The rest of this subsection is devoted to extending the conclusion of (b) to this case.

Notation 4.1.4. Set $\mathscr{R}=\bigcap_{\alpha \in(0,1)} K\{\{\alpha / t, t\}\}$ and $\mathscr{R}^{\mathrm{bd}}=\bigcap_{\alpha \in(0,1)} K\left\{\left\{\alpha / t, t \rrbracket_{0}\right.\right.$, where the latter can be identified with the subring of the former consisting of elements with finite 1-Gauss norm.

Hypothesis 4.1.5. We assume that $\left|u_{j}\right|=1$ for $j \in J$.

This hypothesis is just to make our presentation simpler. We can always reduce to this case by replacing $K$ by the completion of $K\left(x_{1}, \ldots, x_{m}\right)$ with respect to the $\left(\left|u_{1}\right|, \ldots,\left|u_{m}\right|\right)$-Gauss norm and by replacing $u_{j}$ by $u_{j} / x_{j}$, where $\partial_{j}\left(x_{j^{\prime}}\right)=0$ for $j, j^{\prime} \in J$. Note that $K$ is still discretely valued.

Lemma 4.1.6. The ring $\mathscr{R}^{\mathrm{bd}}$ is a field. A sequence $\left(f_{n}\right)_{n \in \mathbb{N}} \subset K\left\{\left\{\alpha / t, t \rrbracket_{0}\right.\right.$ is convergent if it is convergent for the $r$-Gauss norm for all $r \in(\alpha, 1)$ and is bounded for the 1-Gauss norm.

Proof. The first statement is well-known; see [Kedlaya 2005, Lemma 3.5.2]. We remark that this would be false if $K$ were not discretely valued. To see the second statement, we observe that $\left(f_{n}\right)_{n \in \mathbb{N}}$ converges in $K\{\{\alpha / t, t\}\}$. The limit has bounded coefficients and hence lies in $K\left\{\left\{\alpha / t, t \rrbracket_{0}\right.\right.$.

Lemma 4.1.7. Fix $j \in J^{+}$. Let $\mathscr{R}^{\mathrm{bd}}\{T\}$ be the ring of twisted polynomials as in Definition 1.2.1, where $T$ stands for $\partial_{j}$ if $j \in J$ and for $d / d t$ if $j=0$. Let $P=T^{d}+a_{i} T^{d-1}+\cdots+a_{d} \in \mathscr{R}^{\mathrm{bd}}\{T\}$ be a monic twisted polynomial whose Newton 
polygon has pure slope $s<1$. Let $\left\{b_{1}, \ldots, b_{r}\right\}$ be the set of $\mathrm{v}_{s}$-valuations of the reduced roots of $P$ (not counting multiplicity, with either increasing or decreasing order), when we view $P$ as a twisted polynomial in $E\{T\}$. Then $P$ admits a unique factorization $P=Q_{1} \cdots Q_{r}$ as products of monic twisted polynomials such that all the reduced roots of $Q_{i}$, when viewed as twisted polynomials in $E\{T\}$, have $\mathrm{v}_{s}$-valuations $b_{i}$.

Proof. We assume that $b_{1}, \ldots, b_{r}$ are in decreasing order. It then suffices to show that we can write $P=Q R$ as a product of two monic polynomials such that the reduced roots of $Q$ and $R$, when viewed as twisted polynomials in $E\{T\}$, have pure $v_{s}$-valuations $b_{1}$ and strictly less than $b_{1}$, respectively. We can also write it as $P=R Q$ satisfying the same condition, but with different $Q$ and $R$. By Lemma 4.1.6, the claim follows from [Kedlaya 2009, Proposition 3.2.2] because the sequences $\left\{P_{l}\right\}$ and $\left\{Q_{l}\right\}$ there are bounded under the 1-Gauss norm.

Lemma 4.1.8. Fix $j \in J$. Let $M$ be a $\partial_{j}$-differential module of rank $d$ over $K\left\{\left\{\alpha / t, t \rrbracket_{0}\right.\right.$ such that $M \otimes E$ has pure intrinsic $\partial_{j}$-radii $I R_{\partial_{j}}(M \otimes E)<\omega$. By choosing a cyclic vector of $M \otimes \mathscr{R}^{\mathrm{bd}}$, we may identify $M \otimes \mathscr{R}^{\mathrm{bd}}$ with $\mathscr{R}^{\mathrm{bd}}\{T\} / \mathscr{R}^{\mathrm{bd}}\{T\} P$, where $P$ is a twisted polynomial in $\mathscr{R}^{\mathrm{bd}}\{T\}$. Then for $\eta$ sufficiently close to $1^{-}$, the slopes of the Newton polygon of $P$ (for the $\eta$-Gauss norm) are the log of the subsidiary $\partial_{j}$-radii of $M \otimes F_{\eta}$ minus $\log \omega$.

Proof. The identification $M \otimes \mathscr{R}^{\mathrm{bd}} \simeq \mathscr{R}^{\mathrm{bd}}\{T\} / \mathscr{R}^{\mathrm{bd}}\{T\} P$ descends to

$$
M \otimes K\left\{\left\{\beta / t, t \rrbracket_{0} \simeq K\left\{\left\{\beta / t, t \rrbracket_{0}\{T\} / K\left\{\left\{\beta / t, t \rrbracket_{0}\{T\} P\right.\right.\right.\right.\right.\right.
$$

for $\beta$ sufficiently close to $1^{-}$. Note that for $\eta$ sufficiently close to $1^{-}$, all $\partial_{j}$-radii of $M \otimes F_{\eta}$ are visible. The lemma follows from Proposition 1.2.8.

The following theorem also holds without assume Hypothesis 4.1.5.

Theorem 4.1.9. Fix $j \in J^{+}$. Let $M$ be a $\partial_{j^{-}}$(resp. $\left.\partial_{J^{+-}}\right)$differential module of rank $d$ over $K\left\{\left\{\alpha / t, t \rrbracket_{0}\right.\right.$ such that $M \otimes E$ has pure intrinsic $\partial_{j}$-radii $I_{\partial_{j}}(M \otimes E)<1$ (resp. intrinsic radii $I R(M \otimes E)<1)$. Suppose that for some $i \in\{1, \ldots, d-1\}$, the function $F_{i}^{(j)}(M, r)$ (resp. $\left.F_{i}(M, r)\right)$ is affine and $f_{i}^{(j)}(M, r)>f_{i+1}^{(j)}(M, r)$ (resp. $\left.f_{i}(M, r)>f_{i+1}(M, r)\right)$ for any $r \in(0,-\log \alpha)$. Then $M$ admits a unique direct sum decomposition $M_{0} \oplus M_{1}$ of $\partial_{j}$ - (resp. $\left.\partial_{J^{+-}}\right)$differential module over $K\left\{\left\{\alpha / t, t \rrbracket_{0}\right.\right.$ such that, for any $\eta \in(0,-\log \alpha)$, the multiset of $\partial_{j}$-radii (resp. intrinsic radii) of $M_{0} \otimes F_{\eta}$ exactly consists of the smallest $i$ elements of the multiset of $\partial_{j}$-radii (resp. intrinsic radii) of $M \otimes F_{\eta}$.

Proof. We first deduce the $\partial_{j}$-differential module case. By Theorem 1.5.4(e), it suffices to obtain the decomposition over $K\left\{\left\{\beta / t, t \rrbracket_{0}\right.\right.$ for $\beta \in(\alpha, 1)$ sufficiently close to 1 and then we may apply Lemma 1.1.10 and Remark 1.1.11 to glue this decomposition with the decomposition given by Theorem 1.5.4(e). 
To start, we assume that $I R_{\partial_{j}}(M \otimes E)<\omega$. By making $\beta$ closer to 1 , we may assume that $I R_{\partial_{j}}\left(M \otimes F_{\eta}\right)<\omega$ for all $\eta \in(\beta, 1)$ as well. It is also very easy to reduce to the case when Hypothesis 4.1.5 holds. Since $\mathscr{R}^{\text {bd }}$ is a field, we can find a cyclic vector to identify $M \otimes \mathscr{R}^{\text {bd }}$ with $\mathscr{R}^{\text {bd }}\{T\} / \mathscr{R}^{\text {bd }}\{T\} P$ for a monic twisted polynomial $P$ as in Lemma 4.1.7. Applying Lemma 4.1.7 to $M \otimes \mathscr{R}^{\text {bd }}$ with the $b$ 's in decreasing order, we can find a submodule $M_{0}$ of $M$ such that the multiset of $\partial_{j}$-radii of $M_{0} \otimes F_{\eta}$ exactly consists of the smallest $i$ elements in the multiset of $\partial_{j}$-radii of $M \otimes F_{\eta}$ when $\eta$ sufficiently close to $1^{-}$. Applying Lemma 4.1.7 again with the $b$ 's increasing, we can find a quotient $M_{0}^{\prime}$ of $M$ satisfying exactly the same condition on $M_{0}$ as above. Then the kernel of $M \rightarrow M_{0}^{\prime}$ together with $M_{0}$ gives the direct sum decomposition required in the theorem.

We next assume that $p>0$ and $I R_{\partial_{j}}(M \otimes E)=p^{-1 /(p-1)}$. If $j \in J$, the $\partial_{j^{-}}$ Frobenius $\varphi^{\left(\partial_{j}\right)}: K^{\left(\partial_{j}\right)} \rightarrow K$ naturally extends to

$$
\varphi^{\left(\partial_{j}\right)}: K^{\left(\partial_{j}\right)}\left\{\left\{\alpha / t, t \rrbracket_{0} \rightarrow K\left\{\left\{\alpha / t, t \rrbracket_{0} ;\right.\right.\right.\right.
$$

if $j=0$, we have $\varphi^{\left(\partial_{0}\right)}: K\left\{\left\{\alpha^{p} / t^{p}, t^{p} \rrbracket_{0} \rightarrow K\left\{\left\{\alpha / t, t \rrbracket_{0}\right.\right.\right.\right.$. Then the desired decomposition follows from the decomposition of $\varphi_{*}^{\left(\partial_{j}\right)} M$. Note that $\varphi^{\left(\partial_{j}\right) *} \varphi_{*}^{\left(\partial_{j}\right)} M \cong M^{\oplus p}$.

If $p>0$ and $I R_{\partial_{j}}(M \otimes E)>p^{-1 /(p-1)}$, we may assume that

$$
I R_{\partial_{j}}\left(M \otimes F_{\eta}\right)>p^{\frac{-1}{p-1}}
$$

for all $\eta \in(\beta, 1)$, and the decomposition follows from that of the $\partial_{j}$-Frobenius antecedent of $M$.

Finally, we show that the $\partial_{J^{+-}}$differential module case follows from the $\partial_{j^{-}}$ differential module case. By Theorem 1.5.6(e), it suffices to find the decomposition over $K\left\{\left\{\beta / t, t \rrbracket_{0}\right.\right.$ for $\beta \in(\alpha, 1)$ sufficiently close to 1 and then, to glue the decompositions using Lemma 1.1.10 and Remark 1.1.11. By Proposition 4.1.3(a) and Theorem 1.5.4(a), there exists $\beta \in(\alpha, 1)$ such that, if $I R_{\partial_{j}}(M \otimes E ; i)<1$ for some $j$, then the function $f_{i}^{(j)}(M, r)$ for this $j$ is affine over $[0,-\log \beta)$. By the decompositions given by Proposition 4.1.3(b) and this theorem for $\partial_{j}$, the restriction of $M$ to $K\left\{\left\{\beta / t, t \rrbracket_{0}\right.\right.$ is the direct sum of $\partial_{J^{+-}}$differential modules $M_{l}$ such that, for any $j \in J^{+}$with $I R_{\partial_{j}}\left(M_{l} \otimes E\right)<1$, the $\partial_{j}$-differential module $M_{l} \otimes F_{\eta}$ has pure $\partial_{j}$-radii for any $\eta \in(\beta, 1)$. Since we already know that $M \otimes E$ has pure intrinsic radii $<1$, we may take $\beta$ sufficiently close to 1 such that each direct summand above has pure intrinsic radii equal to the $\partial_{j}$-radii for some $j$, when tensored with $F_{\eta}$ for any $\eta \in(\beta, 1)$. Hence regrouping the direct summands gives the direct sum decomposition we are looking for.

Remark 4.1.10. The condition $I R_{\partial_{j}}(M \otimes E)<1$ is crucial. As pointed out in [Kedlaya 2010, Remark 12.5.4], one may give counterexamples in the case $I R_{\partial_{j}}(M \otimes$ 
$E)=1$ using the theory of crystals. However, in the presence of a Frobenius, one may still get the decomposition.

Proposition 4.1.11. Let $M$ be a $\partial_{J^{+}}$-differential module over $K\left\{\left\{\alpha / t, t \rrbracket_{0}\right.\right.$ (resp. $\left.K \llbracket t \rrbracket_{0}\right)$ of rank $d$. We put $\hat{f}_{i}(M, 0)=-\log E R(M \otimes E ; i)$ and

$$
\hat{F}_{i}(M, 0)=\hat{f}_{1}(M, 0)+\cdots+\hat{f}_{i}(M, 0) \text { for } i=1, \ldots, d .
$$

(a) The functions $\hat{f}_{i}(M, r)$ and $\hat{F}_{i}(M, r)$ are affine at $r=0$.

(b) Suppose for some $i \in\{1, \ldots, d-1\}$, the function $\hat{F}_{i}(M, r)$ is affine and $\hat{f}_{i}(M, r)>\hat{f}_{i+1}(M, r)$ for $r \in(0,-\log \alpha)$ (resp. whenever $\left.\hat{f}_{i}(M, r)>r\right)$, and suppose that $\hat{f}_{i}(M, 0)>0$. Then $M$ admits a unique direct sum decomposition $M_{0} \oplus M_{1}$ over $K\left\{\left\{\alpha / t, t \rrbracket_{0}\right.\right.$ (resp. $\left.K \llbracket t \rrbracket_{0}\right)$ such that the multiset of extrinsic radii of $M \otimes F_{\eta}$ for any $\eta \in(0,-\log \alpha)$ (resp. for any $\eta>0$ such that $\left.\hat{f}_{i}(M, r)>r\right)$ consists of the smallest $i$ elements of the multiset of extrinsic radii of $M \otimes F_{\eta}$.

Proof. (a) follows from exactly the same argument as in Proposition 4.1.3. We now prove (b). By the extrinsic version of Theorem 1.5.6(e), it suffices to find the decomposition over $K\left\{\left\{\beta / t, t \rrbracket_{0}\right.\right.$ for $\beta \in(\alpha, 1)$ sufficiently close to 1 and then we may apply Lemma 1.1.10 and Remark 1.1.11 to glue the decompositions. By Proposition 4.1.3(b) and Theorem 4.1.9 for $\partial_{j}$-differential modules, there exists $\beta \in(\alpha, 1)$ such that when we tensor $M$ with $K\left\{\left\{\beta / t, t \rrbracket_{0}\right.\right.$, it is a direct sum of differential modules $M_{l}$ such that either for any $j \in J^{+}$with $R_{\partial_{j}}\left(M_{l} \otimes E\right)<1$, $M_{l} \otimes F_{\eta}$ has pure $\partial_{j}$-radii for all $\eta \in(\beta, 1)$, or we have $E R\left(M_{l} \otimes E\right)=1$. The proposition then follows from regrouping these direct summands.

4.2. Refined radii and the log-slopes of the radii. For a differential module over an annulus or a disc, the slopes of the functions coming from the radii of convergence are determined by the multiset of refined radii for the differential module completed for the corresponding Gauss norm. We also give a refined radii decomposition result for differential modules over bounded analytic rings.

Theorem 4.2.1. Fix $j \in J^{+}$and let $M$ be a $\partial_{j}$-differential module over $K\left\{\left\{\alpha / t, t \rrbracket_{0}\right.\right.$ of rank $d$. Assume that $f_{i}^{(j)}(M, r)$ for all $i$ are the same and are affine of slope $b$ in $r \in[0,-\log \alpha)$. Moreover, we assume that $R_{\partial_{j}}(M \otimes E)=\omega \mathrm{e}^{s}$ is strictly less than $\left|u_{j}\right|^{-1}$ if $j \in J$ and is strictly less than 1 if $j=0$. Then the $v_{s}$-valuation of any element in the multiset of refined $\partial_{j}$-radii of $M \otimes E$ is $-b$.

Proof. We may assume that $\left|u_{j}\right|=1$. We first consider the case when $M \otimes E$ has pure visible intrinsic $\partial_{j}$-radii $I R_{\partial_{j}}(M \otimes E)<\omega$. By making $\alpha$ closer to $1^{-}$, we may assume that the function $f_{i}^{(j)}(M, r)>-\log \omega$ for each $i$ is affine over $[0,-\log \alpha)$.

As in Theorem 4.1.9, we may identify $M \otimes \mathscr{R}_{K}^{\mathrm{bd}}$ with $\mathscr{R}^{\mathrm{bd}}\{T\} / \mathscr{R}^{\mathrm{bd}}\{T\} P$ for some twisted polynomial $P=T^{d}+a_{1} T^{d-1}+\cdots+a_{d} \in \mathscr{R}^{\mathrm{bd}}\{T\}$. Since $M \otimes E$ has pure $\partial_{j}$-radii $\omega \mathrm{e}^{s}$, the Newton polygon of $P$ with respect to the 1 -Gauss norm has pure 
slope $s$ and the multiset $\Theta_{\partial_{j}}(M \otimes E)$ is just the multiset of reduced roots of this twisted polynomial. We put

$$
\bar{P}=T^{d}+\bar{a}_{1}^{(s)} T^{d-1}+\cdots+\bar{a}_{d}^{(d s)},
$$

where $\bar{a}_{i}^{(i s)} \in \kappa_{K}^{(i s)}((t))$.

When $\eta$ is sufficiently close to $1^{-}$, the Newton polygon of $P$ with respect to the $\eta$-Gauss norm is determined by the Newton polygon of $\bar{P}$ in the following sense: it is the lower convex hull of the set $\left\{\left(-i,-\log \left|a_{i}\right|_{1}-\mathrm{v}\left(\bar{a}_{i}^{(i s)}\right) \log \eta\right)\right\}$. By Lemma 4.1.8, this implies that the collection of all slopes of functions $f_{i}^{(j)}(M, r)$ for all $i$ at $r=0$ is exactly the collection of the $v_{s}$-valuations of the roots of $\bar{P}$, which in turn equals the collection of the $v_{s}$-valuations of the elements of the multiset of refined $\partial_{j}$-radii of $M \otimes E$.

Now, it suffices to reduce to the case above using $\partial_{j}$-Frobenius. Assume $p>0$ from now on. It is easier to work with intrinsic radii and refined intrinsic radii. So we put $g_{i}(M, r)=f_{i}^{(j)}(M, r)+\log \left|u_{j}\right|$ if $j \in J$ and $g_{i}(M, r)=f_{i}^{(j)}(M, r)-r$ if $j=0$. We will use $g_{i}^{\prime}(M, \cdot)$ to denote the derivative of the function $g_{i}(M, \cdot)$. Moreover, we set $s^{\prime}=-\log \left(\omega I R_{\partial_{j}}(V)^{-1}\right)$.

If $I R_{\partial_{j}}(M \otimes E)=\omega=p^{-1 /(p-1)}$, we set $M_{1}=\varphi_{*}^{\left(\partial_{j}\right)} M$. Then Lemma 1.2.18(d) implies that if $j \in J$,

$$
\left\{g_{i}^{\prime}\left(M_{1}, 0\right)\right\}= \begin{cases}\left\{p g_{i}^{\prime}(M, 0)(d \text { times }), 0((p-1) d \text { times })\right\} & \text { if } g_{i}^{\prime}(M, 0)<0, \\ \left\{g_{i}^{\prime}(M, 0)(p d \text { times })\right\} & \text { if } g_{i}^{\prime}(M, 0) \geq 0,\end{cases}
$$

and if $j=0$,

$$
\left\{g_{i}^{\prime}\left(M_{1}, 0\right)\right\}= \begin{cases}\left\{g_{i}^{\prime}(M, 0), 0(p-1 \text { times })\right\} & \text { if } g_{i}^{\prime}(M, 0)<0, \\ \left\{\frac{1}{p} g_{i}^{\prime}(M, 0)(p \text { times })\right\} & \text { if } g_{i}^{\prime}(M, 0) \geq 0 .\end{cases}
$$

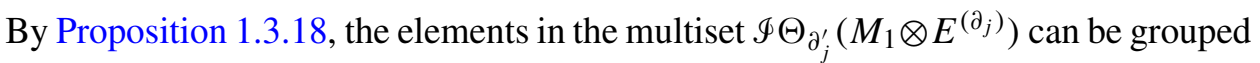
into $p$-tuples

$$
\left(\frac{\theta}{p}, \frac{\theta+1}{p}, \ldots, \frac{\theta+p-1}{p}\right),
$$

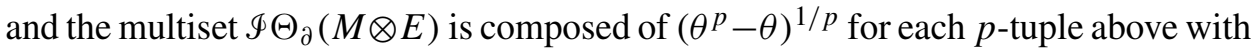
the same multiplicity, where $\theta \in \kappa_{E^{\text {alg }}}$. Elementary calculation shows the following

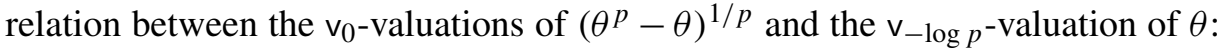

- when $\mathrm{v}_{0}(\theta)<0$, we have $\mathrm{v}_{-\log } p((\theta+l) / p)=\mathrm{v}_{0}(\theta)$ for $l=0, \ldots, p-1$, and $\mathrm{v}_{0}\left(\left(\theta^{p}-\theta\right)^{1 / p}\right)=\mathrm{v}_{0}(\theta)$;

- when $\mathrm{v}_{0}(\theta) \geq 0$, we have $\mathrm{v}_{-\log p}((\theta+l) / p)=0$ for $l=1, \ldots, p-1$, and $\mathrm{v}_{0}\left(\left(\theta^{p}-\theta\right)^{1 / p}\right)=(1 / p) \mathrm{v}_{0}(\theta)$.

Hence the statement for $M_{1}$ with $\vee_{-\log p}$ implies that for $M$ with $\vee_{0}$. 
If $I R_{\partial_{j}}(M \otimes E)>\omega$, by Lemma 1.2.18(d) and Remark 1.2.19, $M$ has a $\partial_{j}$ Frobenius antecedent $M_{0}$ if $\alpha$ is sufficiently close to $1^{-}$. By Lemma 1.2.18(d) and Proposition 1.3.18, we have

$g_{i}\left(M_{0}, r\right)=p g_{i}(M, r)$ for any $i$,

and $\mathscr{I} \Theta_{\partial_{j}^{\prime}}\left(M_{0} \otimes E^{\left(\partial_{j}\right)}\right)=\left\{(-\theta)^{p} / p: \theta \in \mathscr{I} \Theta_{\partial_{j}}(M \otimes E)\right\}$, if $j \in J$; $g_{i}\left(M_{0}, p r\right)=p g_{i}(M, r)$ for any $i$, and $\mathscr{I} \Theta_{\partial_{j}^{\prime}}\left(M_{0} \otimes E^{\left(\partial_{j}\right)}\right)=\left\{(-\theta)^{p} / p: \theta \in \mathscr{I} \Theta_{\partial_{j}}(M \otimes E)\right\}$, if $j=0$.

Since $\mathrm{v}_{\left(p s^{\prime}-\log p\right)}\left((-\theta)^{p} / p\right)=p \mathrm{v}_{s^{\prime}}(\theta)$, the statement for $M$ with $\mathrm{v}_{s^{\prime}}(-\log p)$ follows from the statement for $M_{0}$ with $\vee_{p s^{\prime}-\log p}$ if $j \in J$ and with $\frac{1}{p} \vee_{p s^{\prime}-\log p}$ if $j=0$ (note that $t^{p}$ is the coordinate in the latter case).

Corollary 4.2.2. Fix $j \in J^{+}$and let $M$ be a $\partial_{j}$-differential module over $K\left\{\left\{\alpha / t, t \rrbracket_{0}\right.\right.$. Assume that $M \otimes E$ has pure $\partial_{j}$-radii $R_{\partial_{j}}(M \otimes E)=\omega \mathrm{e}^{s}$, which is strictly less than $\left|u_{j}\right|^{-1}$ if $j \in J$ and is strictly less than 1 if $j=0$. Then the following two multisets are the same:

(i) the multiset composed of the $\mathrm{v}_{s}$-valuations of the elements in the multiset of refined $\partial_{j}$-radii of $M \otimes E$, that is, $\left\{\mathrm{v}_{s}(\theta): \theta \in \Theta_{\partial_{j}}(M \otimes E)\right\}$;

(ii) the multiset composed of the negatives of the slopes of $f_{i}^{(j)}(M, r)$ at $r=0$, for $i=1, \ldots, d$.

Proof. This follows from combining Theorems 4.1.9 and 4.2.1.

Notation 4.2.3. For any $\mathfrak{s} \in \mathbb{Q} \cdot \log \left|K^{\times}\right|$, the valuation $v_{\mathfrak{s}}$ on $\kappa_{E}^{(\mathfrak{s})}$ induces a valuation on

$$
\kappa_{E}^{(\mathfrak{s})} \frac{d t}{t} \oplus \bigoplus_{j \in J} \kappa_{E}^{(\mathfrak{s})} \frac{d u_{j}}{u_{j}},
$$

still denoted by $v_{\mathfrak{s}}$, by setting

$$
\mathrm{v}_{\mathfrak{s}}\left(\theta_{0} \frac{d t}{t}+\theta_{1} \frac{d u_{1}}{u_{1}}+\cdots+\theta_{m} \frac{d u_{m}}{u_{m}}\right)=\min _{j \in J^{+}}\left\{\mathrm{v}_{\mathfrak{s}}\left(\theta_{j}\right)\right\}, \text { for } \theta_{0}, \ldots, \theta_{m} \in \kappa_{E}^{(\mathfrak{s})} .
$$

Corollary 4.2.4. Let $M$ be a $\partial_{J^{+}}$-differential module over $K\left\{\left\{\alpha / t, t \rrbracket_{0}\right.\right.$. Assume that $M \otimes E$ has pure intrinsic radii $I R(M \otimes E)=\omega \mathrm{e}^{\mathfrak{s}}<1$. Then the following two multisets are the same:

(i) the valuations of the refined intrinsic radii of $M \otimes E,\left\{\mathrm{v}_{\mathfrak{s}}(\theta): \theta \in \mathscr{I} \Theta(M \otimes E)\right\}$;

(ii) the negatives of the slopes of $f_{i}(M, r)$ at $r=0$, for $i=1, \ldots, d$.

Proof. This follows from combining Theorems 4.1.9 and 4.2.1.

Similar to Theorem 1.3.26, we have the following decomposition by refined radii. 
Theorem 4.2.5. Fix $j \in J^{+}$and let $M$ be a $\partial_{j}$-differential module of rank $d$ over $K\left\{\left\{\alpha / t, t \rrbracket_{0}\right.\right.$. Assume that $M \otimes F_{\eta}$, for $\eta \in(\alpha, 1)$, and $M \otimes E$ all have pure $\partial_{j}$-radii, and assume that the function $f_{1}^{(j)}(M, r)$ is affine with slope $b$ for $r \in[0,-\log \alpha)$. Let $e$ be the prime-to- $p$ part of the denominator of $b$. Moreover, assume that $R_{\partial_{j}}(M \otimes E)=\omega \mathrm{e}^{s}$ is strictly less than $\left|u_{j}\right|^{-1}$ if $j \in J$ and is strictly less than 1 if $j=0$. Then there exists a finite tamely ramified extension $K^{\prime}$ of $K$ and a unique direct sum decomposition

$$
M \otimes K^{\prime}\left\{\left\{\alpha^{1 / e} / t^{1 / e}, t^{1 / e} \rrbracket_{0}=\bigoplus_{\theta \in \kappa_{K^{\text {alg }}}^{(s)}} M_{\theta}\right.\right.
$$

of $\partial_{j}$-differential modules such that

(i) $M_{\theta} \otimes F_{\eta}$ has pure refined $\partial_{j}$-radii $\theta t^{-b}$ for all $\eta \in(\alpha, 1)$, and

(ii) every element in the multiset of refined $\partial_{j}$-radii of $M_{\theta} \otimes E$ is congruent to $\theta t^{-b}$ modulo elements in $\kappa_{K}^{(s)}$ alg with $\mathrm{v}_{s}$-valuation strictly bigger than $\mathrm{v}_{s}\left(\theta t^{-b}\right)=-b$.

Moreover, this decomposition descents to a unique decomposition of $M$ itself by Galois descent, satisfying analogous properties, but in the fashion stated in terms of $\mu_{e} \rtimes \operatorname{Gal}\left(K^{\mathrm{alg}} / K\right)$-orbits.

Proof. The proof is identical to that of Theorem 1.5.10, except that we use decomposition Theorem 4.1.9 in place of Theorem 1.5.4.

Theorem 4.2.6. Let $M$ be a $\partial_{J^{+}}$-differential module of rank $d$ over $K\left\{\left\{\alpha / t, t \rrbracket_{0}\right.\right.$. Assume that $M \otimes F_{\eta}$, for $\eta \in(\alpha, 1)$, and $M \otimes E$ all have pure intrinsic radii, and assume that the function $f_{1}(M, r)$ is affine with slope $b$ for $r \in[0,-\log \alpha)$. Let $e$ be the prime-to- $p$ part of the denominator of $b$. Moreover, assume that $\operatorname{IR}(M \otimes E)=\omega \mathrm{e}^{\mathfrak{s}}<1$. Then there exists a finite tamely ramified extension $K^{\prime}$ of $K$ and a unique direct sum decomposition

$$
M \otimes K^{\prime}\left\{\left\{\alpha^{1 / e} / t^{1 / e}, t^{1 / e} \rrbracket_{0}=\bigoplus_{\vartheta \in \bigoplus_{j \in J} \kappa_{K^{\text {alg }}}^{(\mathfrak{s})} \frac{d u_{j}}{u_{j}} \oplus \kappa_{K^{\text {alg }}}^{(\mathfrak{s})} \frac{d t}{t}} M_{\vartheta}\right.\right.
$$

of $\partial_{J^{+}-\text {differential modules such that }}$

(i) $M_{\vartheta} \otimes F_{\eta}$ has pure refined intrinsic radii $\vartheta t^{-b}$ for all $\eta \in(\alpha, 1)$, and

(ii) every element in the multiset of refined intrinsic radii of $M_{\vartheta} \otimes E$ is congruent to $\vartheta t^{-b}$ modulo those elements in

$$
\bigoplus_{j \in J} \kappa_{K^{\text {alg }}}^{(\mathfrak{s})} \frac{d u_{j}}{u_{j}} \oplus \kappa_{K^{\text {alg }}}^{(\mathfrak{s})} \frac{d t}{t}
$$

with $\mathrm{v}_{\mathfrak{s}}$-valuation strictly bigger than $\mathrm{v}_{\mathfrak{s}}\left(\vartheta t^{-b}\right)=-b$. 
Moreover, this decomposition descents to a unique decomposition of $M$ itself by Galois descent, satisfying analogous properties, but in the fashion stated in terms of $\mu_{e} \rtimes \operatorname{Gal}\left(K^{\mathrm{alg}} / K\right)$-orbits.

Proof. The proof is identical to that of Theorem 1.5.12, except that we invoke Theorem 4.2.5 in place of Theorem 1.5.4.

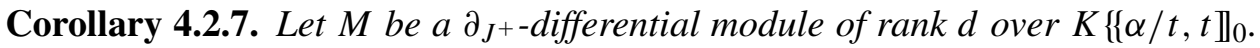
Assume that $M \otimes E$ has pure intrinsic radii $\operatorname{IR}(M \otimes E)=\omega \mathrm{e}^{\mathfrak{s}}<1$ and that the function $f_{i}(M, r)$ for each $i=1, \ldots, d$ is affine over $[0,-\log \alpha)$. Let $M=\bigoplus_{b \in \mathbb{Q}} M_{b}$ be the unique direct sum decomposition of $M$ over $A_{K}^{1}(\alpha, 1)$ such that $f_{1}\left(M_{b}, r\right)=$ $\cdots=f_{\operatorname{dim} M_{b}}\left(M_{b}, r\right)$ has slope $b$. Then the following two multisets are the same:

(i) The multiset composed of all elements in

$$
g \Theta\left(M_{b} \otimes F_{\eta}\right) \subset \bigoplus_{j \in J^{+}} t^{-b} \kappa_{K^{\mathrm{alg}}}^{(\mathfrak{s})} \frac{d u_{j}}{u_{j}} \oplus t^{-b} \kappa_{K^{\mathrm{alg}}}^{(\mathfrak{s})} \frac{d t}{t}
$$

for all $b$ and for some fixed $\eta \in(\alpha, 0)$ (this is independent of the choice of $\eta$ );

(ii) The multiset composed of $\bar{\vartheta}$ for all $\vartheta \in \Theta_{\partial_{j}(V)}$, where $\bar{\vartheta}$ is the reduction of

$$
\vartheta \in \bigoplus_{j \in J^{+}} t^{-b} \kappa_{K^{\mathrm{alg}}}^{(\mathfrak{s})} \frac{d u_{j}}{u_{j}} \oplus t^{-b} \kappa_{K^{\mathrm{alg}}}^{(\mathfrak{s})} \frac{d t}{t}
$$

modulo those elements with $\mathrm{v}_{S}$-valuation strictly bigger than $\mathrm{v}_{S}(\vartheta)$.

Proof. It follows from the decomposition Theorems 4.1.9 and 4.2.6.

We have similar results for extrinsic radii.

Theorem 4.2.8. Assume that $\left|u_{j}\right|=1$ for all $j \in J$. For $s \in \mathbb{R}$, let $\hat{v}_{s}$ be the valuation on $\kappa_{E}^{(s)} d t \oplus \bigoplus_{j \in J} \kappa_{E}^{(s)} d u_{j}$ given by

$$
\hat{\mathrm{v}}_{s}\left(\theta_{0} d t+\theta_{1} d u_{1}+\cdots+\theta_{m} d u_{m}\right)=\min _{j \in J^{+}}\left\{\mathrm{v}_{s}\left(\theta_{j}\right)\right\} .
$$

Let $M$ be a $\partial_{J^{+}}$-differential module of rank $d$ over $K\left\{\left\{\alpha / t, t \rrbracket_{0}\right.\right.$. Assume that $M \otimes F_{\eta}$, for $\eta \in(\alpha, 1)$, and $M \otimes E$ all have pure extrinsic radii, and assume that the function $\hat{f}_{1}(M, r)$ is affine with slope $b$ for $r \in[0,-\log \alpha)$. Let e be the prime-to-p part of the denominator of $b$. Moreover, assume that $E R(M \otimes E)=\omega \mathrm{e}^{s}<1$. Then there exists a unique direct sum decomposition $M=\bigoplus_{\left\{\mu_{e} \hat{\vartheta}\right\}} M_{\left\{\mu_{e} \hat{\vartheta}\right\}}$ of $\partial_{J^{+-} \text {differential modules }}$ over $K\left\{\left\{\alpha / t, t \rrbracket_{0}\right.\right.$, where the direct sum runs through all $\mu_{e} \rtimes \operatorname{Gal}\left(K^{\text {sep }} / K\right)$-orbits $\left\{\mu_{e} \hat{\vartheta}\right\}$ in $\bigoplus_{j \in J} \kappa_{K^{\text {alg }}}^{(s)} d u_{j} \oplus \kappa_{K^{\text {alg }}}^{(s)} d t$ such that

(i) for all $\eta \in(\alpha, 1)$, the multiset of refined extrinsic radii of $M_{\left\{\mu_{e} \vartheta\right\}} \otimes F_{\eta}$ is composed of the $\mu_{e} \rtimes \operatorname{Gal}\left(K^{\mathrm{alg}} / K\right)$-orbit $\left\{\mu_{e} \hat{\vartheta} t^{-b}\right\}$ with the appropriate multiplicity, and 
(ii) the multiset consisting of the reductions of elements in the multiset of refined extrinsic radii of $M_{\left\{\mu_{e} \hat{\vartheta}\right\}} \otimes E$ modulo those elements with $\hat{\mathrm{v}}_{s}$-valuation is strictly bigger than $-b$, is composed of the $\mu_{e} \rtimes \operatorname{Gal}\left(K^{\mathrm{alg}} / K\right)$-orbit $\left\{\mu_{e} \hat{\vartheta} t^{-b}\right\}$ with the appropriate multiplicity.

Proof. The proof is identical to that of Theorem 1.5.14, except that we use invoke Theorem 4.2.5 in place of Theorem 1.5.4.

Corollary 4.2.9. Assume that $\left|u_{j}\right|=1$ for all $j \in J$. Let $M$ be a $\partial_{J^{+}-\text {differential }}$ module of rank $d$ over $K \llbracket t \rrbracket_{0}$. Assume that $E R(M \otimes E)=\omega \mathrm{e}^{s}<1$. Let $M_{e}$ denote the unique $\partial_{J^{+}}$-differential submodule of $M \otimes E$ that has pure extrinsic radii $E R(M \otimes E) ;$ put $l=\operatorname{dim} M_{e}$. Then:

(a) The $\hat{v}_{s}$-valuations of elements in $\Theta\left(M_{e} \otimes E\right)$ are all nonnegative.

(b) There exists a unique direct sum decomposition

$$
M=\bigoplus M_{\{\hat{\vartheta}\}} \oplus M_{0}
$$

$\{\hat{\vartheta}\}$

of $\partial_{J^{+}}$-differential modules over $K \llbracket t \rrbracket_{0}$, where the first direct sum is taken over all $\operatorname{Gal}\left(\kappa_{K}^{\text {sep }} / \kappa_{K}\right)$-orbits $\{\hat{\vartheta}\} \subset \bigoplus_{j \in J} \kappa_{K^{\text {alg }}}^{(s)} d u_{j} \oplus \kappa_{K^{\text {alg }}}^{(s)} d t$ such that

(i) for all $\eta<1, M_{\{\hat{\vartheta}\}} \otimes F_{\eta}$ has pure extrinsic radii $\min \left\{\omega \mathrm{e}^{s}, \eta\right\}$ and, when $\eta \in$ $\left(\omega \mathrm{e}^{s}, 1\right)$, the multiset $\Theta\left(M_{\{\vartheta\}} \otimes F_{\eta}\right)$ is composed of $\{\hat{\vartheta}\}$ with multiplicity,

(ii) the multiset consisting of reductions of elements in the multiset of refined extrinsic radii of $M_{\{\vartheta\}} \otimes E$ modulo those elements with positive $\hat{v}_{s}$-valuation is composed of $\{\hat{\vartheta}\}$ with appropriate multiplicity, and

(iii) for any $r>0$ satisfying $\hat{f}_{1}\left(M_{0}, r\right)<r$, we have $\hat{f}_{1}\left(M_{0}, r\right)<\omega \mathrm{e}^{s}$.

Proof. (a) By Proposition 4.1.11(a) together with Theorem 1.5.6( $\left.\mathrm{c}^{\prime}\right)$, we know that the functions $f_{1}(M, r), \ldots, f_{l}(M, r)$ are linear in a neighborhood of $r$ with nonpositive slopes. Then applying the decomposition in Proposition 4.1.11(b) and Theorem 4.2.8 together with description (ii) in Theorem 4.2.8, we conclude that the $\hat{v}_{s}$-valuations of elements in $\Theta\left(M_{e}\right)$ are all nonnegative.

(b) Let $l^{\prime}$ denote the number of elements in $\Theta\left(M_{e}\right)$ whose $\hat{v}_{s}$-valuation is zero. By the proof of (a), we see that the derivatives $\hat{f}_{1}^{\prime}(M, 0)=\cdots=\hat{f}_{l^{\prime}}^{\prime}(M, 0)$ are equal to 0 , and that $f_{l^{\prime}+1}^{\prime}(M, 0)>0$ or $\hat{f}_{l^{\prime}+1}(M, 0)>\hat{f}_{l}(M, 0)$ in case $l=l^{\prime}$. By items $\left(\mathrm{c}^{\prime}\right)$ and $(\mathrm{d})$ of Theorem 1.5.6, we know that

$$
\hat{f}_{1}(M, 0)=\hat{f}_{1}(M, r)=\cdots=\hat{f}_{l^{\prime}}(M, r)>\hat{f}_{l^{\prime}+1}(M, r)
$$

for any $r<\hat{f}_{1}(M, 0)$. We may then apply Proposition 4.1.11 to split off the desired $M_{0}$. Now, we may apply the standard technique (Lemma 1.1.10 and Remark 1.1.11) to glue the decomposition given by Theorem 4.2.8 and Proposition 1.5.17; this gives the further decompositions by $M_{\{\theta\}}$. 
4.3. Variation over polyannuli. In this subsection, we study differential modules over a polyannulus or a polydisc. In particular, we are interested in studying the functions coming from the radii of convergence when we complete the differential module with respect to various Gauss norms. We relate the slopes of such functions with the valuations of the refined intrinsic radii.

In this subsection, we assume Hypothesis 1.5.1 and we assume that $K$ is discretely valued.

Definition 4.3.1. A subset $C \subseteq \mathbb{R}^{n}$ is called nondegenerate if it contains an open subset of $\mathbb{R}^{n}$. Its interior is denoted by $C^{\text {int }}$.

An integral affine functional on $\mathbb{R}^{n}$ is a map $\lambda: \mathbb{R}^{n} \rightarrow \mathbb{R}$ of the form

$$
\lambda\left(x_{1}, \ldots, x_{n}\right)=a_{1} x_{1}+\cdots+a_{n} x_{n}+b
$$

for some $a_{1}, \ldots, a_{n} \in \mathbb{Z}$ and $b \in-\log \left|K^{\times}\right|^{\mathbb{Q}}$.

A subset $C \subseteq \mathbb{R}^{n}$ is rational polyhedral (or $R P$ for short) if it is bounded and there exist integral affine functionals $\lambda_{1}, \ldots, \lambda_{r}$ such that

$$
C=\left\{x \in \mathbb{R}^{n}: \lambda_{i}(x) \geq 0 \text { for } i=1, \ldots, r\right\} .
$$

For $C \subseteq \mathbb{R}^{n}$ an RP subset of $\mathbb{R}^{n}$, a function $f: C \rightarrow \mathbb{R}^{n}$ is integral polyhedral if there exist finitely many integral affine functionals $\lambda_{1}^{\prime}, \ldots, \lambda_{d}^{\prime}$ such that $f(x)=\max \left\{\lambda_{1}^{\prime}(x), \ldots, \lambda_{d}^{\prime}(x)\right\}$ for any $x \in C$.

Remark 4.3.2. Our convention slightly differs from that of [Kedlaya and Xiao 2010], where RP subsets are not assumed to be bounded. However, some of the statements below still hold for unbounded RP, and they are often simple corollaries of the statements in the bounded case. We leave this as an exercise for the reader.

Notation 4.3.3. We put $I=\{1, \ldots, n\}$. We use $\underline{a}$ to denote the $n$-tuple $(a, \ldots, a)$.

Definition 4.3.4. For a subset $C \subseteq \mathbb{R}^{n}$, let $\mathrm{e}^{-C}$ denote the closure in $\mathbb{R}^{n}$ of the subset $\left\{\mathrm{e}^{-r_{I}}: r_{I} \in C\right\}$. A subset $S$ of $[0,+\infty)^{n}$ is called $\log -R P$ if $S=\mathrm{e}^{-C}$ for some RP subset $C$ of $\mathbb{R}^{n}$; it is called nondegenerate if $C$ is so.

For $S$ a log-RP subset of $[0,+\infty)^{n}$, define $A_{K}\left(S^{\text {int }}\right)$ to be the subspace of the (Berkovich) analytic $n$-space with coordinates $t_{1}, \ldots, t_{n}$ satisfying the condition $\left(\left|t_{1}\right|, \ldots,\left|t_{n}\right|\right) \in \mathrm{e}^{-C^{\text {int }}}$. We use $K\{\{S\}\}$ to denote its ring of functions, and use $K \llbracket S \rrbracket_{0}$ to denote the subring of $K\{\{S\}\}$ consisting of functions that are bounded on $\left|t_{I}\right| \in \mathrm{e}^{-C^{\text {int }}}$.

One cannot literally equate $S^{\text {int }}$ with $\mathrm{e}^{-C^{\text {int }}}$; the problem is that we cannot take the $\log$ for a zero coordinate in $S$-space. But, in practice, one can view the two spaces the same, just being careful when stating a result.

Notation 4.3.5. Let $S$ be a nondegenerate log-RP subset of $[0,+\infty)^{n}$ and let $R$ denote either $K\{\{S\}\}$ or $K \llbracket S \rrbracket_{0}$. Let $M$ be a $\partial_{I \cup J}$-differential module over $R$ of rank 
$d$, with respect to the derivations $\partial_{1}, \ldots, \partial_{m}$ and $\partial_{m+1}=\partial / \partial t_{1}, \ldots, \partial_{m+n}=\partial / \partial t_{n}$. For an element $\eta_{I}$ in $\left(\eta_{1}, \ldots, \eta_{n}\right) \in S$ ( $S^{\text {int }}$ if $\left.R=K\{\{S\}\}\right)$, let $F_{\eta_{I}}$ be the completion of $\operatorname{Frac}(R)$ with respect to the $\eta_{I}$-Gauss norm. We remark that for $\eta_{I}$ on the boundary of $S, F_{\eta_{I}}$ "looks different" (more like $E$ than $F_{\eta}$ in the 1-dimensional case).

For an element $r_{I}$ in $-\log S$ (or $-\log S^{\text {int }}$ if $\left.R=K\{\{S\}\}\right)$, put

$f_{l}\left(M, r_{I}\right)=-\log I R\left(M \otimes F_{\mathrm{e}^{-r_{I}}} ; l\right) \quad$ and $\quad F_{l}\left(M, r_{I}\right)=f_{1}\left(M, r_{I}\right)+\cdots+f_{l}\left(M, r_{I}\right)$ for $l=1, \ldots, d$.

Theorem 4.3.6. Keep the notation as above. We have the following:

(a) (Polyhedrality) The functions $d ! F_{l}\left(M, r_{I}\right)$, for $l=1, \ldots, d-1$, and $F_{d}\left(M, r_{I}\right)$ are integral polyhedral functions.

(b) (Decomposition) Suppose that for some $l \in\{1, \ldots, d\}$, the function $F_{l}\left(M, r_{I}\right)$ is affine, and suppose that $f_{l}\left(M, r_{I}\right)>f_{l+1}\left(M, r_{I}\right)$ for any $r_{I} \in-\log S$. Then $M$ admits a unique direct sum decomposition $M \cong M_{0} \oplus M_{1}$ of differential modules such that for any $\eta_{I} \in-\log S^{\text {int }}$, the multiset of intrinsic radii of $M_{0}$ exactly consists of the smallest l elements in the multiset of intrinsic radii of $M \otimes F_{\eta_{I}}$.

(c) (Refined radii) Assume that $R=K\{\{S\}\}$ and that

$$
f_{1}\left(M, r_{I}\right)=\cdots=f_{d}\left(M, r_{I}\right)=-\log \omega-\mathfrak{s}+b_{1} r_{1}+\cdots+b_{n} r_{n}
$$

are affine functions on $-\log S^{\mathrm{int}}$. Let $e_{i}$ denote the prime-to- $p$ part of the denominator of $b_{i}$ for all $i \in I$. Then there exists a finite tamely ramified extension $K^{\prime}$ of $K$ and a multiset

$$
g \Theta(M) \subset \bigoplus_{i \in I} \kappa_{K^{\prime}}^{(\mathfrak{s})} \frac{d t_{i}}{t_{i}} \bigoplus \bigoplus_{j \in J} \kappa_{K^{\prime}}^{(\mathfrak{s})} \frac{d u_{j}}{u_{j}}
$$

such that we have a unique direct sum decomposition of differential modules

$$
M \otimes_{R} R\left[t_{1}^{1 / e_{1}}, \ldots, t_{n}^{1 / e_{n}}\right]=\bigoplus_{\vartheta \in \mathscr{I} \Theta(M)} M_{\vartheta},
$$

such that each $M_{\vartheta} \otimes F_{\eta_{I}}\left[t_{1}^{1 / e_{1}}, \ldots, t_{n}^{1 / e_{n}}\right]$ has pure refined intrinsic radii $t_{I}^{-b_{I}} \vartheta$. Proof. For (a) and (b), see [Kedlaya and Xiao 2010, Theorems 3.3.9 and 3.4.4, and Remark 3.4.7]. (c) follows from the same argument but using Theorem 1.5.12 as the decomposition tool.

To extend (c) of the theorem above to the boundary is a little tricky. We will prove it in a special case and leave the general case as an exercise for the reader.

Situation 4.3.7. Consider the subset $C=\left\{\left(x_{I}\right) \subset \mathbb{R}^{n}: x_{I} \geq 0, x_{1}+\cdots+x_{n} \leq 1\right\}$. Put $S=\mathrm{e}^{-C}$, and $R=K \llbracket S \rrbracket_{0}$. Let $M$ be a differential module over $K \llbracket S \rrbracket_{0}$. Assume 
moreover that $f_{1}(M, \underline{0})=\cdots=f_{d}(M, \underline{0})=-\log \omega-\mathfrak{s}$ with $\mathfrak{s}<0$. We define the following two multisets.

(1) Choose $x \in \mathfrak{m}_{K}^{(\mathfrak{s})} \backslash \mathfrak{m}_{K}^{(\mathfrak{s})+}$ to identify $\kappa_{F_{1}}^{(\mathfrak{s})} \stackrel{\cdot x^{-1}}{\longrightarrow} \kappa_{F_{1}}$ and embed the latter into the higher local field $\kappa_{K}\left(\left(t_{1}\right)\right) \cdots\left(\left(t_{n}\right)\right)$, which is equipped with a multi-indexed valuation with respect to the parameters $\left(t_{n}, \ldots, t_{1}\right)$. This gives rise to a valuation $\boldsymbol{v}_{\mathfrak{s}}: \kappa_{F_{1}}^{(\mathfrak{s})} \rightarrow \mathbb{Z}^{n} \subset \mathbb{Q}^{n}$, where the latter is equipped with the lexicographical order; this does not depend on the choice of $x$. Define the following valuation on

$$
\bigoplus_{i \in I} \kappa_{F_{1}}^{(\mathfrak{s})} \frac{d t_{i}}{t_{i}} \oplus \bigoplus_{j \in J} \kappa_{F_{1}^{\mathrm{alg}}}^{(\mathfrak{s})} \frac{d u_{j}}{u_{j}}
$$

still denoted by $\boldsymbol{v}_{\mathfrak{s}}$, by taking the minimum of $\boldsymbol{v}_{\mathfrak{s}}$ over the coefficients. We consider the multiset $A=\left\{(\boldsymbol{v}(\vartheta), \bar{\vartheta}) \mid \vartheta \in \mathscr{I} \Theta\left(M \otimes F_{1}\right)\right\}$, where $\bar{\vartheta}$ is the reduction of $t_{I}^{-v_{\mathfrak{s}}(\vartheta)} \vartheta$ to

$$
\bigoplus_{i \in I} \kappa_{K^{\mathrm{alg}}}^{(\mathfrak{s})} \frac{d t_{i}}{t_{i}} \oplus \bigoplus_{j \in J} \kappa_{K^{\mathrm{alg}}}^{(\mathfrak{s})} \frac{d u_{j}}{u_{j}}
$$

(2) By Theorem 4.3.6(a), there exists an RP subset $C^{\prime}$ of $C$ which is adjacent to the cells $t_{1}=\cdots=t_{i}=0$ for $i=1, \ldots, n-1$, such that the function $f_{l}\left(M, r_{I}\right)$ for each $l$ is affine in $r_{I}$ over $C^{\prime}$. Then, over $\mathrm{e}^{-C^{\text {int }}}$, we have a unique direct sum decomposition of differential modules $M=\bigoplus_{b_{I} \in \mathbb{Q}^{n}} M_{b_{I}}$ such that $f_{1}\left(M_{b_{I}}, r_{I}\right)=\cdots=f_{\operatorname{dim} M_{b_{I}}}\left(M_{b_{I}}, r_{I}\right)=-\log \omega-\mathfrak{s}+b_{1} r_{1}+\cdots+b_{n} r_{n}$.

We put

$$
B=\left\{\left(-b_{1}, \ldots,-b_{n}, \vartheta\right): b_{I} \in \mathbb{Q}^{n}, t_{1}^{-b_{1}} \cdots t_{n}^{-b_{n}} \vartheta \in \mathscr{I} \Theta\left(M \otimes F_{\eta_{I}}\right)\right\}
$$

for some $\eta_{I} \in C^{\text {int }}$ and this set does not depend on the choice of $\eta_{I}$ by Theorem 4.3.6(c).

Choose integers $e_{1}, \ldots, e_{n} \in \mathbb{N}$ coprime to $p$ such that $e_{i} b_{i} \in \mathbb{Z}$ for any $i$ and for any $\left(-b_{1}, \ldots,-b_{n}, \vartheta\right) \in B$. Put $R^{\prime}=K \llbracket C^{\prime} \rrbracket_{0}\left[t_{1}^{1 / e_{1}}, \ldots, t_{n}^{1 / e_{n}}\right]$.

Theorem 4.3.8. The two multisets $A$ and $B$ are the same (for any $C^{\prime}$ that satisfies the condition in (2)). Moreover, there exists a finite tamely ramified extension $K^{\prime} / K$ and a unique direct sum decomposition

$$
M \otimes R^{\prime} \otimes K^{\prime}=\bigoplus_{\left(b_{I}, \vartheta\right) \in B} M_{\left(b_{I}, \vartheta\right)}
$$

such that, if we put $F_{\mathrm{e}^{-r_{I}}}^{\prime}=F_{\mathrm{e}^{-r_{I}}}\left[t_{1}^{1 / e_{1}}, \ldots, t_{n}^{1 / e_{n}}\right] \otimes K^{\prime}$, 
(i) for all $r_{I} \in C^{\text {int }}, M_{\left(b_{I}, \vartheta\right)} \otimes F_{\mathrm{e}^{-r_{I}}}^{\prime}$ has pure intrinsic radii $\omega \mathrm{e}^{-b_{1} r_{1}-\cdots-b_{n} r_{n}+\mathfrak{s}}$ and pure refined intrinsic radii $t_{I}^{-b_{I}} \vartheta$, and

(ii) any element in $\Phi \Theta\left(M \otimes F_{1}^{\prime}\right)$ is congruent to $t_{I}^{-b_{I}} \vartheta$ modulo elements with $\boldsymbol{v}_{s}$-valuation strictly bigger than $\left(-b_{1}, \ldots,-b_{n}\right)$.

Proof. We first construct the decomposition that satisfies condition (i). For this, we may replace $K$ by a finite tamely ramified extension such that all $\vartheta$ appearing in $B$ lie in

$$
\bigoplus_{i \in I} \kappa_{K}^{(s)} \frac{d t_{i}}{t_{i}} \oplus \bigoplus_{j \in J} \kappa_{K}^{(s)} \frac{d u_{j}}{u_{j}}
$$

for an appropriate $s$. In this case, we construct the decomposition of $M \otimes R^{\prime}$ using the same argument as in [Kedlaya and Xiao 2010, Theorem 3.4.4] by invoking Theorems 4.1.9 and 4.2.6 at appropriate places.

Now we check condition (ii) for this direct sum decomposition; this is equivalent to identifying the multisets A with B for each $M_{b_{I}, \vartheta}$. Note that we already know that $M_{b_{I}, \vartheta} \otimes F_{e^{-r_{I}}}$ has pure intrinsic radii $\omega e^{-b_{1} r_{1}-\cdots-b_{n} r_{n}+s}$. For simplicity, we put $M=M_{b_{I}, \vartheta}$. We do induction on the dimension $n$. When $n=0$ there is nothing to prove. We assume that the theorem is proved for $n-1$. Let $D$ denote the face $t_{1}=0$ of $C$. Put $\widetilde{C}=C \cap D, \widetilde{C}^{\prime}=C^{\prime} \cap D, \widetilde{S}=\mathrm{e}^{-\widetilde{C}}$, and $\widetilde{R}=\widetilde{K} \llbracket \widetilde{S} \rrbracket_{0}$ with coordinates $t_{2}, \ldots, t_{n}$, where $\widetilde{K}$ is the completion of $\operatorname{Frac}\left(K \llbracket t_{1} \rrbracket_{0}\right)$ with respect to the 1 -Gauss norm.

By applying the induction hypothesis to $\widetilde{M}=M \otimes_{R} \widetilde{R}$, the multiset $A$ is equal to

$$
\begin{array}{r}
A^{\prime}=\left\{\left(\mathrm{v}_{s}\left(\vartheta^{\prime}\right),-b_{2}, \ldots,-b_{n}, \overline{t_{1}^{-v_{s}\left(\vartheta^{\prime}\right)} \vartheta^{\prime}}\right) \mid\left(-b_{2}, \ldots,-b_{n}\right) \in \mathbb{Q}^{n-1},\right. \\
\left.t_{2}^{-b_{2}} \cdots t_{n}^{-b_{n}} \vartheta^{\prime} \in \mathscr{I} \Theta\left(M \otimes F_{\eta_{I}}\right)\right\},
\end{array}
$$

for any $\left(r_{2}, \ldots, r_{n}\right) \in \widetilde{C}^{\prime}$, where $\mathrm{v}_{s}$ is the valuation on

$$
\bigoplus_{i \in I} \kappa_{\widetilde{K}^{\text {alg }}}^{(s)} \frac{d t_{i}}{t_{i}} \oplus \bigoplus_{j \in J} \kappa_{\widetilde{K}^{\text {alg }}}^{(s)} \frac{d u_{j}}{u_{j}}
$$

as in Notation 4.2.3, and $\overline{t_{1}^{-v_{s}\left(\vartheta^{\prime}\right)} \vartheta^{\prime}}$ is the reduction of $t_{1}^{-v_{s}\left(\vartheta^{\prime}\right)} \vartheta^{\prime}$ in

$$
\bigoplus_{i \in I} \kappa_{K^{\mathrm{alg}}}^{(s)} \frac{d t_{i}}{t_{i}} \oplus \bigoplus_{j \in J} \kappa_{K^{\mathrm{alg}}}^{(s)} \frac{d u_{j}}{u_{j}} .
$$

It suffices to identify the multiset $A^{\prime}$ with $B$. When $r_{I} \in \mathbb{Q}^{n} \cap C^{\prime}$, this follows from applying Corollary 4.2.7 to the line which passes through the point $r_{I}$ and is parallel to the $t_{1}$-axis. In particular, this says that for any $\vartheta^{\prime}$ above,

$$
\overline{t_{1}^{-v_{s}\left(\vartheta^{\prime}\right)} \vartheta^{\prime}} \text { is the same as } \vartheta \text {. }
$$


When $r_{I}$ is not rational, the same statement follows from the "continuity" result in Theorem 4.3.6(c).

Remark 4.3.9. One can also describe the intrinsic radii of $M_{b_{I}, \vartheta}$ at the point $\left(r_{I}\right) \in C^{\prime}$ with $r_{1}=\cdots=r_{l}=0$ for some $l \in\{1, \ldots, d-1\}$. We leave this as an exercise for interested readers.

Next we consider the situation for solvable differential modules.

Definition 4.3.10. Let $C=\left\{\left(x_{I}\right) \subset \mathbb{R}^{n}: x_{I} \geq 0, x_{1}+\cdots+x_{n}=1\right\}$. For $[\alpha, \beta] \in(0,1)$, we put $S_{[\alpha, \beta]}=\left\{\rho^{C}: \rho \in[\alpha, \beta]\right\}$ and $R_{[\alpha, \beta]}=K \llbracket S_{[\alpha, \beta]} \rrbracket_{0}$. For $\alpha \in(0,1)$, we put $R_{\alpha}=\bigcap_{\beta \in(\alpha, 1)} R_{[\alpha, \beta]}$.

Fix $\alpha \in(0,1)$. Let $M$ be a differential module over $R_{\alpha}$. Assume that $M$ is solvable, that is, for each $x_{I} \in C$, we have $f_{1}\left(M, \rho^{x_{I}}\right) \rightarrow 0$ as $\rho \rightarrow 1^{-}$.

By Theorem 1.6.2, for $x_{I} \in C$, there exists $b_{1}\left(M, x_{I}\right), \ldots, b_{d}\left(M, x_{I}\right)$ such that $f_{l}\left(M,-x_{I} \log \rho\right)=\rho^{b_{l}\left(M, x_{I}\right)}$ when $\rho \rightarrow 1^{-}$, for $l=1, \ldots, d$. Put

$$
B_{l}\left(M, x_{I}\right)=b_{1}\left(M, x_{I}\right)+\cdots+b_{l}\left(M, x_{I}\right)
$$

for $l=1, \ldots, d$.

Proposition 4.3.11. Keep the notation as above. Then the functions $d ! B_{l}\left(M, x_{I}\right)$ and $B_{d}\left(M, x_{I}\right)$ are integral polyhedral functions.

Proof. See [Kedlaya 2011, Theorem 3.3.3]. The proposition also follows from Theorem 4.3.6(a).

Construction 4.3.12. Keep the notation as above.

Let $\underline{x}=(0, \ldots, 1) \in C$ be the point. Let $\mathfrak{F}$ be the completion of the fraction field of $\mathrm{O}_{K}\left(\left(t_{1}\right)\right) \cdots\left(\left(t_{n-1}\right)\right)$; it is a higher dimensional local field. We have a natural embedding $R_{\alpha} \hookrightarrow \mathfrak{F}\left\{\left\{\eta / t_{n}, t_{n}\right\}\right\}=\widetilde{\mathfrak{F}}_{\eta}$, if $\eta \in(\alpha, 1)$. This means to restrict the picture to the line $(0, \ldots, 0, \rho)$ for $\rho \in(\eta, 1)$. We assume that $M \otimes \widetilde{\mathfrak{F}}_{\eta}$ has pure-log break $b$.

Recall that, as in Situation 4.3.7, we have a valuation

$$
\boldsymbol{v}: \bigoplus_{i \in I} \kappa_{\mathfrak{F}^{\mathrm{alg}}} \frac{d t_{i}}{t_{i}} \oplus \bigoplus_{j \in J} \kappa_{\mathfrak{F}^{\mathrm{alg}}} \frac{d u_{j}}{u_{j}} \rightarrow \mathbb{Q}^{n}
$$

Proposition 4.3.13. Keep the notation as above. The following two multisets of $(n-1)$-tuples are the same.

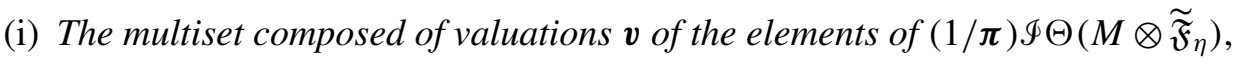
where $\pi$ is a Dwork pi.

(ii) The multiset of slopes of $b_{l}\left(M, x_{I}\right)$, for $l=1, \ldots, d$, on a RP subset of $C$ which is adjacent to the cells $\left\{t_{1}=\cdots=t_{i}=0, t_{i+1}+\cdots+t_{n}=1\right\}$ for all $i=1, \ldots, n$.

Proof. It follows from Theorem 4.3.8. 
Remark 4.3.14. One may interpret the above proposition geometrically, as in [Kedlaya 2011]. We will come back to this discussion in a future work.

\section{Acknowledgments}

I would like to thank my advisor, Kiran Kedlaya, for generating some of crucial ideas, for helpful discussions, and for spending hours reviewing early drafts. I thank Ivan Fesenko for suggesting the application to higher local fields. I have benefited from discussions with Takeshi Saito. I thank Ahmed Abbes for inviting me to the conference "Journées de Géométrie Arithmétique de Rennes", which has benefited me a lot. I am also grateful for Matthew Morrow and Sandeep Varma for their help with the presentation of this paper.

\section{References}

[Abbes and Saito 2002] A. Abbes and T. Saito, "Ramification of local fields with imperfect residue fields", Amer. J. Math. 124:5 (2002), 879-920. MR 2003m:11196 Zbl 1084.11064

[Abbes and Saito 2003] A. Abbes and T. Saito, "Ramification of local fields with imperfect residue fields, II”, pp. 5-72 in Kazuya Kato's fiftieth birthday, edited by S. Bloch et al., Doc. Math. 3, Documenta Mathematica, Bielefeld, 2003. MR 2005g:11231 Zbl 1127.11349

[Abbes and Saito 2009] A. Abbes and T. Saito, "Analyse micro-locale $l$-adique en caractéristique $p>0$ : le cas d'un trait", Publ. Res. Inst. Math. Sci. 45:1 (2009), 25-74. MR 2009m:11197 Zbl 1225.11151

[Berkovich 1990] V. G. Berkovich, Spectral theory and analytic geometry over non-Archimedean fields, Mathematical Surveys and Monographs 33, American Mathematical Society, Providence, RI, 1990. MR 91k:32038 Zbl 0715.14013

[Berthelot 1996] P. Berthelot, "Cohomologie rigide et cohomologie rigide à support propre, première partie”, preprint IRMAR 96-03, Université de Rennes, 1996, available at http://perso.univ-rennes1.fr/ pierre.berthelot/publis/Cohomologie_Rigide_I.pdf.

[Chiarellotto and Pulita 2009] B. Chiarellotto and A. Pulita, "Arithmetic and differential Swan conductors of rank one representations with finite local monodromy", Amer. J. Math. 131:6 (2009), 1743-1794. MR 2011a:14036 Zbl 1198.12004

[Dwork et al. 1994] B. Dwork, G. Gerotto, and F. J. Sullivan, An introduction to G-functions, Annals of Mathematics Studies 133, Princeton University Press, 1994. MR 96c:12009 Zbl 0830.12004

[Kato 1989] K. Kato, "Swan conductors for characters of degree one in the imperfect residue field case", pp. 101-131 in Algebraic K-theory and algebraic number theory (Honolulu, HI, 1987), edited by M. R. Stein and R. K. Dennis, Contemp. Math. 83, American Mathematical Society, Providence, RI, 1989. MR 90g:11164 Zbl 0716.12006

[Kedlaya 2005] K. S. Kedlaya, "Local monodromy of p-adic differential equations: An overview", Int. J. Number Theory 1:1 (2005), 109-154. Correction in. MR 2006g:12013 Zbl 1107.12005

[Kedlaya 2007] K. S. Kedlaya, "Swan conductors for $p$-adic differential modules, I: A local construction”, Algebra Number Theory 1:3 (2007), 269-300. MR 2009b:11205 Zbl 1184.11051

[Kedlaya 2009] K. S. Kedlaya, "Semistable reduction for overconvergent $F$-isocrystals, III: Local semistable reduction at monomial valuations", Compos. Math. 145:1 (2009), 143-172. MR 2009k: 14040 Zbl 1184.14031 
[Kedlaya 2010] K. S. Kedlaya, p-adic differential equations, Cambridge Studies in Advanced Mathematics 125, Cambridge University Press, 2010. MR 2011m:12016 Zbl 1213.12009

[Kedlaya 2011] K. S. Kedlaya, "Swan conductors for $p$-adic differential modules, II: Global variation”, J. Inst. Math. Jussieu 10:1 (2011), 191-224. MR 2012d:11231 Zbl 05838439

[Kedlaya and Xiao 2010] K. S. Kedlaya and L. Xiao, "Differential modules on p-adic polyannuli", J. Inst. Math. Jussieu 9:1 (2010), 155-201. MR 2011j:14055 Zbl 1195.12008

[Ore 1933] O. Ore, "Theory of non-commutative polynomials", Ann. of Math. (2) 34:3 (1933), 480-508. MR 1503119 Zbl 0007.15101

[Saito 2009] T. Saito, "Wild ramification and the characteristic cycle of an $l$-adic sheaf", J. Inst. Math. Jussieu 8:4 (2009), 769-829. MR 2011e:14039 Zbl 1177.14044

[Xiao 2009] L. Xiao, Nonarchimedean differential modules and ramification theory, Ph.D. thesis, Massachusetts Inst. of Technology, 2009, available at http://hdl.handle.net/1721.1/50596. MR 2717735

[Xiao 2010] L. Xiao, "On ramification filtrations and $p$-adic differential modules, I: The equal characteristic case”, Algebra Number Theory 4:8 (2010), 969-1027. MR 2832631 Zbl 1225.11152

Communicated by Brian Conrad

Received 2010-06-10 Revised 2011-12-19 Accepted 2012-01-17

Ixiao@math.uchicago.edu Department of Mathematics, University of Chicago, 5734 S. University Ave, Chicago, IL 60637, United States 


\section{Algebra \& Number Theory}

msp.berkeley.edu/ant

\section{EDITORS}

MANAGING EDITOR

Bjorn Poonen

Massachusetts Institute of Technology

Cambridge, USA

\author{
EDITORIAL BOARD CHAIR \\ David Eisenbud \\ University of California \\ Berkeley, USA
}

\section{BOARD OF EDITORS}

Georgia Benkart

Dave Benson

Richard E. Borcherds

John H. Coates

J-L. Colliot-Thélène

Brian D. Conrad

Hélène Esnault

Hubert Flenner

Edward Frenkel

Andrew Granville

Joseph Gubeladze

Ehud Hrushovski

Craig Huneke

Mikhail Kapranov

Yujiro Kawamata

János Kollár

Yuri Manin

Barry Mazur

Philippe Michel
University of Wisconsin, Madison, USA

University of Aberdeen, Scotland

University of California, Berkeley, USA

University of Cambridge, UK

CNRS, Université Paris-Sud, France

University of Michigan, USA

Freie Universität Berlin, Germany

Ruhr-Universität, Germany

University of California, Berkeley, USA

Université de Montréal, Canada

San Francisco State University, USA

Hebrew University, Israel

University of Virginia, USA

Yale University, USA

University of Tokyo, Japan

Princeton University, USA

Northwestern University, USA

Harvard University, USA

École Polytechnique Fédérale de Lausanne
Susan Montgomery

Shigefumi Mori

Raman Parimala

Jonathan Pila

Victor Reiner

Karl Rubin

Peter Sarnak

Joseph H. Silverman

Michael Singer

Vasudevan Srinivas

J. Toby Stafford

Bernd Sturmfels

Richard Taylor

Ravi Vakil

Michel van den Bergh

Marie-France Vignéras

Kei-Ichi Watanabe

Andrei Zelevinsky

Efim Zelmanov
University of Southern California, USA

RIMS, Kyoto University, Japan

Emory University, USA

University of Oxford, UK

University of Minnesota, USA

University of California, Irvine, USA

Princeton University, USA

Brown University, USA

North Carolina State University, USA

Tata Inst. of Fund. Research, India

University of Michigan, USA

University of California, Berkeley, USA

Harvard University, USA

Stanford University, USA

Hasselt University, Belgium

Université Paris VII, France

Nihon University, Japan

Northeastern University, USA

University of California, San Diego, USA

\section{PRODUCTION}

production@msp.org

Silvio Levy, Scientific Editor

See inside back cover or www.jant.org for submission instructions.

The subscription price for 2012 is US \$175/year for the electronic version, and \$275/year ( $\$ 40$ shipping outside the US) for print and electronic. Subscriptions, requests for back issues from the last three years and changes of subscribers address should be sent to Mathematical Sciences Publishers, Department of Mathematics, University of California, Berkeley, CA 94720-3840, USA.

Algebra \& Number Theory (ISSN 1937-0652) at Mathematical Sciences Publishers, Department of Mathematics, University of California, Berkeley, CA 94720-3840 is published continuously online. Periodical rate postage paid at Berkeley, CA 94704, and additional mailing offices.

ANT peer review and production are managed by EditFLOW ${ }^{\circledR}$ from Mathematical Sciences Publishers.

PUBLISHED BY

mathematical sciences publishers

http://msp.org/

A NON-PROFIT CORPORATION

Typeset in IATEX

Copyright ( 2012 by Mathematical Sciences Publishers 


\section{Algebra \& Number Theory}

Volume $6 \quad$ No. $8 \quad 2012$

On the refined ramification filtrations in the equal characteristic case

LIANG XIAO

On common values of $\phi(n)$ and $\sigma(m)$, II

1669

KEVIN FORD and PAUL POLLACK

Galois representations associated with unitary groups over $\mathbb{Q}$

CHRISTOPHER SKINNER

Abelian varieties and Weil representations

SUG WOO SHIN

Small-dimensional projective representations of symmetric and alternating groups

Alexander S. KleshcheV and Pham HuU TieP

Secant varieties of Segre-Veronese varieties

1817

Claudiu Raicu 